\title{
Modelling the corona of stars more active than the Sun using 3D MHD simulations
}

\author{
Dissertation \\ zur Erlangung des mathematisch-naturwissenschaftlichen Doktorgrades \\ "Doctor rerum naturalium" \\ der Georg-August-Universität Göttingen \\ im Promotionsstudiengang Physik \\ der Georg-August University School of Science (GAUSS)
}

vorgelegt von

Juxhin Zhuleku

aus Tirana, Albanien

Göttingen, 2021 
Betreuungsausschuss

Prof. Dr. Hardi Peter

Max-Planck-Institut für Sonnensystemforschung, Göttingen, Germany

Dr. Robert Cameron

Max-Planck-Institut für Sonnensystemforschung, Göttingen, Germany

Prof. Dr. Andreas Tilgner

Georg-August-Universität, Göttingen, Germany

Mitglieder der Prüfungskommision

Referent: Prof. Dr. Hardi Peter

Max-Planck-Institut für Sonnensystemforschung, Göttingen, Germany

Korreferent: Prof. Dr. Andreas Tilgner

Georg-August-Universität, Göttingen, Germany

Weitere Mitglieder der Prüfungskommission:

Prof. Dr. Stefan Dreizler

Institut für Astrophysik, Georg-August-Universität, Göttingen, Germany

Prof. Dr. Wolfram Kollatschny

Institut für Astrophysik, Georg-August-Universität, Göttingen, Germany

Dr. Petri Käpylä

Institut für Astrophysik, Georg-August-Universität, Göttingen, Germany

Dr. Jörn Warnecke

Max-Planck-Institut für Sonnensystemforschung, Göttingen, Germany

Tag der mündlichen Prüfung: 19.02.2021 


\section{Bibliografische Information der Deutschen Nationalbibliothek}

Die Deutsche Nationalbibliothek verzeichnet diese Publikation in der Deutschen Nationalbibliografie; detaillierte bibliografische Daten sind im Internet über http: //dnb . d-nb . de abrufbar.

(C) Juxhin Zhuleku

(c) This work is distributed under a
Creative Commons Attribution 4.0 License

Printed in Germany 



\section{Contents}

$\begin{array}{ll}\text { Zusammenfassung } & 9\end{array}$

$\begin{array}{ll}\text { Summary } & 11\end{array}$

1 Introduction $\quad 13$

1.1 Solar corona . . . . . . . . . . . . . . . . . . . . . . . . . . . . . . . 13

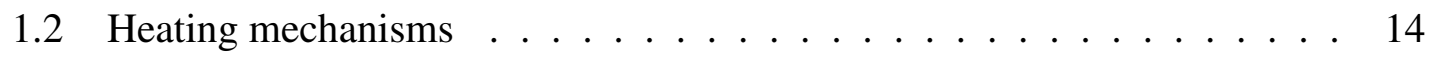

1.2 .1 AC heating ...................... 15

1.2 .2 DC heating . . . . . . . . . . . . . . 16

1.3 Solar-stellar connection . . . . . . . . . . . . . . . . . . . 17

1.3.1 Rotation-activity relation . . . . . . . . . . . . . 18

1.3.2 Observed X-ray activity to surface magnetic flux relation . . . . . 19

1.4 Motivation and aim of the present work . . . . . . . . . . 20

2 Theoretical framework 23

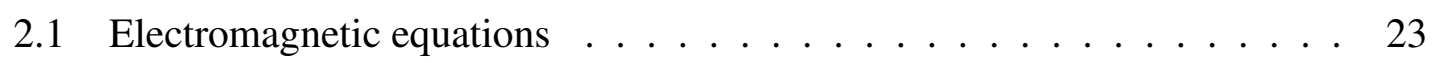

2.1 .1 Maxwell's equations . . . . . . . . . . . . 23

2.1 .2 Induction equation $\ldots \ldots \ldots \ldots . \ldots \ldots$

2.1 .3 Poynting theorem . . . . . . . . . . . . . . . . . . 25

2.2 Fluid equations . . . . . . . . . . . . . . . . . . 26

2.2 .1 Continuity equation . . . . . . . . . . . . . 26

2.2 .2 Momentum equation . . . . . . . . . . . . . 26

2.2 .3 Equation of state . . . . . . . . . . . . . . . . . 27

2.2 .4 Energy equation . . . . . . . . . . . . . . . 27

2.3 Basic characteristics of coronal loops . . . . . . . . . . . . . . . 30

2.3.1 One dimensional coronal loop models . . . . . . . . . . . . . 31

2.3.2 Three dimensional numerical models of coronal loops above ac-

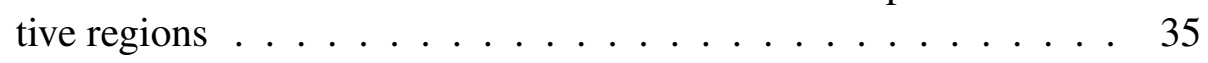

2.3.3 Stellar coronal loops . . . . . . . . . . . . 36

3 Stellar coronal X-ray emission and surface magnetic flux 39

3.1 Introduction . . . . . . . . . . . . . . . . . . . . . . 39

3.2 Temperature dependence of X-ray radiation . . . . . . . . . . . . . . . . 41

3.3 Magnetic field and heating of coronal plasma . . . . . . . . . . . . . . . 43

3.4 Scaling laws: coronal emission vs. magnetic flux . . . . . . . . . . . . 45

3.5 Discussion . . . . . . . . . . . . . . . . . . 47 
3.5.1 Discriminating heating mechanisms . . . . . . . . . . . . 47

3.5.2 Magnetic flux and area coverage . . . . . . . . . . . . . 48

3.5.3 Spatial structure of the magnetic field . . . . . . . . . . . . . . 49

3.5.4 X-ray emission for rapid rotators . . . . . . . . . . . . . . . . 50

3.6 Conclusions . . . . . . . . . . . . . . . . . . 50

4 Model setup 53

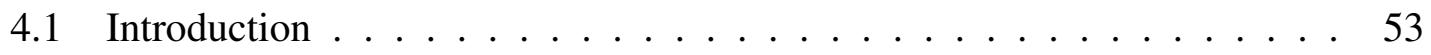

4.2 Numerical model . . . . . . . . . . . . . . . . . . . . 54

4.2 .1 Equations . . . . . . . . . . . . . . . . 54

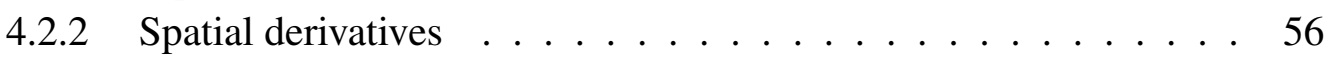

4.2.3 Time step constraints . . . . . . . . . . . . . . . 56

4.3 Initial conditions . . . . . . . . . . . . . . . . . . 60

4.4 Potential field extrapolation . . . . . . . . . . . . . . . . . . . . . . 60

4.5 Boundary conditions . . . . . . . . . . . . . . . 62

4.5.1 Photospheric driver . . . . . . . . . . . . . . . 62

4.5.2 Photospheric magnetic field update . . . . . . . . . . . 63

4.6 Verification of the numerical model . . . . . . . . . . . . . . . . 64

4.6.1 Ohmic heating . . . . . . . . . . . . . . . . 64

4.6.2 Coronal temperature and density . . . . . . . . . . . . 65

4.6 .3 Coronal energy flux . . . . . . . . . . . . . . . . 66

4.6 .4 Nanoflares . . . . . . . . . . . . . . . . . 68

4.6.5 Synthetic coronal emission . . . . . . . . . . . . 70

5 Stellar X-rays and magnetic activity in 3D MHD coronal models 75

5.1 Introduction . . . . . . . . . . . . . . . . . . . 75

5.2 Analytical scaling relations . . . . . . . . . . . . . . . . 76

5.3 Numerical model setup . . . . . . . . . . . . . . . . . 78

5.3 .1 Basic equations . . . . . . . . . . . . . . 78

5.3.2 Initial and boundary conditions . . . . . . . . . . . 79

5.4 Numerical experiments . . . . . . . . . . . . . . . . . . 80

5.4 .1 Setup . . . . . . . . . . . . . . . . 80

5.4.2 Synthesized emission: X-rays and EUV . . . . . . . . . . . . . . 81

5.4 .3 Horizontal averages . . . . . . . . . . . . . . . . . . 82

5.4 .4 Temporal evolution . . . . . . . . . . . . . . 85

5.5 Scaling relations in numerical experiments . . . . . . . . . . . . 87

5.6 Discussion . . . . . . . . . . . . . . . . . . . 89

5.6.1 Energy input into the corona . . . . . . . . . . . . . . . 89

5.6.2 RTV scaling laws compared to numerical experiments . . . . . 90

5.6.3 Relation of X-ray emission to surface magnetic flux . . . . . . . . 91

5.6.4 Analytical model for scaling of X-ray emission . . . . . . . . . 93

5.6.5 X-ray and EUV emission versus coronal energy input . . . . . . . 93

5.6.6 Relating the X-rays the EUV emission . . . . . . . . . . . . . . . 94

5.7 Conclusions . . . . . . . . . . . . . . . . . . . . 95 
6 Influence of the size of the active region on the coronal X-ray emission 97

6.1 Introduction . . . . . . . . . . . . . . . . . . . . 97

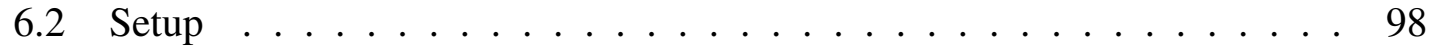

6.3 Results . . . . . . . . . . . . . . . . . . . . . 99

6.3.1 Synthetic coronal emission and temporal evolution . . . . . . . . 99

6.3.2 Coronal temperature and density . . . . . . . . . . . . 102

6.3.3 Coronal heating . . . . . . . . . . . . . . . 103

6.3.4 Exponential decay of the magnetic field . . . . . . . . . . . . 105

6.3.5 Coronal heating problem revisited . . . . . . . . . . . . . 107

6.3.6 Coronal X-ray emission . . . . . . . . . . . . . . 108

6.4 Conclusion . . . . . . . . . . . . . . . . . 110

7 Conclusion and Discussion $\quad 111$

$\begin{array}{ll}\text { Bibliography } & 115\end{array}$

$\begin{array}{lr}\text { Scientific contributions } & 123\end{array}$

$\begin{array}{lr}\text { Acknowledgements } & 125\end{array}$ 



\section{Zusammenfassung}

Kühle Sterne wie unsere Sonne sind von Koronae mit Temperaturen bis zu mehreren Millionen Kelvin umgeben. Auch nach jahrzehntelanger Forschung gibt es keine eindeutige Erklärung dafür, warum die Koronae von Sternen um mehrere Größenordnungen heißer sind als ihre Oberflächen. Aufgrund der hohen Temperatur weisen die solaren und stellaren Koronae eine hohe Röntgenemission auf. Koronae von Sternen, die aktiver sind als die Sonne, können im Allgemeinen sogar eine 1000-mal stärkere Röntgenemission aufweisen als die Sonnenkorona.

Die koronale Röntgenaktivität der Sonne und anderer Sterne wird durch das Oberflächenmagnetfeld bestimmt. Zahlreiche Beobachtungsstudien haben gezeigt, dass die Beziehung zwischen der koronalen Röntgenleuchtkraft $L_{X}$ und dem magnetischen Fluss auf der Oberfläche $\Phi$ der Sonne und anderer Sterne einem Potenzgesetzbeziehun, $L_{\mathrm{X}} \propto$ $\Phi^{m}$, folgt. Je nach Studie ist $1 \leq m<3$. Bis jetzt gibt es keine eindeutige Erklärung dafür, warum es eine Potenzgesetzabhängigkeit zwischen $L_{X}$ und $\Phi$ gibt und auch nicht dafür, warum $m$ von einer Studie zur anderen variiert. In dieser Arbeit versuchen wir, diese Potenzgesetzbeziehung durch ein einfaches analytisches Modell und durch 3Dmagnetohydrodynamische (MHD) Modelle der solaren und stellaren Koronae quantitativ zu erklären.

Unser analytisches Modell basiert auf einer Kombination des Rosner, Tucker \& Vaiana (RTV) Skalierungsgesetzes (Rosner et al. 1978), koronalen Heizmechanismen, zum Beispiel Nanoflares, und der Temperaturabhängigkeit für verschiedene Instrumente. Daraus ergibt sich ein einfacher analytischer Potenzgesetzausdruck, der die Potenzgesetzbeziehung zwischen der Röntgenhelligkeit $L_{\mathrm{X}}$ und dem magnetischen Fluss auf der Oberfläche $\Phi$ erklären kann. Der Potenzgesetzindex $m$ liegt im Bereich von 0.8 bis 1.6, was mit dem von Beobachtungsstudien berichteten Bereich von $m$ übereinstimmt. Darüber hinaus finden wir, dass die Empfindlichkeit jedes einzelnen Instruments in einem bestimmten Temperaturbereich einen signifikanten Einfluss auf den Potenzgesetzindex $m$ haben kann. Dies wurde bei allen Beobachtungsstudien übersehen.

Um die $L_{\mathrm{X}} \propto \Phi^{m}$-Beziehung in einer komplexeren Umgebung weiter zu untersuchen, verwenden wir numerische 3D-MHD-Modelle, um den Teil der Korona über einer aktiven Region zu simulieren. Wir verwenden das von Bingert (2009) entwickelte solare Koronamodell, das einige der Schlüsselaspekte der koronalen Strukturen erfolgreich reproduziert hat. Eine heiße und dynamische Korona mit Temperaturen von $1 \mathrm{MK}$ und mehr wird dabei selbstkonsistent erzeugt. Wir untersuchen, wie sich die koronale Röntgenleuchtkraft mit der Magnetfeldstärke und der Größe der darunter liegenden aktiven Region ändert.

Zunächst erhöhen wir die Stärke des vertikalen Oberflächenmagnetfeldes um einen konstanten Faktor, während wir die Größe der aktiven Region konstant halten. Mit diesem 
Ansatz erreichen wir Werte des Oberflächenmagnetfeldes von bis zu 20 kG. Dieser Wert des Magnetfelds ist für die Sonne extrem hoch, aber es wird vermutet, dass er bei aktiveren Sternen üblich ist. Die von unserem Modell erhaltene koronale Temperatur und Dichte sind in guter Übereinstimmung mit den RTV-Skalierungsgesetzen. Außerdem steigt die aus unserem Modell synthetisierte koronale Röntgenleuchtkraft $L_{X}$ mit dem Oberflächenmagnetfluss $\Phi$, was mit anderen Studien übereinstimmt. Wir finden, dass diese Beziehung ein Potenzgesetz $L_{\mathrm{X}} \propto \Phi^{m}$ mit dem Potenzgesetzindex $m=3.4$ folgt. Zweitens, erhöhen wir den gesamten magnetischen Oberflächenfluss, indem wir die Fläche der aktiven Region vergrößern, während wir die Stärke des Oberflächenmagnetfeldes konstant halten. Wir sehen einen Anstieg der koronalen Temperatur, obwohl der Anstieg nicht so stark ist wie im ersten Ansatz. Die synthetische Röntgenemission steigt mit dem Oberflächenmagnetfluss, in Übereinstimmung mit dem vorherigen Ansatz. In diesem Fall ist der Index mit $m \simeq 2.2$ jedoch weniger steil als beim ersten Ansatz.

Insgesamt liefern unsere Ergebnisse neue Einblicke in die $L_{X} \propto \Phi^{m}$-Beziehung. Die Empfindlichkeit der einzelnen Instrumente in einem bestimmten Temperaturbereich kann die in den Beobachtungen gefundene Differenz von $m$ erklären. Darüber hinaus können unser analytisches Modell und numerische Experimente eine Erklärung dafür liefern, warum die Abhängigkeit der Röntgenleuchtkraft $L_{X}$ und des magnetischen Oberflächenflusses ein Potenzgesetz ist. 


\section{Summary}

Cool stars like our Sun are surrounded by coronae with temperatures up to several million Kelvin. After decades of research, there has been no definite explanation as to why the coronae of stars are several orders of magnitude hotter than their surfaces. Because of the high temperature, the solar and stellar coronae exhibit high X-ray emission. Coronae of stars more active than the Sun can generally appear to have even 1000 times stronger $\mathrm{X}$-ray emission than the solar corona.

The coronal X-ray activity of the Sun and other stars is governed by the surface magnetic field. The relationship between the coronal X-ray luminosity $L_{X}$ and the surface magnetic flux $\Phi$ of the Sun and other stars has been shown to follow a power-law relation, $L_{\mathrm{X}} \propto \Phi^{m}$, by numerous observational studies. Depending on the study, $1 \leq m<3$. Until now there is no clear explanation of why there is a power-law dependence between $L_{X}$ and $\Phi$ and also why $m$ is found to differ from one study to another. In this thesis, we aim to explain this power-law relationship quantitatively through a simple analytical model and through 3D magnetohydrodynamic (MHD) models of the solar and stellar coronae.

Our analytical model is based on a combination of the Rosner, Tucker \& Vaiana (RTV) scaling laws (Rosner et al. 1978), coronal heating mechanisms, for example nano flares, and the temperature response for different instruments. This results in a simple analytical power-law expression, that can explain the power-law relation between the X-ray luminosity $L_{X}$ and the surface magnetic flux $\Phi$. The power-law index $m$ is found to be in the range from 0.8 to 1.6 which is in agreement with the range of $m$ reported by observational studies. Furthermore, we also find that the sensitivity of each individual instrument at a specific temperature range can have a significant influence on the power-law index $m$. This has been overlooked for all observational studies.

To further investigate the $L_{\mathrm{X}} \propto \Phi^{m}$ relationship in a more complex environment, we use 3D MHD numerical models to simulate the part of the corona above an active region. We use the solar coronal model developed by Bingert (2009), that has successfully reproduced some of the key aspects of coronal structures. A hot and dynamic corona with temperatures of $1 \mathrm{MK}$ and more is self-consistently created. We investigate how the coronal X-ray emission changes with the magnetic field strength and the size of the underlying active region.

Firstly, we increase the strength of the vertical surface magnetic field by a constant factor while keeping the size of the active region constant. With this approach, we reach values of the surface magnetic field up to $20 \mathrm{kG}$. This value of the magnetic field is extremely high for the Sun but it is speculated to be common in more active stars. The coronal temperature and density obtained by our model are in good agreement with the RTV scaling laws. Furthermore, the coronal X-ray luminosity $L_{\mathrm{X}}$ synthesized from our model increases with the surface magnetic flux $\Phi$, which is consistent with other studies. 
We find this relation to be a power-law $L_{X} \propto \Phi^{m}$ with the power-law index $m=3.4$. Secondly, we increase the overall surface magnetic flux by increasing the size of the active region while keeping the strength of the surface magnetic field constant. We see an increase in the coronal temperature, although the increase is not as strong as in the first approach. The synthetic X-ray emission increases with the surface magnetic flux, in agreement with the previous approach. In this case, however, the index $m \simeq 2.2$ is found to be less steep than for the first approach.

Overall, our results provide new insight into the $L_{X} \propto \Phi^{m}$ relationship. The sensitivity of each instrument at a specific temperature range can explain the difference of $m$ found in observations. In addition, our analytical model and numerical experiments can provide an explanation of why the dependence of the X-ray luminosity $L_{X}$ and the surface magnetic flux is a power-law. 


\section{Introduction}

\subsection{Solar corona}

The solar corona is regarded as the outer atmosphere surrounding the Sun. Its faint optical emission compared to the bright photosphere makes it impossible to observe with naked eye. Since ancient times, people could observe the solar corona only during solar eclipses as a faint halo surrounding the Moon (see Fig. 1.1). Hence, it was believed to be part of the lunar atmosphere. The fact that the corona was part of the solar atmosphere was proven only in the late 1800 s.

Based on the first spectrographic observations, it was believed that the emission lines observed from the solar corona were due to a new element, which was named coronium. It was only after the 1930s when scientists realized that the emission was originating from highly ionized elements such as iron. The presence of highly ionized elements in the solar corona revealed its mystery. The solar corona has a mean temperature of $10^{6} \mathrm{~K}$, which is more than 100 times higher than the surface temperature (Grotrian 1939; Edlén 1943).

After decades of studying the Sun, there is now a clear picture of how the solar atmosphere is stratified reaching, from a photosphere of $6000 \mathrm{~K}$ to a corona of $10^{6} \mathrm{~K}$. The solar atmosphere is filled with plasma and based on 1D models (see e.g. Vernazza et al. 1981), it can be roughly divided into four layers (see Fig. 1.2). The first layer is defined as the photosphere. The photosphere is considered to be the "surface" of the Sun, and most of the phenomena observed in the corona or transition region are associated with structures located in that region, such as active regions or faculae. The second layer is the chromosphere. It is a cool, and dense layer with a temperature of around $10^{4} \mathrm{~K}$. Its nature is still not fully understood, however, it is speculated that magnetohydrodynamic (MHD) waves might be responsible for the temperature increase (see the review from Mathioudakis et al. 2013). The region between the chromosphere and the corona is a thin layer of a few hundred $\mathrm{km}$ called the transition region. In that region, a steep increase in temperature and a decrease in plasma density is observed. The last layer is the low density and extremely high-temperature corona that can extend to very high altitude.

The solar corona has a very faint optical emission. The low density and high-temperature coronal plasma suggest a thermal emission at short wavelengths, for example, the extremeultraviolet (EUV) wavelength regime. The solar photosphere emits like a black body with a temperature of $6000 \mathrm{~K}$. Because of the high temperature, the corona appears brighter at short wavelengths, such as the EUV or X-ray regime, compared to the photosphere. Hence, the EUV and X-ray wavelength range is widely used for coronal diagnostics. Since the Earth's atmosphere blocks the EUV and X-ray emission, space-based instruments have to be used for the observations. These instruments, such as Solar and He- 


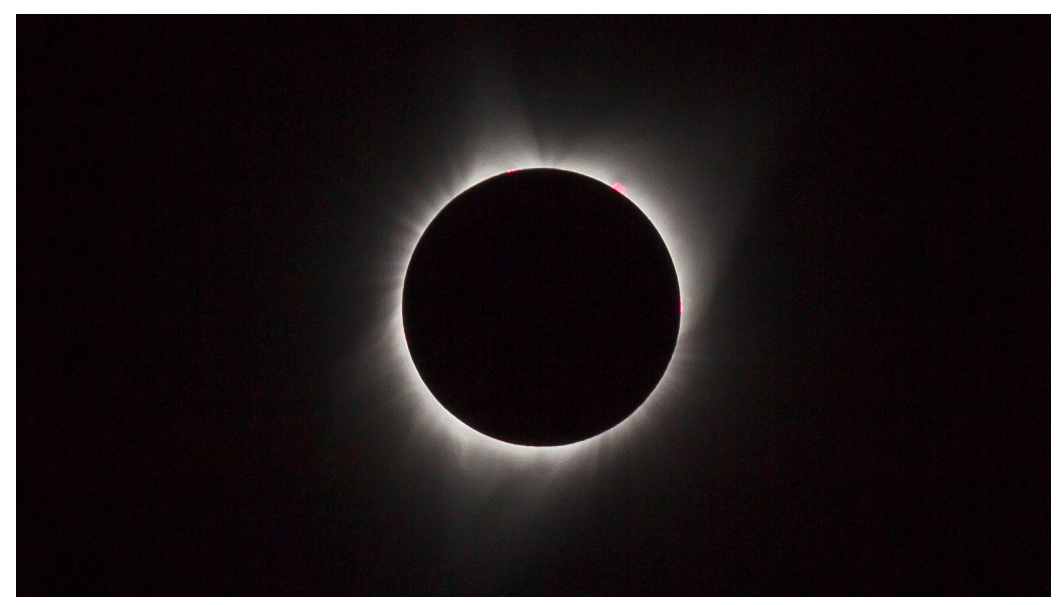

Figure 1.1: Solar eclipse seen from Portland, USA, on August 21, 2017. Courtesy: Pradeep L. Chitta

liospheric Observatory (SOHO; Domingo et al. 1995), Transition Region and Coronal Explorer (TRACE; Handy et al. 1999), and others can observe the solar corona in different wavelengths such as the $171 \AA$ channel of Atmospheric Imaging Assembly (AIA; Lemen et al. 2012) onboard Solar Dynamic Observatory (SDO; Pesnell et al. 2012) (see Fig. 1.3).

The first observations of the solar corona were obtained by the space mission of Skylab in early 70s (MacQueen et al. 1974). Some of the features that can be distinguished are, first of all, coronal holes, where plasma escapes through the open magnetic fieldlines. Corona holes are speculated to have a connection to the solar wind. Furthermore, there are also the bright coronal loops, that connect regions of opposite magnetic polarity (see Fig. 1.3), and they are considered to significantly contribute to coronal heating. The plasma in the coronal loops is confined, and it can move only along the magnetic fieldlines. More details follow in Chap. 2. An additional feature observed in the solar corona are the X-ray bright points scattered throughout the whole disk with an average size of roughly $20 \mathrm{Mm}$ and a typical lifetime of 8 hours (see the book from Priest (2014) for more details).

The activity and the appearance of the individual features of the solar corona strongly depend on the solar cycle. It is observed that during the solar maximum, the solar corona shows enhanced EUV emission and it is structured. At the same time, most of the solar surface is covered with magnetic structures, such as active regions, as it is illustrated in Fig. 1.3. During the solar minimum, the solar corona shows a diffused emission and, only a few active regions are present. That indicates a connection between the surface magnetic field (active regions) and coronal emission. The mechanism responsible for converting magnetic energy to thermal energy is still under debate.

\subsection{Heating mechanisms}

For more than 80 years, the physical process that leads to a high-temperature corona $\left(T \sim 10^{6} \mathrm{~K}\right)$ was poorly understood. The second law of thermodynamics suggests that 


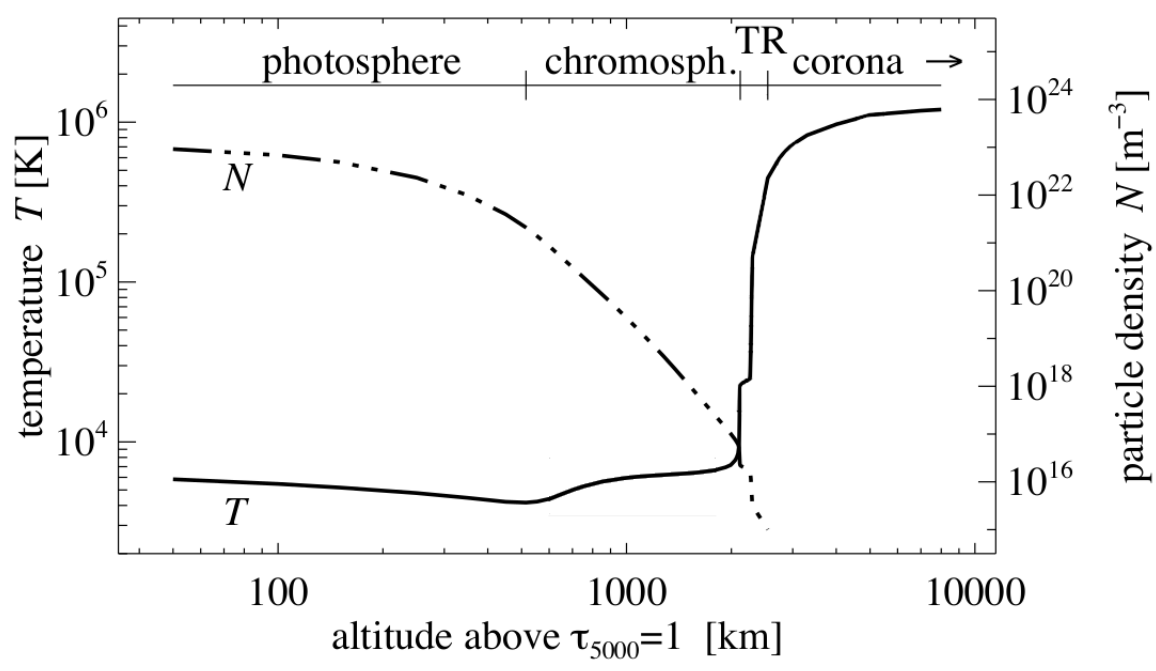

Figure 1.2: Solar atmosphere stratification as a function of height with the photosphere located at the altitude where the optical depth is unity (i.e. $\tau_{5000}=1$ ). Black solid line shows the temperature profile from the photosphere up to the corona. Dotted dashed line show the density profile in the solar atmosphere. The plot is motivated by the VAL (Vernazza, Avrett, Loeser) model (Vernazza et al. 1981). Image is courtesy of Hardi Peter.

moving away from a heat source, temperature should decrease monotonically. However, the solar corona is more than 100 times hotter than the photosphere. That is a direct violation of thermodynamics unless some other heating mechanism is operating.

To effectively heat the corona, a source of energy is required, that would balance the radiative losses and heat conduction. Many models have been proposed over the years. The purely hydrodynamic models were disproved since hydrodynamic waves and shocks cannot penetrate the dense layers of the chromosphere and transition region. Therefore, it is impossible to heat the corona. The alternative idea is based on magnetohydrodynamic (MHD) heating models. These models are divided into two categories. There are the alternating currents heating models (AC) and the direct current heating models (DC). Their difference lies in the timescales of the photospheric driver.

\subsubsection{AC heating}

It is known that waves carry energy and momentum in space and time. As mentioned before, hydrodynamic waves cannot reach and heat the corona. However, magnetohydrodynamic waves can reach the corona, and in principle, can dissipate some fraction of their energy and increase the coronal temperature. These MHD waves are excited from the interaction of the granular motion at the solar surface with the surface magnetic field. There are three different types of MHD waves, the Alfvén waves which propagate along the magnetic fieldlines, and the fast and slow magneto-acoustic waves that propagate perpendicular to the magnetic fieldlines. The magneto-acoustic waves are generally reflected or absorbed in the transition region, hence they cannot significantly affect the corona. On the other hand, Alfvén waves can penetrate the corona, thus they can deposit their energy to heat it and increase its temperature. Ohmic dissipation of the currents induced by these 

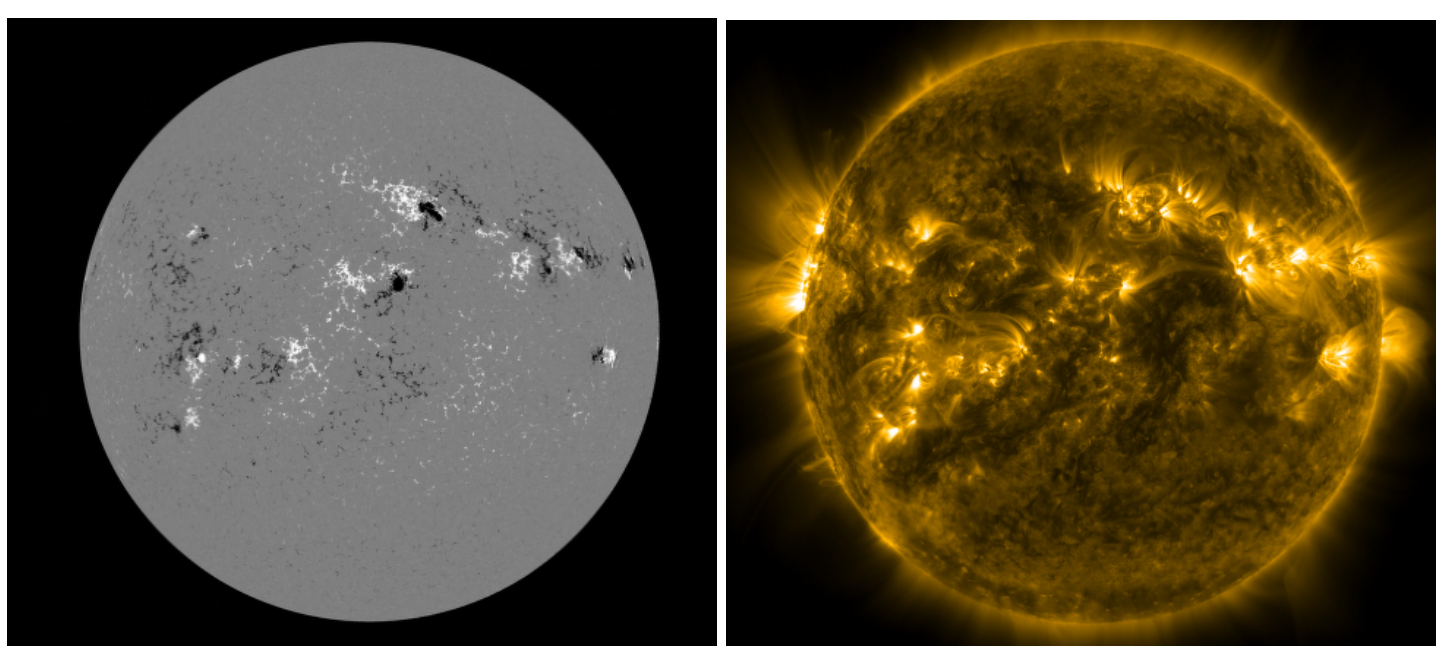

Figure 1.3: Full disk magnetogram and EUV emission observed from HMI and AIA/SDO on April 20 2015. The image is produced with the help of Helioviewer.org. Credits: SDO (NASA)

waves will convert the magnetic energy to thermal energy. Since the currents change direction on a faster time scale compared to the Alfvén time scale, this mechanism is called the alternating current mechanism

Direct observations of MHD waves in the corona were recently confirmed (Tomczyk et al. 2007). However, the work of Tomczyk et al. (2007) found that the observed energy flux of the Alfvén waves was not sufficient to explain the extremely high temperatures of the corona. On the other hand, numerical simulations of oscillating coronal loops which generate Alfvénic waves due to photospheric motions were reported to produce enough energy flux to heat the corona (van Ballegooijen et al. 2011).

\subsubsection{DC heating}

The other type of mechanism proposed for the coronal heating problem are the so called direct current (DC) models. The DC models can be distinguished into three main categories, namely the magnetic reconnection, stress-induced turbulence, and currents dissipation models. For the magnetic reconnection models, the magnetic fieldlines of coronal loops rooted in the photosphere are being twisted and braided by the random photospheric motions. This process is called fieldline braiding model, and it was proposed by Parker (1983). The twisted fieldlines (see Fig. 1 from Parker 1983) will build up enough nonpotential energy, which will be released by a storm of random reconnection events known as nanoflares (Parker 1988). The individual nanoflares will not have sufficient energy, but all the events along the loop combined should provide enough thermal energy to heat the corona. The twisting process of the fieldlines has a typical time scale which is much longer than the Alfvén crossing time along the loop. The second mechanism suggests that the random photospheric motions will lead a potential magnetic field to a non-potential. Thus currents will be induced and dissipated in the corona resulting in the overall heating. The dissipation process is known as the Ohmic dissipation of the induced currents. The last mechanism of stress-induced turbulence is based on a similar process as the dissipa- 
tion of currents. In this particular model, the energy cascades from large length scale to small length scales where most of the energy is dissipated. Finally, similar ideas such as the flux tube tectonics proposed by Priest et al. (2002) are being considered nowadays as well.

Due to the limitation of our current instruments, there is no confirmation of the existence of the nanoflares yet. This problem is expected to be resolved with the new space missions of the Solar Orbiter and the Parker Solar Probe that will observe the Sun at a close distance. On the other hand, 3D numerical simulations of large-scale models of active regions have already shown that the energy provided by the fieldline braiding model is sufficient to sustain a hot corona (Gudiksen and Nordlund 2005a; Bingert and Peter 2011; Warnecke and Peter 2019a). The DC mechanisms are considered by many researchers to be the most likely process responsible for the coronal heating (see the review from Klimchuk 2015, for more details).

\subsection{Solar-stellar connection}

The solar corona has been extensively studied for almost a century mainly by the space missions of Skylab, Yohokh, SOHO, and others. On the other hand, coronal observations of other stars are limited to a few number of stars. The solar and stellar coronal EUV and X-ray emission are a manifestation of the interaction between the surface magnetic field and the coronal plasma. The surface magnetic field plays a fundamental role in the evolution and dynamics of the solar and stellar coronae. However, an accurate estimation of the strength and the distribution of the surface magnetic field is only possible for our closest star, the Sun. Unfortunately, the spatial resolution of the current space instruments is limited to provide a more accurate measurement of the surface magnetic field for most stars. For a limited number of stars at close proximity to Earth, Zeemann Doppler Imaging (ZDI) and Zeemann broadening (ZB) techniques have been used to determine the magnetic field. For most stars, the surface magnetic field is impossible to estimate. For that reason, we have to rely on the indirect estimation of the magnetic activity of other stars by measuring, for example, their coronal X-ray emission. That will provide an insight into understanding the stellar coronae.

Disk integrated observations of other stars have been available thanks to the space mission of Chandra (Weisskopf et al. 2000), XMM-Newton (Jansen et al. 2001), and Einstein observatory (Giacconi et al. 1979). As in the solar case, the most prominent wavelength range suitable to study the corona are the EUV and X-ray regime. However, most of the EUV emission will be absorbed from the interstellar dust, making the EUV corona invisible from Earth. For that reason, the X-ray regime is chosen for the stellar coronal observations and diagnostics.

The first observations of other stars revealed coronal temperatures on the order of 20 MK or even 100 MK for some very active stars (Güdel 2004). These kinds of temperatures are 20 times higher than the ones observed in the solar corona. We assume that coronal heating in other stars will be primarily due to coronal loops rooted in active regions similar to the Sun. The length of these loops can vary for different stars depending on the stellar rotation rate or gravity acceleration. Using the Sun as a proxy might provide an understanding of the correlation between surface magnetism and coronal emission. 


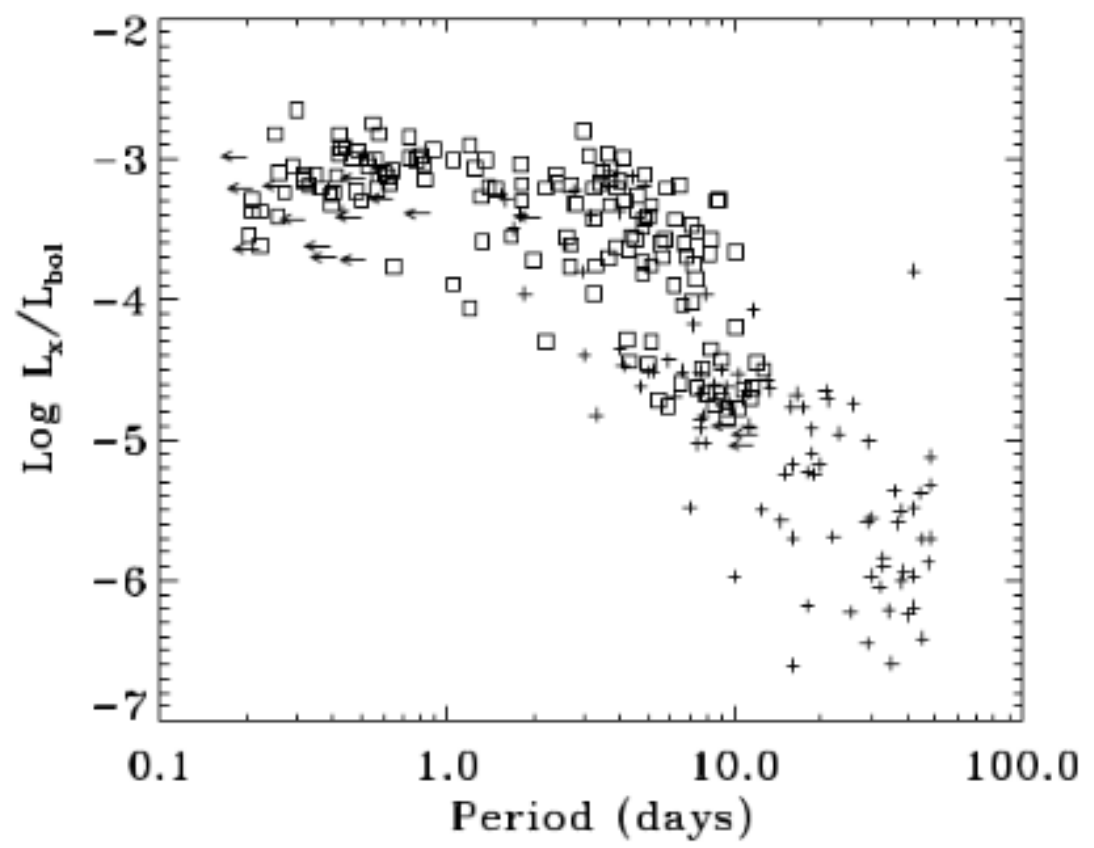

Figure 1.4: Normalised X-ray activity-rotation relation for a large sample of stars. Rotation increases going from right to left. X-ray emission increases going from bottom to top. The figure is taken from Pizzolato, N., Maggio, A., Micela, G., Sciortino, S., and Ventura, P., A\&A, 397, 147, 2003, reproduced with permission (C) ESO.

Correlations between observable quantities like X-ray, rotation rate, age, or even magnetic flux have been established mainly through observations of large samples of stars. These correlations could put constraints on our current coronal heating theories. That will prove to be a valuable tool for developing analytical models to describe the stellar coronal emission.

\subsubsection{Rotation-activity relation}

The solar and stellar magnetic field is generated by the plasma in the convection zone. The mechanism responsible for generating the surface magnetic field observed on the Sun can be partially explained by a dynamo theory. Even though the knowledge of the solar properties provides evidence to understand how this process operates on the Sun, this single G-type star cannot help us understand how the dynamo mechanism operates on others stars. For that, we need to investigate the behavior of different observable quantities from a large sample of stars that can be related to the underlying dynamo action.

Since the 70s, observations of other stars showed that the X-ray emission and stellar rotation rate are related. Main sequence stars, as they age, they lose angular momentum due to their stellar wind, and as a result, they tend to rotate slower. That is known as magnetic braking. The decrease of stellar rotation weakens the efficiency of the internal dynamo mechanism resulting in weaker coronal emission (Skumanich 1972). That indicates that age and thus stellar rotation determines the coronal activity of a star.

Using a large sample of stellar X-ray activity observations Pizzolato et al. (2003) 
found a rotation-activity relation connecting the normalised X-ray luminosity and the stellar rotation period as it is illustrated in Fig. 1.4. Two populations of stars can be distinguished (see Fig. 1.4). Stars with slow and intermediate rotation rate exhibit a power-law dependence of the X-ray emission to the rotation rate $L_{\mathrm{X}} / L_{\mathrm{bol}} \propto P^{-2}$. Here $L_{\mathrm{X}}$ is the coronal X-ray luminosity, $L_{\mathrm{bol}}$ is the bolometric luminosity and $P$ is the rotation rate of a star in days. For this specific regime, an increase in rotation results in a stronger dynamo action operating in the interior of a star. As a result, a stronger magnetic field is generated at the bottom of the convection zone, which then emerges because of buoyancy up to the photosphere. That leads to a larger number of active regions populating the photosphere, providing an excessive amount of heat in the corona. Finally, the increased heating leads to a substantial increase in the overall coronal X-ray emission. An interesting feature appears for rapid rotators at a rotation rate of $P \simeq 1$ days. There we observe the second population of stars, called the saturation regime. In this regime, the $\mathrm{X}$-ray emission is independent of the rotation period. The reasons for this saturation are not yet clear, and it is believed that saturation in the dynamo efficiency occurs at $P \simeq 1$ days (Reiners et al. 2014).

In this project, we are going to focus only on the non-saturated regime. The X-ray emission of stellar coronae is closely related to the surface magnetic field. We want to investigate how the magnetic field will affect the structure and dynamics of the X-ray corona. The underlying dynamo mechanism generating this surface magnetic field is outside the purpose of this project, and it will not be addressed here.

\subsubsection{Observed $\mathrm{X}$-ray activity to surface magnetic flux relation}

Observations of a large sample of stars revealed a correlation of the coronal X-ray emission not only with the stellar rotation rate but also with the surface magnetic flux (Vidotto et al. 2014). This correlation was already found in solar studies (see e.g. Fisher et al. 1998). They found, the coronal X-ray emission $L_{X}$ to follow a power-law relation as a function of the surface magnetic flux $\Phi$ (i.e. $L_{X} \propto \Phi^{m}$ ). The study was conducted by measuring the surface unsigned magnetic flux of a large number of solar active regions and it was related to the observed coronal X-ray emission. The power-law index was found to be slightly stronger than linear $(m=1.18)$.

Extending this to account also for other stars, Pevtsov et al. (2003) combined a large sample of observations from quiet Sun regions to other types of stars and T Tauri stars. They found that the observed coronal X-ray emission has a roughly linear dependence on the surface magnetic flux (see Fig. 1.5). More recent studies, on the other hand, suggest a much steeper power-law index $m$. Vidotto et al. (2014) from their large sample of stars with a different spectral type (F, G, K, M) place this index at $m=1.8$, while Kochukhov et al. (2020) focusing on a small sample of mainly $G$ type stars place this index at a larger value $m=2.7$. In general, the power-law index $m$ will be in the range of one to three. The large scatter of the index $m$ indicates a poor understanding of how the coronal X-ray emission is related to the surface magnetic flux for other stars. 


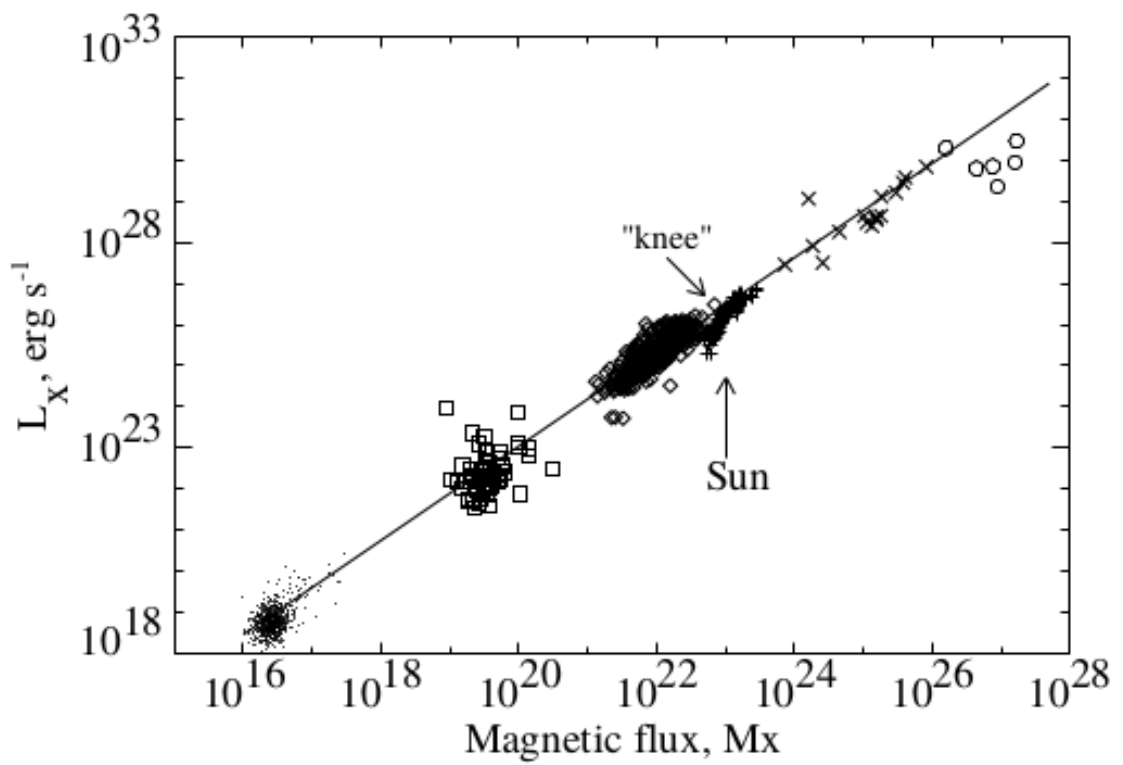

Figure 1.5: X-ray emission as a function of the total unsigned surface magnetic flux for solar and stellar observations. The dots represent quiet sun observations, squares are solar X-ray bright points, Diamonds are solar active regions, pluses are whole solar disk averages, crosses are other stars and circles are $\mathrm{T}$ Tauri stars. The solid line shows a power-law fit to the data with a power-law index 1.15. The figure is taken from Pevtsov, A.A, Fisher, G.H, Acton, L. W., Longcope, D. W., Johns-Krull, C. M., Kankelborg, C. C., and Metcalf, T. R.; The Relationship Between X-Ray Radiance and Magnetic Flux, The Astrophysical Journal, Volume 598, Issue 2, pp. 1387-1391, 2003, (C) AAS. Reproduced with permission .

\subsection{Motivation and aim of the present work}

The physical mechanism responsible for transporting the energy from the photosphere to the corona is still under debate. Until now, there is no clear evidence of how the corona is heated. The surface magnetic field, as it is discussed, proves to be an important factor in coronal heating and the general EUV and X-ray appearance of solar and stellar coronae.

Earlier observational studies have already established a connection between the coronal X-ray emission and the surface magnetic flux. This relationship was found to be a power-law, $L_{\mathrm{X}} \propto \Phi^{m}$. The power-law index $m$ has been estimated by numerous studies of the Sun and the stars and it was found to be in the range from one to almost three (see e.g. Fisher et al. 1998; Pevtsov et al. 2003; Vidotto et al. 2014; Kochukhov et al. 2020). Consequently, understanding the reasons of why this relationship between $L_{X}$ and $\Phi$ is a power-law and why there is a large range of $m$ found in observations, will also provide new insight into the stellar X-ray activity. Furthermore, a parameter study with different magnetic energy input, such as what is performed in this work, can put constraints on the probable coronal heating mechanism operating in the Sun and the stars. Finally, our work could potentially also provide an indirect way of estimating the surface magnetic flux of other stars which for most stars is not possible to observe.

Numerical models of the corona have been already applied in the solar case with great 
success, however, the stellar case is still an open field for exploration. The simulations presented in this work are based on the solar coronal model by Bingert (2009). This model is already well established and has been successful in reproducing some of the characteristics of solar coronal loops (Bingert and Peter 2011; Warnecke and Peter 2019a). As a result, it is a well-suited model to use for a parameter study by changing the amount of surface magnetic flux at the bottom boundary of the computational domain. With this, we can represent stars more active than the Sun and investigate the effect on the coronal $\mathrm{X}$-ray emission. However, a parameter study, such as the one performed in this work, was not possible in the past. The large computational time required was a limiting factor to perform a large number of simulations (see Chap. 4). After the recent implementations of a non-Fourier heat flux scheme and the Boris correction (see Warnecke and Bingert 2020), a parameter study is now possible. We investigate the $L_{X} \propto \Phi^{m}$ through a simple analytical model and a series of 3D MHD numerical experiments.

The layout of the thesis is as follows. In Chap. 2 we give a brief introduction of the magnetohydrodynamic (MHD) equations. We also derive the RTV scaling laws. We finish by describing the advantages of large-scale 3D numerical simulations of coronal loops. In Chap. 3 we describe the analytical model we developed to explain the $L_{\mathrm{X}} \propto \Phi^{m}$ based on the RTV scaling laws. In Chap. 4 we give a brief description of the solar coronal model we use for our numerical simulations. In Chap. 5 and Chap. 6 we show the results from the simulations where the surface magnetic flux is changed to represent stars more active than the Sun. In Chap. 7 we summarise our results and discuss their implication to stellar coronal activity. 



\section{Theoretical framework}

The Sun, the stars, and most of the astrophysical objects are subject to the motion and dynamics of plasma. In the single-fluid picture, plasma can be described by a set of partial differential equations known as the magnetohydrodynamics (MHD) equations. These equations can be found in many textbooks regarding plasma physics (see e.g. Priest 2014). In this chapter, we summarise the basic equations and their implication.

\subsection{Electromagnetic equations}

\subsubsection{Maxwell's equations}

Motions in the plasma are strongly affected by the interaction with the electric and magnetic field $(\boldsymbol{E}, \boldsymbol{B})$. The fields $\boldsymbol{E}$ and $\boldsymbol{B}$ are described by Maxwell's equations,

$$
\begin{aligned}
\boldsymbol{\nabla} \cdot \boldsymbol{E} & =\frac{\rho_{e}}{\epsilon_{0}} \\
\boldsymbol{\nabla} \cdot \boldsymbol{B} & =0 \\
\boldsymbol{\nabla} \times \boldsymbol{E} & =-\frac{\partial \boldsymbol{B}}{\partial t} \\
\boldsymbol{\nabla} \times \boldsymbol{B} & =\mu_{0} \boldsymbol{j}+\epsilon_{0} \mu_{0} \frac{\partial \boldsymbol{E}}{\partial t}
\end{aligned}
$$

where $\rho_{e}$ is the charge density, $\epsilon_{0}$ is the vacuum's permittivity, and $\mu_{0}$ is the vacuum's magnetic permeability. The parameters $\epsilon_{0}$ and $\mu_{0}$ are related to the speed of light as $c=\frac{1}{\sqrt{\epsilon_{0} \mu_{0}}}$.

For the majority of plasma phenomena observed in astrophysics, plasma motion can be considered as the motion of a fluid. The simplest approach is the single-fluid approximation, where we can make a few assumptions. (I) It is assumed that the plasma is macroscopically quasi-neutral with an equal number of negative $n_{e}$ and positive ions $n_{i}$. Thus, $\rho_{e}=n_{e} e-n_{i} e \simeq 0$. (II) In general, the motion of the plasma can be considered to have a single macroscopic velocity $u$, density $\rho$, and pressure $p$. (III) Furthermore, for most of the phenomena related to solar physics, the velocities can be considered non-relativistic, $u<<c$.

In the simplest form of MHD, it is assumed that the plasma is a perfect conductor with zero resistivity. This is known as ideal MHD, and the magnetic field is "frozen" in the plasma. The ideal MHD is applicable only when, (I) the plasma is collisional and the particles follow a Maxwellian distribution, (II) the resistivity is zero, thus there is no diffusion, and (III) typical length scales in the plasma are larger than the Debey length and 
Larmor radius. However, in some astrophysical objects such as the Sun, resistivity is not necessarily zero thus there is a deviation from ideal MHD. In this case, the plasma is also subject to diffusion. Even if the plasma is not a perfect conductor, but the other conditions are still valid, we can use, what is called, a resistive MHD. The finite resistivity can create current sheets that describe phenomena such as magnetic reconnection, turbulence, and others that cannot be explained with ideal MHD. Finally, if the plasma is no longer collisional and the particle distribution is not a Maxwellian, then the MHD breaks down, and there is a need to employ kinetic theory.

In the framework of ideal MHD, the displacement current $\frac{\partial \boldsymbol{E}}{\partial t}$ of Eq. (2.4) is negligible. We define the typical plasma length scale $l$ and a time scale $\tau$ with $\tau \simeq l / u$. The left hand side of Eq. (2.4) can be estimated,

$$
|\boldsymbol{\nabla} \times \boldsymbol{B}| \simeq \frac{B}{l}
$$

The electric field $\boldsymbol{E}$ of the right hand side of Eq. (2.4) can be expressed by using Ohm' law

$$
\boldsymbol{j}=\sigma(\boldsymbol{E}+\boldsymbol{u} \times \boldsymbol{B})
$$

For an ideal MHD, the electric conductivity $\sigma \rightarrow \infty$ therefore,

$$
\boldsymbol{E}=-\boldsymbol{u} \times \boldsymbol{B} .
$$

Using now the characteristic length and time scales we get,

$$
E \simeq u B \rightarrow \frac{\partial E}{\partial t} \simeq \frac{u B}{\tau}=\left(\frac{B}{l}\right) u^{2}<<c^{2} .
$$

Since the displacement current scales quadratically with velocity, then for non-relativistic phenomena this term can be ignored. For a quasi-neutral plasma (i.e. $\rho_{e} \simeq 0$ ) the Maxwell's equation reduce to,

$$
\begin{aligned}
\boldsymbol{\nabla} \cdot \boldsymbol{E} & =0 \\
\boldsymbol{\nabla} \cdot \boldsymbol{B} & =0 \\
\boldsymbol{\nabla} \times \boldsymbol{E} & =-\frac{\partial \boldsymbol{B}}{\partial t} \\
\boldsymbol{\nabla} \times \boldsymbol{B} & =-\mu_{0} \boldsymbol{j} .
\end{aligned}
$$

\subsubsection{Induction equation}

It is widely common to substitute the electric field $\boldsymbol{E}$ by using Ohm's law and combining it with the Maxwell's equations (see Eq. (2.10)-Eq. (2.12)) to derive the induction equation,

$$
\frac{\partial \boldsymbol{B}}{\partial t}=\boldsymbol{\nabla} \times(\boldsymbol{u} \times \boldsymbol{B})-\boldsymbol{\nabla} \times(\eta \boldsymbol{\nabla} \times \boldsymbol{B}),
$$

where $\eta=1 /\left(\mu_{0} \sigma\right)$ is the magnetic resistivity. The resistivity $\eta$ generally depends on temperature. The induction equation Eq. (2.13) shows that the temporal evolution of the magnetic field $\boldsymbol{B}$ is due to the induction of the magnetic field because of the plasma motion 
(i.e. $\boldsymbol{\nabla} \times(\boldsymbol{u} \times \boldsymbol{B}))$ and the diffusion of the magnetic field because of the resistivity (i.e. $\boldsymbol{\nabla} \times(\eta \nabla \times \boldsymbol{B}))$.

In numerical simulations it is more convenient to solve for the vector potential $\boldsymbol{A}$ instead of the magnetic field $\boldsymbol{B}$. The reason is that the vector potential automatically satisfies Maxwell's second law (see Eq. (2.11)),

$$
\boldsymbol{\nabla} \cdot \boldsymbol{B}=0 \rightarrow \boldsymbol{B}=\boldsymbol{\nabla} \times \boldsymbol{A} .
$$

Assuming now a constant resistivity $\eta$, the induction equation (i.e. Eq. (2.13)) can be expressed as,

$$
\frac{\partial \boldsymbol{A}}{\partial t}=\boldsymbol{u} \times(\boldsymbol{\nabla} \times \boldsymbol{A})+\eta \boldsymbol{\nabla}^{2} \boldsymbol{A} .
$$

Here we use the resistive gauge $\phi=\eta \boldsymbol{\nabla} \cdot \boldsymbol{A}$. We distinguish two extreme cases. In the first case, $\eta=0$ (i.e. ideal MHD). For this case, we consider the plasma to be a perfect conductor. This means that there is no diffusion of the magnetic field. The magnetic fieldlines are frozen in the plasma, and they are dragged around due to the plasma motion. In the alternative case, $\boldsymbol{u}=0$. For this case, the plasma is not moving, and the magnetic field is only subject to diffusion and eventually will decay.

\subsubsection{Poynting theorem}

The Poynting flux describes the flux of electromagnetic energy through a unit area per unit time. It is defined as,

$$
\boldsymbol{S}=\frac{1}{\mu_{0}} \boldsymbol{E} \times \boldsymbol{B} \quad\left[\frac{W}{m^{2}}\right] .
$$

Substituting the electric field $\boldsymbol{E}$ through the Ohm's law (see Eq. (2.6)) we rewrite the previous equation as,

$$
\boldsymbol{S}=\eta(\boldsymbol{j} \times \boldsymbol{B})-\frac{1}{\mu_{0}}(\boldsymbol{u} \times \boldsymbol{B}) \times \boldsymbol{B} .
$$

where $\eta=1 / \mu_{0} \sigma$. The above equation describes the Poynting flux as the interaction of the currents with the magnetic field (i.e. $\boldsymbol{j} \times \boldsymbol{B}$ ) and the interaction of the plasma flows with the magnetic field (i.e. $\boldsymbol{u} \times \boldsymbol{B} \times \boldsymbol{B}$ ).

Both terms of Eq. (2.17) contribute in the change of the magnetic energy to other forms of energy. The magnetic energy density is defined as,

$$
e_{m}=\frac{\boldsymbol{B}^{2}}{2 \mu_{0}} \quad\left[\frac{J}{m^{3}}\right]
$$

If we now take the time derivative we get,

$$
\frac{\partial e_{m}}{\partial t}=\frac{1}{\mu_{0}} \boldsymbol{B} \cdot \frac{\partial \boldsymbol{B}}{\partial t}
$$

We can substitute the time derivative of the magnetic field by using the induction Eq. (2.13),

$$
\frac{\partial e_{m}}{\partial t}=\frac{1}{\mu_{0}} \boldsymbol{B}[\boldsymbol{\nabla} \times(\boldsymbol{u} \times \boldsymbol{B})-\boldsymbol{\nabla} \times \eta(\boldsymbol{\nabla} \times \boldsymbol{B})] .
$$


Using now the definition of the Poynting flux Eq. (2.17) and Ampere's law we get our final result,

$$
\frac{\partial e_{m}}{\partial t}+\boldsymbol{\nabla} \cdot \boldsymbol{S}=-\eta \mu_{0} \boldsymbol{j}^{2}-\boldsymbol{u} \cdot(\boldsymbol{j} \times \boldsymbol{B}) .
$$

If the right hand side of Eq. (2.21) was zero then the magnetic energy would be a conserved quantity. In that case the magnetic energy would flow in space but since there are no sinks and sources there will be no loss of energy.

In the case where we have sinks and sources then the right hand side of Eq. (2.21) describes the conversion of magnetic energy into other form of energies. The first term indicates the loss of magnetic energy by Ohmic dissipation which converts into thermal energy. The second term, indicates the conversion of the magnetic energy into kinetic energy through the work done by the Lorentz force.

\subsection{Fluid equations}

\subsubsection{Continuity equation}

In the limit of MHD, plasma can be described as the motion of a quasi-neutral fluid. The plasma mass density is a conserved quantity, and it flows in space in a continuous way (thus the name continuity equation). Mathematically the mass conservation is described by the continuity equation,

$$
\frac{\partial \rho}{\partial t}+\nabla \cdot(\rho \boldsymbol{u})=0
$$

where $\rho$ is the mass density and $\boldsymbol{u}$ is the plasma velocity. Eq. (2.22) states that an increase of density at some point requires a mass flow into the neighboring region and a density decrease requires a mass flow out of the surrounding region. Alternatively, Eq. (2.22) can be expressed using the Langragian derivative $\frac{D}{D t}=\frac{\partial}{\partial t}+\boldsymbol{u} \cdot \boldsymbol{\nabla}$ as,

$$
\frac{D \rho}{D t}+\rho \boldsymbol{\nabla} \cdot \boldsymbol{u}=0
$$

For an incompressible flow, $\boldsymbol{\nabla} \cdot \boldsymbol{u}=0$ and,

$$
\frac{D \rho}{D t}=0
$$

\subsubsection{Momentum equation}

The fluid motion is subject to forces acting on it. Hence, Newton's second law describes the motion of the fluid. The equation of motion can be expressed with the momentum equation,

$$
\rho \frac{D u}{D t}=\sum_{i} f_{i}
$$

where $D / D t$ is the Lagrangian derivative and $f_{i}$ are the forces per unit volume. More precisely, this forces are, 
- the pressure gradient $-\nabla p$

- the Lorentz force $\boldsymbol{j} \times \boldsymbol{B}$

- the viscous force $2 v \boldsymbol{\nabla} \circ(\rho \underline{S})$

- gravitational force $-\rho \nabla \Phi$,

where $v$ is the viscosity and $\underline{S}$ is the strain tensor. Forces that arise from rotation, such as Coriolis and centrifugal force, are generally not taken into account in the coronal study, and thus they are ignored.

\subsubsection{Equation of state}

To fully describe a fluid it is required to establish an equation of state. For most astrophysical objects that consist of plasma at low pressure, the equation of state of an ideal gas,

$$
p=\frac{k_{B}}{\mu m_{p}} \rho T
$$

can be considered as a good approximation. Here $\mu$ is the atomic weight, $k_{B}$ is the Boltzmann constant and $m_{p}$ is the proton's mass. For a fully ionised hydrogen plasma, like the one we find in the solar corona, we have the same amount of protons and electrons, therefore $\mu=0.5$ and for the densities,

$$
n=n_{e}+n_{p}=2 n_{e}, \quad \rho=n_{p} m_{p}+n_{e} m_{e} \simeq n_{e} m_{p} .
$$

\subsubsection{Energy equation}

The last equation necessary to fully describe the plasma evolution is the energy equation,

$$
\rho T \frac{D s}{D t}=-\mathcal{L}
$$

where $s$ is the entropy per unit mass and $\mathcal{L}$ are all the sources and sinks of energy. The previous equation shows that an increase of heat per unit volume as it flows in space is only due to the total effect of all the sinks and sources. In the absence of sinks and sources, entropy is a conserved quantity.

A more convenient way to express Eq. (2.28) is by using the rate of temperature change $T$ instead of entropy $s$. To do that, we have to introduce the change of the internal energy $d e$,

$$
d e=T d s-\frac{p}{\rho^{2}} d \rho .
$$

Now Eq. (2.28) can be expressed as a function of the internal energy,

$$
\rho \frac{D e}{D t}-\frac{p}{\rho} \frac{D \rho}{D t}=-\mathcal{L}
$$

For an ideal gas the internal energy is given by $e=c_{v} T$, with $c_{v}$ being the specific heat for a constant volume. Using now the continuity Eq. (2.23) we derive the final expression, 


$$
c_{v} \rho \frac{D T}{D t}+p \boldsymbol{\nabla} \cdot \boldsymbol{u}=-\mathcal{L}
$$

The sources and sinks are described in the following section.

\subsubsection{Ohmic dissipation}

The term that describes the conversion of magnetic energy to thermal energy is called Ohmic or Joule dissipation. The expression is given by,

$$
\mathcal{L}_{H}=-\eta \mu_{0} \dot{j}^{2}
$$

where $\eta$ is the magnetic resistivity and for the solar corona it is on the order of $1 \mathrm{~m}^{2} / \mathrm{s}$. However, following the work of Bingert and Peter (2011) we have, for numerical reasons, to set a much larger value of $\eta$ in the numerical simulations (see Sect.4.2.1). The Ohmic heating term has a significant contribution in the heating of the coronal plasma in our numerical models.

\subsubsection{Viscous heating}

The viscous force can be considered to act analogously as friction does in our everyday life, converting the kinetic energy of a body into heat. Similarly, for fluids, the conversion of kinetic energy into heat is given by the viscous heating term,

$$
\mathcal{L}_{v}=-2 \rho v \underline{S}^{2},
$$

where $v$ is the kinematic viscosity and typical values in the solar corona, from transport theory, are on the order of $10^{10} \mathrm{~m}^{2} / \mathrm{s}$. The $\underline{S}$ is the rate of strain tensor,

$$
\underline{S}=\frac{1}{2}\left(\frac{\partial u_{i}}{\partial x_{j}}+\frac{\partial u_{j}}{\partial x_{i}}-\frac{2}{3} \delta_{i j} \boldsymbol{\nabla} \cdot \boldsymbol{u}\right)
$$

\subsubsection{Spitzer heat conduction}

The energy transport in the solar corona is described by the heat conduction. The heat flux vector along the magnetic fieldlines reads,

$$
\boldsymbol{q}=-K \nabla T
$$

where $K$ is the thermal conduction tensor. The minus sign indicates that the heat flux points downwards to parts with lower temperatures and is proportional to the temperature gradient. The steeper the gradients, the more efficient it is. The heat is transported by the electrons, and for this term to hold, the electrons mean free path should be much smaller than the temperature scale height, which is true for the corona.

For the fully ionised plasma of the solar corona, the heat conduction tensor $K$ has the general form,

$$
K=K_{\perp} \delta_{i j}+\left(K_{\|}-K_{\perp}\right) \hat{b}_{i} \hat{b}_{j}
$$

where $K_{\perp}, K_{\|}$are the perpendicular and parallel coefficients and $\hat{b}_{i}, \hat{b}_{j}$ are the unit vectors along the magnetic fieldlines. For the components $i$ and $j$, the perpendicular component 
of the heat conduction $K_{\perp}$ in the solar corona is by many orders of magnitude weaker compared to the parallel component $K_{\|}$. Thus, we set $K_{\perp}=0$ and the heat flux reads,

$$
\boldsymbol{q}=-K_{\|} \hat{\boldsymbol{b}}(\hat{\boldsymbol{b}} \cdot \nabla T)
$$

For fully ionised gas Spitzer (1962) gives,

$$
K_{\|}=K_{0} T^{5 / 2} \quad\left[\frac{W}{m K}\right]
$$

where typical coronal values yield $K_{0}=1.8 \times 10^{-10} \mathrm{~W} \mathrm{~m}^{-1} \mathrm{~K}^{-7 / 2}$. To conclude, the Spitzer heat conduction term in the energy equation is the divergence of Eq. (2.37), thus,

$$
\mathcal{L}_{s}=\boldsymbol{\nabla} \cdot \boldsymbol{q}=-K_{0} \boldsymbol{\nabla} \cdot\left(T^{5 / 2} \hat{\boldsymbol{b}}(\hat{\boldsymbol{b}} \cdot \boldsymbol{\nabla} T)\right)
$$

The efficiency of the Spitzer heat conduction poses a serious numerical challenge mainly because it can increase the computational time tremendously. In Chap. 4 we are going to describe a numerical method in order to overcome this problem.

\subsubsection{Radiative cooling}

An equally important process that takes place also in the solar corona is radiative cooling. The corona is considered to be optically thin. The intensity $I(\lambda)$ of an optically thin spectra line produced by photons at a specific wavelength $\lambda$ via spontaneous emission is expressed as,

$$
I(\lambda)=\int A_{X} G\left(T, \lambda, n_{e}\right) n_{e} n_{H} d z
$$

where, $A_{X}$ is the elemental abundance in the corona, $G\left(T, \lambda, n_{e}\right)$ is the contribution function which can be calculated through the CHIANTI (Dere et al. 1997) atomic database and $n_{e}, n_{H}$ are the number densities of electron and hydrogen respectively. To further continue the analysis of Eq. (2.40) we need the density profile along the line of sight, which gives rise to differential emission measure. The coronal abundances are calculated based on the work from Meyer (1985), Murphy (1985), Cook et al. (1989).

For the optically thin corona, the radiative losses are not coupled to the radiation field, and hence it can be derived by integrating Eq. (2.40) in a specific temperature range. The expression is given by,

$$
\mathcal{L}_{R}=n_{e} n_{H} Q(T)
$$

The function $\mathrm{Q}(\mathrm{T})$ is called the radiative loss function with units of $\mathrm{Wm}^{3}$. For the implementation in the code see Bingert (2009).

To conclude, radiative cooling is most efficient for lower temperatures and higher density, thus it is significant in the lower, more dense parts of the corona and transition region. 


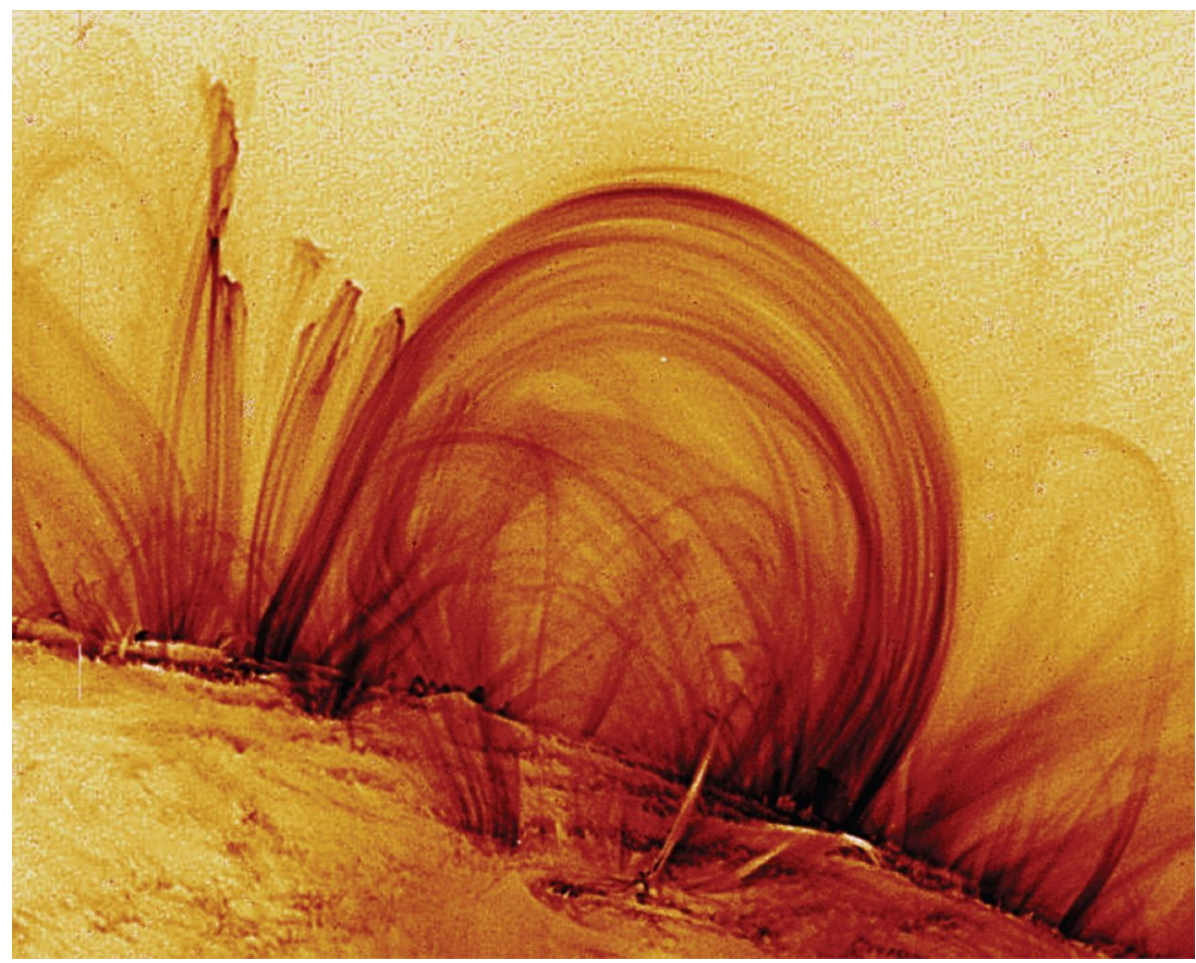

Figure 2.1: Coronal loops in the solar atmosphere as observed by the Transition Region and Coronal Explorer (TRACE) instrument on November 6th 1999. The image shows the EUV emission of the hot coronal plasma $(T \simeq 1 \mathrm{MK})$ confined inside the loop. The colors in this picture are inverted. Credit: NASA/LMSAL.

\subsection{Basic characteristics of coronal loops}

The low optical emission of the solar corona compared to the photosphere makes it difficult to study it in the optical wavelength range. However, because of the high temperatures in the corona, the most prominent wavelength range used as a coronal diagnostic is in the EUV and X-ray regime. The first EUV and X-ray observations revealed a solar corona consisting of a fully ionised gas, which is confined along the magnetic fieldlines, that form what is known as coronal loops. These coronal loops have a semicircular shape with the two sides anchored in the photosphere and consist of numerous magnetic fieldlines. In Fig. 2.1 it is shown the structure of hot coronal loops in the solar atmosphere as it was observed by Transition Region and Coronal Explorer (TRACE). It is clear that when the plasma confined inside the coronal loops is heated to temperatures beyond 1 MK, it becomes much brighter than the surrounding ambient plasma revealing this semicircular structure. The coronal loops are considered the main building blocks of the solar atmosphere, and they play an important role in the coronal heating problem.

Coronal loops observed in the Sun might vary in size, temperature, and density. The length scales vary from $1 \mathrm{Mm}$ for X-ray bright points, and it can reach up to $100 \mathrm{Mm}$ for typical active regions. Their temperature varies from a few $10^{5} \mathrm{~K}$ for cool loops, and it can increase to a few MK for active regions loops. Finally, the number density is in the range from $10^{14}$ to $10^{16} \mathrm{~m}^{-3}$.

The coronal loops act as a bridge for the magnetic energy to propagate from the pho- 
tosphere up to the corona, which will convert into heat. Analytical models of coronal loops have been developed based on a few physical assumptions. (I) It is assumed that the solar corona has a high conductivity. In this case, the coronal plasma can be considered to be frozen in the magnetic field. The plasma cannot move from one fieldline to another, but it is confined to a movement only along the fieldline. (II) The plasma $\beta$ is assumed to be much smaller than unity in the solar corona. The plasma $\beta$ is defined as $\beta=p / p_{m}$, where $p$ is the gas pressure and $p_{m}$ is the magnetic pressure. For $\beta>1$, the gas pressure dominates. As a result, the magnetic fieldlines will be dragged around by the plasma flows. For the solar corona with $\beta<1$, the magnetic pressure dominates, and coronal loops behave like rigid bodies. The plasma flow will only be following the shape of the loop. (III) The heat conduction, as discussed in Sect.2.2.4.3, is only parallel to the magnetic fieldlines. The heat conduction perpendicular to the fieldlines is negligible, and therefore the magnetic fieldlines inside the coronal loops can be considered thermally isolated by the neighboring magnetic fieldlines (see e.g. Priest 2014; Reale 2014).

\subsubsection{One dimensional coronal loop models}

Solar coronal loops consist of numerous thin strands of magnetic fieldlines. Each one of them can evolve independently. The motion of the plasma under typical coronal conditions is confined along the magnetic fieldlines. In this framework, it is common to assume a constant loop cross-section and neglect the effect of curvature, non-uniform loop geometry, or waves. Consequently, a simple approach is to consider the coronal loops as one-dimensional (1D) structures. Its dynamics and evolution can be described by solving the 1D time-dependent hydrodynamic equations numerically (see e.g. Hansteen 1993; Antiochos et al. 1999; Bradshaw and Cargill 2006). Namely, the continuity, momentum, and energy equation. In this configuration, the magnetic field does not explicitly affect the plasma flows but only acts as a pathway to the coronal plasma motion. This consideration makes the study of the coronal loops much simpler.

As any other computational method of describing a physical system, there are advantages and disadvantages. One important advantage of 1D models is the relatively high spatial resolution that can be achieved. This allows for capturing some aspects of these 1D coronal loops with much greater detail. For example, the steep temperature gradients in the transition region can be resolved well in 1D models (Serio et al. 1981). Furthermore, the time-dependent solutions of the hydrodynamic questions provide an insight into the evolution of plasma properties such as temperature or velocity along the loop. Knowing the evolution of loop properties can be used for a direct comparison with actual solar coronal observations. On the other hand, one important limitation of the 1D models is the ad hoc prescription of a heating function. This cannot be treated self-consistently in the environment of a 1D setup, but a more realistic three-dimensional (3D) approach is required. However, the 1D models can be directly compared with solar observations and provide quantitative information on the different heat input functions prescribed (see e.g. Reale et al. 2000). More detailed information on 1D coronal loop models can be found in the review paper of Reale (2014). In the next section, we will to describe a specific case of 1D models for static coronal loops. This will give rise to the well-known RTV scaling laws (Rosner et al. 1978) which play a significant role throughout this thesis. 


\subsubsection{Energy balance}

The first 1D coronal loop models developed examine static coronal loops in hydrostatic equilibrium. To fully describe the evolution and dynamics of coronal plasma, the MHD equations need to be solved. However, for static coronal loops, the plasma moves as a steady-state incompressible flow along the magnetic fieldlines. In this case, $\boldsymbol{\nabla} \cdot \boldsymbol{u}=0$ and the time derivatives vanish. The energy equation (see Eq. (2.31)) is governed only by the balance among heat conduction, heat input and radiative cooling,

$$
H-L_{\mathrm{rad}}-\boldsymbol{\nabla} \cdot \boldsymbol{q}=0 .
$$

Integrating Eq. (2.42) in the whole coronal volume and assuming a vanishing heat flux at the boundaries, the total heat input is balanced by the energy loss through the radiative cooling term. The heat conduction term redistributes the energy from the hot parts of the corona to the cooler ones. The modeled 1D corona loops can sustain a million Kelvin degree plasma for long time scales, consistent with the behavior of actual solar coronal loops.

\subsubsection{RTV scaling laws}

The relation between temperature, density, and pressure of coronal loops has been the main focus of many 1D loop models. The early study of Rosner, Tucker \& Vaiana (Rosner et al. 1978, ; RTV) assumed 1D coronal loops in hydrostatic equilibrium. Their model considered coronal loops with a constant heating rate and a constant cross-section. Using these simple physical assumptions and the energy balance equation, they derived the famous RTV scaling laws that relate the coronal temperature and pressure with the heating rate and the coronal loop length. Alternatively, these scaling laws can be expressed to relate thermodynamic quantities, such as temperature and density, with the loop characteristics such as the heating rate and length.

The first scaling law discussed relates the temperature at the loop apex with the heating rate $H$ and the loop length $L$. The coronal loop is considered to have approximately a semi-circular shape, and we only account for derivatives along the loop. The energy balance for a semi-circular loop is,

$$
-\frac{1}{A} \frac{d}{d s}(A q)-L_{\mathrm{rad}}+H=0
$$

where $q$ is the heat flux, and the loop coordinate $s$ goes from 0 to the apex at $L$ for a symmetric loop. The cross-section of the loop $A$ is considered to be independent of the coordinates in this framework. Finally, the heating rate $H$ is also considered to be constant. Most of the energy is radiated at the transition region, therefore above the footpoints of the coronal loop, the radiative losses can be neglected. The heating rate $H$ is only balanced by the heat conduction,

$$
H=\frac{d q}{d s} .
$$

The heat flux is expressed with the formula (see also Eq. (2.39)),

$$
q=-K_{0} T^{5 / 2} \frac{d T}{d s} .
$$


The Eq. (2.44) can be integrated from a position $s$ to the apex of the loop $L$,

$$
\int_{s}^{L} H d s^{\prime}=-\int_{s}^{L} d\left(K_{0} T^{5 / 2}\left(s^{\prime}\right) \frac{d T\left(s^{\prime}\right)}{d s^{\prime}}\right)
$$

The solution of the integral yields,

$$
H(L-s)=-\left.\left[K_{0} T^{5 / 2}\left(s^{\prime}\right) \frac{d T\left(s^{\prime}\right)}{d s^{\prime}}\right]\right|_{s^{\prime}=s} ^{s^{\prime}=L} .
$$

Since the loop is considered to be thermally isolated, the heat flux should vanish at the apex of the loop. Thus,

$$
\left.\frac{d T}{d s}\right|_{s=L}=0
$$

By using the above boundary condition we obtain,

$$
H(L-s)=K_{0} T^{5 / 2} \frac{d T}{d s}
$$

Solving Eq. (2.49) from 0 to $L$ we obtain the final result,

$$
H \frac{L^{2}}{2}=\frac{2 K_{0}}{7} T_{\max }^{7 / 2}
$$

Here we ignored the temperature at the footpoints since it is negligible compared to the apex temperature. Our final result will be,

$$
T_{\max } \propto H^{2 / 7} L^{4 / 7} \quad \text { 1st RTV scaling law }
$$

The second RTV scaling law relates the number density $n_{e}$ with the heating rate $H$ and loop length $L$ and can be derived similarly to temperature $T$. At the loop footpoints, all the incoming heat flux $q$ is radiated away by the radiative cooling term $L_{\text {rad }}$ while the local heating rate $H$ does not contribute to the energy balance equation and it can be neglected. Hence,

$$
L_{\mathrm{rad}}=-\frac{d q}{d s}
$$

The optically thin radiative losses can be parameterized through the radiative loss function $Q(T)$

$$
L_{\mathrm{rad}}=n_{e}^{2} Q(T),
$$

where $n_{e}$ is the electron's number density. For the lower corona and transition region, the radiative loss function can be approximated as a power-law (see e.g. Rosner et al. 1978; Priest 1982),

$$
Q(T)=\chi T^{-1 / 2} \quad \text { with } \quad \chi=10^{-32} \mathrm{Wm}^{3} \mathrm{~K}^{1 / 2} .
$$

Later studies (Cook et al. 1989) provide a steeper dependence by including the contribution of $\mathrm{Fe}$ as $Q(T) \propto T^{-2 / 7}$. However, the difference is not significant, and we can therefore consider Eq. (2.54) to be a good approximation of the radiative losses. The energy balance reads, 


$$
n_{e}^{2} \chi T^{-1 / 2}=-\frac{d q}{d s}
$$

We multiply the above equation with $K_{0} T^{5 / 2}$ and using the chain rule,

$$
K_{0} \chi n_{e}^{2} T^{2}=\underbrace{-K_{0} T^{5 / 2} \frac{d T}{d s}}_{\mathrm{q}} \frac{d q}{d T}=\frac{d}{d T}\left(\frac{q^{2}}{2}\right)
$$

The Eq. (2.56) can be integrated along the loop and we obtain,

$$
K_{0} \chi n_{e}^{2} \int_{0}^{L} T^{2} d T=\int_{0}^{L} d\left(\frac{q^{2}}{2}\right) \rightarrow \frac{2 K_{0} \chi n_{e}^{2}}{3}\left[T^{3}(L)-T^{3}(0)\right]=q^{2}(L)-q^{2}(0)
$$

Using again the boundary condition for the heat flux $(q(L)=0)$ and that $T(L)>>T(0)$ we get our final result,

$$
q(0)=-\sqrt{\frac{2 K_{0} \chi n_{e}^{2}}{3}} T_{\max }^{3 / 2},
$$

which yields the relation between the heat flux at the coronal base with the temperature at the apex of the loop. The heat conduction will transfer all the energy from the apex into the base of the loop. Thus,

$$
q(0)=-H L \text {. }
$$

substituting Eq. (2.58) and the first RTV scaling law Eq. (2.51), yields the final result,

$$
n_{e} \propto H^{4 / 7} L^{1 / 7}, \quad \text { 2nd RTV scaling law }
$$

Both scaling laws, Eq. (2.51) and Eq. (2.60) show a dependence of the coronal temperature and density on the incoming heating rate $H$, and as a first case, we consider a loop with a fixed-length $L$. If the incoming heating rate at the base of the coronal loop is increased, then the heat conduction is expected to increase as well. The increased heat conduction will redistribute the extra energy. This will lead to an increase in the peak temperature at the apex of the loop. At the same time, radiation has to increase to balance the enhanced heat input, leading to an increase in the coronal density at the loop base. For the alternative case we consider coronal loops with a fixed heat input $H$, but the length can vary. In this case, a change in temperature and density is only regulated by a variation in the loop length $L$. Longer loops will experience lower peak temperature and density since they have to redistribute the energy in a larger volume for a given fixed heating rate.

We conclude that a proper study of coronal loops requires not to achieve the correct high peak temperature but to get the proper coronal density. This will produce the correct coronal emission in comparison to actual observations. The RTV scaling laws provide an essential tool in the study of solar coronal loops. In addition, they are also widely considered in stellar studies (see e.g. Güdel 2004, section 11). 


\subsubsection{Three dimensional numerical models of coronal loops above ac- tive regions}

EUV and X-ray observations of the solar corona revealed a coronal plasma with a temperature of $1 \mathrm{MK}$ or even more. The mechanism which heats the corona from a few thousand Kelvin of the photosphere to one million Kelvin of the corona is still not well understood. 1D models provided some insight into the problem but, to properly capture the complex nature of coronal loops, 3D simulations are necessary.

One of the models proposed to explain the coronal heating problem is the fieldline braiding model (Parker 1972, 1983). In this model, the braiding of the magnetic fieldines because of the photospheric velocities will release magnetic energy in the corona leading to high temperatures. One of the first attempts to realistically model the solar corona based on the fieldline braiding mechanism was the 3D model of Gudiksen and Nordlund (2002) based on the STAGGER code (Galsgaard and Nordlund 1996). The model simulates a part of the corona above an active region, including a photospheric driver and a Spitzer heat conduction along the magnetic fieldlines. They found that even though the coronal magnetic field in the corona is close to potential, the photospheric motions can provide enough energy to heat coronal loops to high temperatures. Synthetic EUV emission from their model shows a good comparison with actual coronal loops as observed with AIA/SDO or TRACE (see e.g. Peter et al. 2004).

An improved version of the STAGGER code accounting also for the convection zone is the widely used BIFROST code (Gudiksen et al. 2011). The BIFROST code simulates the solar corona but also includes the convection zone leading to a realistic self-consistent photospheric velocity distribution. Furthermore, the code solves for the full radiative transfer equations, and, also a hydrogen ionization module accounts for the non-local thermodynamic equilibrium of the solar chromosphere.

An equally important code used is the MuRAM code (Vögler et al. 2005). Initially, the MuRAM code was developed to simulate the magneto-convective motions in the solar photosphere. Recently Rempel (2017) extended the ability of the code to study the solar corona as well. Similar to BIFrost code, the MuRAM code also includes the convection zone creating the photospheric granular motion self-consistently. However, the chromosphere is considered to be in local thermodynamic equilibrium for MuRAM code.

A limited number of other codes can be found in the literature, such as the RADMHD (Abbett 2007) where they prescribe the heating term ad hoc based on observations. There is also the work of Mok et al. (2005), Mok et al. (2008) that study the formation of coronal loops by thermal instabilities. Finally, van der Holst et al. (2014) developed the AWSoM code that studies the ability of Alfvén waves to heat the corona.

In this work we use the solar coronal model developed by Bingert (2009) using the PENCIL CODE (Brandenburg et al. 2020). This model is based on the early work of Gudiksen and Nordlund (2002). It has been proved successful in the description of the solar corona by either including an observed magnetogram of an active region together with a velocity driver to mimic the granular motion (Bingert and Peter 2011; Bourdin et al. 2013) or in flux emergence experiments (Feng 2015). One of the key results of this model is the synthetic emission in the EUV that was found to match quite well the solar coronal loops observed with the AIA/SDO at the $171 \AA$ channel (Peter and Bingert 2012; Bourdin et al. 2016; Warnecke and Peter 2019a) (see Fig. 2.2b,c). Furthermore, 

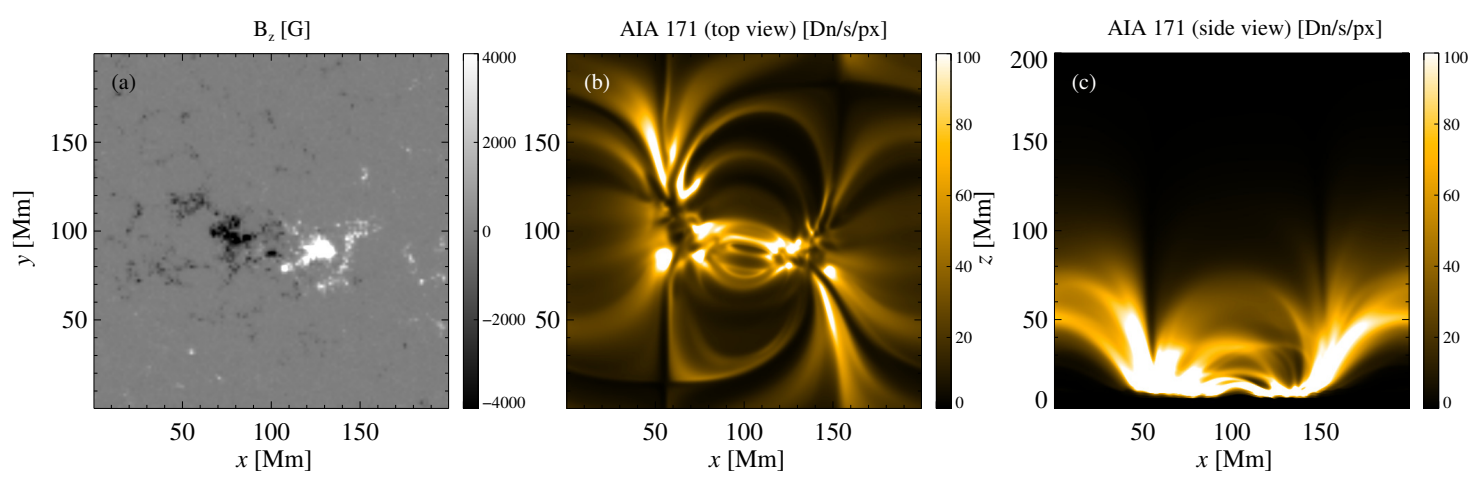

Figure 2.2: Active region magnetogram from the numerical models used in this thesis and synthetic emission of coronal loops. Panel a): $B_{z}$ magnetic field at the bottom boundary of the simulation box. Panel b): Top view of the synthetic EUV emission at the $171 \AA$ channel. Panel c): Side view of the $171 \AA$ A synthetic emission. The units of the EUV emission are in DN/s/px.

3D models allow for a more detailed study of loop properties such as temperature and density distribution and how they compare with theoretically expected values (such as the RTV scaling laws derived in Sect. 2.3.1.2) that were found in ideal descriptions of coronal loops. Studying these scaling laws from the observational point of view is challenging, and computational power has been a limiting factor until recently. One of the first studies testing the RTV scaling laws and their limitations was by Bourdin et al. (2016). Their model included a small solar active region as an input to the bottom boundary. They showed that the temperature and density for a collection of magnetic fieldlines agree well with the predicted values from the RTV scaling laws. Finally, Warnecke and Peter (2019b) based on this model studied the influence of the magnetic helicity on the coronal X-ray emission of active stars. They found that the coronal X-ray emission increases as a powerlaw with the magnetic helicity without changing the strength of the photospheric magnetic field.

To conclude, 3D models of coronal loops provide the best realistic approach to study the solar corona. These models self-consistently treat the heat input in the corona, and they are the best tool at our disposal to study the complex structure and dynamic behavior of coronal loops. In this work, based on a 3D coronal model, we employ a parameter study to explain the stellar coronal activity. We present a series of simulations and investigate how the change of the magnetic energy input injected directly from the photosphere affects the coronal X-ray emission.

\subsubsection{Stellar coronal loops}

Non-solar X-ray instruments have shown that most other stars emit strongly in X-rays, which is a clear sign of an active corona. The stellar corona is often much stronger than the solar one, and the reasons for that are still an open question. Studies have shown that age is an essential factor for creating an active corona. Younger stars that tend to rotate much faster than the Sun produce a much stronger X-ray corona (see e.g. Telleschi et al. 2005). Due to the large distance between the stars in our galaxy, it is impossible 
to spatially resolve their corona. Thus, we cannot obtain information on the size or the appearance of stellar coronal loops. For that reason, we rely only on indirect evidence.

One way of studying stellar coronal loops is by examining explosive events like stellar flares. The study of stellar X-ray flares involves plasma confined in loop-like structures, which after eruption enhance the X-ray emission signature significantly (Reale et al. 2002). This allows putting constraints on various characteristics, such as their size and temperature. It was found that typical stellar coronal loops vary in length from similar to the solar ones (Reale et al. 1988), and they can reach up to the order of the solar radius (Getman et al. 2008).

A slightly different approach is to study the X-ray emission from the whole stellar disk. It is shown that various structures on the solar surface, such as quiet sun regions, active regions, flares, and others, affect the corona differently. This method can then be used on other stars and explain the stellar activity by applying weight on the different components and obtain information regarding the coronal heating problem (Peres et al. $2001,2004)$. More information can be found in the reviews of Reale (2014) and Güdel (2004).

Observations can also be used to find correlations between coronal X-ray emission and stellar parameters such as the surface magnetic field. Unfortunately, these relations are only obtained through observations. In this work, we fill in this gap by building an analytical model and explain these observed correlations. 



\section{Stellar coronal $\mathrm{X}$-ray emission and surface magnetic flux}

* Chap. 3 reproduces the article Stellar coronal X-ray emission and surface magnetic flux by J. Zhuleku, J. Warnecke, and H. Peter published in Astronomy \& Astrophysics, 640, A119 (2020), reproduced with permission (c) ESO, DOI: 10.1051/0004-6361/202038022. The abstract and appendix are not included. I wrote the first draft of the paper and developed the initial stages of the model.

\subsection{Introduction}

The Sun, other solar-like stars, and, in particular, other more active stars are sources of $\mathrm{X}$-ray emission. These X-rays are mostly of a thermal nature and originate from stellar coronae due to the high temperatures, well above $1 \mathrm{MK}$, in their outer atmospheres. Observational studies show a clear dependence of the X-ray emission on the surface magnetic field for individual structures on the Sun as well as for stars as a whole. Combining measurements from the Sun and other stars, Pevtsov et al. (2003) found this dependence to be slightly steeper than linear following roughly a power law, $L_{X} \propto \Phi^{1.15}$. Here $L_{X}$ and $\Phi$ are the X-ray luminosity and the unsigned surface magnetic flux. Different studies found different power-law relations, depending on the structures and stars that were investigated. For example, studying the X-ray emission and the surface magnetic field of solar-like stars, Kochukhov et al. (2020) found a relation of $L_{X} \propto \Phi^{2.68}$. Observations of different

Table 3.1: Observed relations of X-ray luminosity and X-ray flux to surface magnetic flux and magnetic field.

\begin{tabular}{lccl}
\hline \hline & $\begin{array}{c}\text { index } m \text { in } \\
L_{\mathrm{x}} \sim \Phi^{m}\end{array}$ & $\begin{array}{c}\text { index } p \text { in } \\
F_{\mathrm{x}} \sim B^{p}\end{array}$ & Reference \\
\hline Objects & & & \\
Solar active regions & 1.19 & & $\begin{array}{l}\text { Fisher et al. (1998) } \\
\text { Solar X-ray bright points }\end{array}$ \\
Solar microflares & 0.89 & & $\begin{array}{l}\text { Longcope et al. (2001) } \\
\text { Solar disk averages }\end{array}$ \\
Solar disk averages & & 1.48 & Kirichenko and Bogachev (2017) \\
Solar-like stars (mostly G type) & 2.68 & $1.5-2.2$ & Wolfson et al. (2000) \\
Low mass stars (F, G, K, M) & 1.80 & & Konevolenskaya (2007) \\
Sun and large sample of stars & 1.15 & & Vidotto et al. (2014) \\
\hline & & & Pevtsov et al. (2003) \\
\hline
\end{tabular}


solar magnetic structures, such as active regions, bright points, or microflares, and of stars with various levels of activity, reveal power-law relations between X-ray emission and the magnetic field. Mostly the power-law indices range from about one to two (see Table 3.1 for a non-complete list). There is quite a large scatter in the X-ray observations of other stars, in part because the data usually used for a statistical analysis of the X-ray emission might capture different phases of stellar activity (e.g., Vidotto et al. 2014).

On the Sun, most of the total X-ray emission originates from coronal loop systems, and it is widely assumed to be also true for other (solar-like and more) stars (e.g., Güdel 2004). The general properties of these loops can be described using the Rosner, Tucker \& Vaiana (RTV) scaling laws, named after the authors of the original study (Rosner et al. 1978). These scaling relations connect the temperature and pressure of a loop to the (volumetric) heating rate and the length of the loop through power laws and will be described in more detail in Sect. 3.4. To derive the scaling laws, one usually assumes a onedimensional coronal loop in hydrostatic equilibrium with a constant volumetric heating rate where the loop length is smaller than the pressure scale height. An analytical analysis of the balance between energy input, heat conduction, and radiative cooling then yields the scaling laws (e.g., Sect. 6.5.1A of Priest 1982). Even though they were developed for simple static coronal loops, the RTV scaling laws still capture the average properties of quite complex situations as found in three-dimensional coronal models (Bourdin et al. 2016). The RTV scaling laws have also been used extensively in stellar coronal studies (e.g., Güdel 2004) and can thus be considered as a basis for our understanding of stellar coronae.

The RTV scaling relations require some information on the heating rate (and the loop length) to determine the temperature and pressure (and thus the density) of a loop. The exact form of the mechanism to heat a stellar corona to temperatures in excess of $1 \mathrm{MK}$ is still open to debate. In our study, we employ two widely used proposals, mainly for illustrative purposes, namely the Alfvén wave model (e.g., van Ballegooijen et al. 2011) and the nanoflare or field-line braiding model (Parker 1972, 1983). For both scenarios, the upward-directed Poynting flux, and by this, the heating rate can be scaled as a function of the surface magnetic field (see Sect. 3.3). With that scaling of the energy input with the surface magnetic field, we have the critical input to derive the temperature and density from the RTV scaling relations.

Based on the temperature and density of a loop one can estimate the X-ray emission to be expected from the structure. Under coronal equilibrium conditions, essentially, the optically thin emission is proportional to the density squared, and is a function of temperature, often called the temperature response function or contribution function (e.g., Del Zanna and Mason 2018). Using the appropriate atomic data, one can then calculate the X-ray emission over a given wavelength region, for the continuum emission alone (Culhane 1969) and also including emission lines (Landini and Monsignori Fossi 1970). For different wavelength regions the temperature response functions will be different, with emission from shorter wavelength intervals having the tendency to originate from hotter plasma (e.g., Mewe and Gronenschild 1981, their Fig. 3; or Mewe et al. 1985, their Fig. 1). In order to evaluate the temperature response for a given instrument one should use a modern atomic data base tool (e.g., Chianti; Dere et al. 1997) and the wavelength dependence of the effective area of the instrument. We employ both in our considerations in Sect. 3.2. 
In this study, we use the temperature response function of various X-ray detectors (Sect. 3.2) and two of the main coronal heating mechanisms (Sect. 3.3) together with the RTV scaling laws (Sect. 3.4) to derive an analytical model describing how the X-ray emission depends on the unsigned surface magnetic flux. Finally, in Sect. 3.5 we compare our model with stellar observations and discuss the consequences for stellar surface magnetic fields as well as for stellar coronal heating mechanisms.

\subsection{Temperature dependence of $\mathrm{X}$-ray radiation}

Optically thin X-ray radiation is a combination of emission lines and continua that both change with the temperature of the source region. In general, both line and continuum emission are also proportional to the (electron) density squared, so that

$$
F_{\mathrm{X}}=n^{2} R(T),
$$

where $F_{\mathrm{X}}$ is the loss of energy (per volume and time) through optically thin X-ray radiation, and $n$ is the number density. The function $R(T)$ characterizes the temperature dependence. When considering only one single emission line, this would be the contribution function, typically including collisional excitation rates, ionization fraction, and other factors. When considering the total emission from a number of lines (plus the continuum), $R(T)$ would essentially be the sum of all contribution functions involved. Then, one has to consider that these lines are spread over a wavelength region and hence one has to account for the efficiency of the instrument as a function of wavelength. In those cases, $R(T)$ is usually called temperature response, and we will use this term in the remainder of this paper.

To calculate the temperature response for a number of X-ray instruments we use the Chianti atomic data package (v9.0.1; Dere et al. 1997, 2019). We first calculate the radiances of the emission lines in a range of wavelengths $\lambda$ from $0.1 \AA$ to $250 \AA$ for an isothermal plasma at temperature $T$. For this we employ the CHIANTI routine ch_synthetic.pro. In the second step we use make_chianti_spec.pro to calculate the resulting spectrum $I_{T}(\lambda)$ in this same wavelength range, which also includes the calculation of the continua. We do this for a number of temperatures $T$ in the range from $\log _{10} T[\mathrm{~K}]=5.5$ to 8.0. For the calculation of the spectra, we use the standard CHIANTI ionization equilibrium and photospheric abundances. In the final step we multiply the spectrum at each temperature by the effective area $A_{\text {eff }}(\lambda)$ of a number of instrumentfilter-detector combinations (see Table 3.2). Here we use the values as stored in Chianti ${ }^{1}$. The response at temperature $T$ is then simply given by the integral of intensity and effective area over wavelength,

$$
R(T)=\int I_{T}(\lambda) A_{\text {eff }}(\lambda) \mathrm{d} \lambda
$$

Typically, the response of an X-ray instrument peaks at temperatures around (or slightly below) $10 \mathrm{MK}$. For lower temperatures the response drops quickly (see Fig. 3.1). This is

\footnotetext{
${ }^{1}$ In the Chianti database: dbase/ancillary_data/instrument_responses/. For Hinode/XRT, ChIANTI does not list effective areas so we use values supplied in the XRT branch of SolARSofT (www. Imsal.com/ solarsoft).
} 


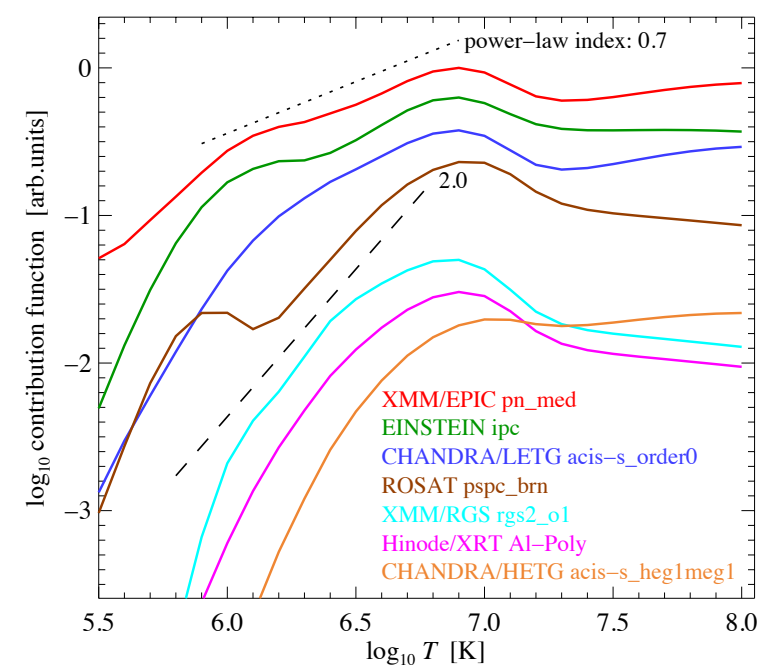

Figure 3.1: Temperature response $R(T)$ for several X-ray instruments. The naming convention for the detector-filter combinations corresponds to the CHIANTI database (also see Table 3.2). Each curve is multiplied by a constant to get the curves nicely into the same plot. For comparison, we plot two power laws with power-law indices of 0.7 (dotted) and 2.0 (dashed). See Sect. 3.2.

the case for a wide range of X-ray instruments, including major instruments for stellar observations, such as XMM-Newton (Jansen et al. 2001), Chandra X-ray observatory (Weisskopf et al. 2000), Röntgensatellit (ROSAT; Pfeffermann et al. 1986), and Einstein observatory (Giacconi et al. 1979). The current main instrument for solar studies behaves in a similar way (Hinode/XRT; Golub et al. 2007a).

Coronae of the Sun and other stars harbor mostly plasma in the range from about $1 \mathrm{MK}$ to $10 \mathrm{MK}$. To implement the temperature response into a power-law estimate (in Sect. 3.4) we consider a simplified variant. The change of the temperature response below $10 \mathrm{MK}$ is reasonably well characterized by a power-law fit,

$$
R(T) \propto T^{\alpha},
$$

with a power-law index $\alpha$. We apply a power-law fit to each of the instruments in a temperature range from $\log _{10} T[\mathrm{~K}]=5.9$ to 6.9 and list the resulting power-law indices in Table 3.2. Only for the ROSAT case, the lower limit (in $\log _{10} T$ ) is 6.2 to avoid the bump at low temperatures. In general, the power-law indices $\alpha$ range from 0.7 to about 2 (see sample power laws in Fig. 3.1), with few exceptions giving also indices $\alpha$ of 3 or more. When considering the (often many) different filters of one single instrument, power-law indices $\alpha$ are found in the same range. As an example, we show XMM filters in appendix.

Based on the above discussion for a wide range of instrument-filter-camera combinations we can conclude that in general a power law as in Eq. (3.3) is a reasonable fit to the temperature response functions. In general, the power-law indices range from $\alpha=0.7$ to 2 . 
Table 3.2: Overview of selected X-ray instruments and the resulting power-law indices.

\begin{tabular}{|c|c|c|c|c|c|}
\hline \multirow[b]{2}{*}{ Instrument } & \multirow[b]{2}{*}{ detector/filter } & \multirow[b]{2}{*}{$\begin{array}{c}\text { energy range } \\
{[\mathrm{keV}]}\end{array}$} & \multirow{2}{*}{$\begin{array}{l}\text { power-law } \\
\text { index } \alpha \text { for: } \\
\quad R \propto T^{\alpha}\end{array}$} & \multicolumn{2}{|c|}{$m$ for: $L_{\mathrm{X}} \propto \Phi^{m}$} \\
\hline & & & & $\begin{array}{c}\text { nanoflares } \\
(\beta=2)\end{array}$ & $\begin{array}{c}\text { Alfvén waves } \\
\quad(\beta=1)\end{array}$ \\
\hline XMM/EPIC & pn_med & $0.05-12$ & $0.7 \pm 0.03$ & $1.0 \pm 0.3$ & $0.8 \pm 0.2$ \\
\hline EINSTEIN & ipc & $0.1-5$ & $0.7 \pm 0.04$ & $1.0 \pm 0.3$ & $0.8 \pm 0.2$ \\
\hline CHANDRA/LETG & acis-s_order0 & $0.07-10$ & $1.2 \pm 0.1$ & $1.1 \pm 0.3$ & $0.9 \pm 0.2$ \\
\hline ROSAT & pspc_brn & $0.1-2.3$ & $1.6 \pm 0.1$ & $1.2 \pm 0.3$ & $0.9 \pm 0.2$ \\
\hline XMM/RGS & rgs2_o1 & $0.3-2.5$ & $1.8 \pm 0.2$ & $1.3 \pm 0.3$ & $1.0 \pm 0.3$ \\
\hline Hinode/XRT & Al-poly & $0.2-3$ & $2.1 \pm 0.2$ & $1.3 \pm 0.4$ & $1.0 \pm 0.3$ \\
\hline CHANDRA/HETG & acis-s_heg1meg 1 & $0.4-10$ & $3.1 \pm 0.3$ & $1.6 \pm 0.4$ & $1.2 \pm 0.3$ \\
\hline
\end{tabular}

\subsection{Magnetic field and heating of coronal plasma}

The plasma in the corona of the Sun and other stars is heated to temperatures of well above $1 \mathrm{MK}$. In view of the scaling laws to be discussed in Sect. 3.4, we first consider how to relate the heat input into the corona to the magnetic field on the surface of the Sun or a star. For this we consider two of the main heating mechanisms, namely Alfvén wave heating (van Ballegooijen et al. 2011) and field-line braiding (or nanoflare heating Parker 1972, 1983). In order to get a scaling of the energy flux into the upper atmosphere, the Poynting flux, we follow the discussion in Fisher et al. (1998). In general, one can relate the Poynting flux in the vertical direction, $S_{z}$, to the vertical surface unsigned magnetic field $B$ by

$$
S_{z} \propto B^{\beta} \quad \text { with } \begin{cases}\beta=2: & \text { braiding / nanoflares, } \\ \beta=1: & \text { Alfvén waves. }\end{cases}
$$

In the case of braiding, the magnetic field $\boldsymbol{B}$ at the surface is driven by convective flows with a velocity $\boldsymbol{v}$. Neglecting resistivity, the Poynting flux $\boldsymbol{S}=-(\boldsymbol{v} \times \boldsymbol{B}) \times \boldsymbol{B} / \mu_{0}$ in the vertical direction can be approximated by $S_{z} \propto v B^{2}$ (Fisher et al. 1998, Eq. 3), where $v$ is the horizontal photospheric velocity. Hence the exponent $\beta=2$ in Eq. (3.4) for field-line braiding (or nanoflares).

In the case of an Alfvén wave propagating into the corona, the wave energy flux is given by $\rho\left\langle v^{2}\right\rangle v_{\text {prop }}$, with density $\rho$, mean square velocity amplitude $\left\langle v^{2}\right\rangle$, and the propagation speed being the Alfvén speed, $v_{\text {prop }}=v_{\mathrm{A}}$. Because the Alfvén speed is proportional to the magnetic field $B$, so is the energy flux of the Alfvén wave (Fisher et al. 1998, Eq. 2). Hence the exponent $\beta=1$ in Eq. (3.4) for Alfvén waves.

In the remainder of this study, we will use the values of $\beta=1$ and 2 just to represent the possible ranges of what we might expect for different heating processes. Other possible parameterizations have been suggested and used, such as $\beta=1.75$ based on magnetohydrodynamics (MHD) turbulence models (Rappazzo et al. 2008; van Wettum et al. 2013), or $\beta=1$ derived from full-sun visualizations through 1D parameterized models (Schrijver et al. 2004).

For the scaling laws discussed in Sect. 3.4 the volumetric heating rate $H$ is required. If all the Poynting flux $S_{z}$ injected through the bottom boundary is dissipated in the corona, then the dissipated energy integrated in height should equal the Poynting flux at the bottom, that is, $S_{z}=\int H \mathrm{~d} z$. This has been shown to be the case in 3D MHD models (e.g., 
Bingert and Peter 2011). If the volumetric heating $H$ is constant, then $S_{z}=H L$, where $L$ is the length (or height) of the coronal structure. To be more general, we allow the influx of energy, the Poynting flux, to be related by a power law to the heating rate,

$$
H L \propto S_{z}^{\gamma} .
$$

In the remainder of this study we will assume $\gamma=1$, but will keep $\gamma$ in the equations.

Finally, we have to relate the surface unsigned magnetic field strength $B$ to the unsigned magnetic flux $\Phi$ in the region considered, for example, a coronal bright point, an active region, or a whole star. If we consider $B$ to be the average magnetic field strength, then the magnetic flux would be given by

$$
\Phi=B A,
$$

where $A$ is the (weighted) area of the respective region (using the same terminology as Fisher et al. 1998). The area of an active region can be related to the magnetic flux through a power law as

$$
A \propto \Phi^{\delta} \quad \text { with } \delta=0.819 .
$$

If $\delta$ were unity, this would imply that the (average) magnetic field strength in each active region is the same. In their analysis of observed solar magnetograms, Fisher et al. (1998) found a value of $\delta=0.819$ (their Sect. 4.1.3, following their Eq. 18). This value of $\delta<1$ implies that larger active regions have a magnetic flux that is large not only because of the greater area coverage, but also because the (peak or average) magnetic field strength is higher. We will discuss the special cases of $\delta=0$ and $\delta=1$ in Sect. 3.5.2

Interestingly, Eq. (3.7) is also roughly valid for other stars. In a study of solar-like stars, Kochukhov et al. (2020) found a similar power-law relation based on the filling factor $f$ and the averaged surface magnetic field $\langle B\rangle$ as $f \propto\langle B\rangle^{\delta}$ with $\delta=0.86$ (see their Fig. 8). This filling factor $f$ is defined as the ratio of the surface area covered by a magnetic structure (e.g., active region) $A$ to the total surface of a star $A_{\text {star }}$. Then with Eq. (3.6), this relation can be rearranged to $A \propto A_{\text {star }}^{(1-\delta)} \Phi^{\delta}$. Since $\delta \simeq 1$ we can ignore $A_{\text {star }}$ and retrieve the same equation as Eq. (3.7).

A similar conclusion can be drawn from a study of stars with different spectral types and activity levels (See et al. 2019). In that study, the estimated filling factor $f$ using the large-scale surface magnetic field and total surface magnetic flux follows a similar powerlaw relation as in the work of Kochukhov et al. (2020) but with $\delta=0.78$. In conclusion, these results of stellar observations provide further support for using Eq. (3.7) with $\delta=$ 0.819 in our model.

To estimate the length $L$ of the coronal structure, we assume that this is related to the square root of the area $A$, that is, to the linear scale of the region considered,

$$
L \propto A^{1 / 2} .
$$

Basically, this consideration assumes that the separation length of two (main) magnetic polarities of opposite sign in the active region is proportional to the linear extent of the active region. In the case of the Sun this can be confirmed through observations (see, e.g., Cameron et al. 2010, their Fig. 1). We will discuss this limitation in Sect. 3.5.3.

With the relations in Eqs. (3.4) to (3.8) we can find how the heating rate $H$ and length scale $L$ depend on the (average) magnetic field $B$ or the magnetic flux $\Phi$. This and the 
discussion in Sect. 3.2 will allow us in the following to derive a scaling between the X-ray emission and the surface magnetic flux.

\subsection{Scaling laws: coronal emission vs. magnetic flux}

The thermal properties of coronal loops, their temperature, density, and pressure structure were described in 1D models more than 40 years ago. An early key finding that still is a pillar of coronal physics are the so-called RTV scaling laws. These relate the length $L$ and (volumetric) heating rate $H$ of a loop to its temperature $T$ and pressure $p$ (Rosner et al. 1978). In this section, we will employ these scaling laws together with the discussions in Sects. 3.2 and 3.3 to derive a scaling between X-ray emission and surface magnetic flux.

The original scaling laws presented by Rosner et al. (1978) are $T \propto(p L)^{1 / 3}$ and $H \propto p^{7 / 6} L^{-5 / 6}$. They are commonly known as the RTV scaling laws named after the initials of the authors. Essentially, these can be derived by comparing energy input, energy redistribution through heat conduction, and radiative losses (see, e.g., Priest 1982, Sect. 6.5).

The RTV scaling relations can be rearranged to express temperature and density in terms of heating rate and loop length,

$$
\begin{aligned}
& T \propto H^{2 / 7} L^{4 / 7}, \\
& n \propto H^{4 / 7} L^{1 / 7} .
\end{aligned}
$$

Here we used the number density $n$ through the ideal gas law, $n \propto p / T$. While originally derived for static 1D loops, these scaling laws still give a good representation in more complex situations. For example, these RTV relations capture quite well the average properties of time-dependent 3D MHD models of an active region (Bourdin et al. 2016).

Observations show that the coronal density $n$ depends on the stellar rotation rate $\Omega$. While the RTV scaling laws do not explicitly take into account this dependency, they implicitly include it. The heating rate $H$ depends on the surface magnetic field $B$ (see Eqs. (3.4) and (3.5)), which itself depends on the stellar rotation rate $\Omega$. Hence, through Eq. (3.10) the coronal density depends implicitly on rotation and thus would change from star to star. We assume $B \propto \Omega^{1}$, which is representative of observations that give a range of power-law indices from 0.7 to 1.3 (Kochukhov et al. 2020; Vidotto et al. 2014). Together with Eqs. (3.4), (3.5), and (3.10) this yields $n \propto \Omega^{0.57}$ (for $\beta=\gamma=1$ and neglecting the dependence on the length $L)$. Thus, for Alfvén wave heating $(\beta=1)$ this model result is consistent with observations by Ivanova and Taam (2003) who found a power-law relation $n \propto \Omega^{0.6}$. Thus we conclude that our model properly treats the change in the coronal density due to the variation of stellar activity introduced by rotation, even though only implicitly.

We can now derive the relation between X-rays and (surface) magnetic field. In the first step we express the X-ray emission $F_{\mathrm{X}}$ as given in Eq. (3.1) through magnetic field $B$ and length of the loop structure $L$. For this we use Eq. (3.3) to replace the temperature response and substitute the temperature and density from Eqs. (3.9) and (3.10). Using then Eqs. (3.4) and (3.5) we can replace the (volumetric) heating rate by the magnetic 
field strength. This yields

$$
F_{\mathrm{X}} \propto B^{p} L^{q} \quad \text { with }\left\{\begin{aligned}
p & =\frac{\beta \gamma}{7}(2 \alpha+8), \\
q & =\frac{1}{7}(2 \alpha-6) .
\end{aligned}\right.
$$

With the values of $\alpha$ listed in Table 3.2, mostly $|q|$ is much smaller than 0.5. Consequently, the X-ray emission $F_{\mathrm{X}}$ is mainly dependent on the magnetic field $B$ but only weakly depends on the length $L$ of the coronal structure because $\beta, \gamma \geq 1$. This result for $F_{\mathrm{X}}$ essentially applies for a single structure, for example, one coronal loop.

In the second step, we express the total X-ray luminosity $L_{X}$ in terms of the surface magnetic flux $\Phi$. The total X-ray loss $L_{X}$ from a region on the Sun (the X-ray luminosity in the case of a whole star) is given by integrating the X-ray emission $F_{\mathrm{X}}$ over the respective area $A$ (or the whole star). Assuming that $F_{\mathrm{X}}$ is constant (or represents an average value), we simply have

$$
L_{\mathrm{X}}=F_{\mathrm{X}} A \text {. }
$$

If $A$ is considered to be the surface of a whole star, Eqs. (3.7) and (3.8) are not necessarily applicable. However, we expect the surface area of a star contributing to the X-ray luminosity to obey a similar relation as an active region (see Eq. (3.7)).

Substituting Eqs. (3.6), (3.7), and (3.8) into Eqs. (3.11) and (3.12) yields our final result,

$$
\begin{aligned}
& L_{\mathrm{X}} \propto \Phi^{m} \quad \text { with } \\
& m=\frac{\beta \gamma}{7}(2 \alpha+8)+\delta\left(\frac{4}{7}+\frac{1}{7} \alpha-\frac{8}{7} \beta \gamma-\frac{2}{7} \alpha \beta \gamma\right) .
\end{aligned}
$$

Technically, $L_{X}$ in Eq. (3.12) represents the X-ray luminosity per unit length and needs to be integrated along the line of sight to get the total X-ray luminosity. However, choosing an appropriate length scale to perform the line of sight integration is not trivial. There are at least two natural choices for the length scale. One way would be to use the coronal pressure scale height, which is proportional to the coronal temperature $T$. Multiplying Eq. (3.13) by the pressure scale height and replacing the temperature in a similar way as before will add two extra terms in each of the two brackets in Eq. (3.13). This will, however, change the power-law index $m$ by only roughly $5 \%$ for both heating models. Compared to the uncertainty range in $m$ (cf. Table 3.2) we consider this insignificant. Another possibility to account for the line of sight integration would be to multiply Eq. (3.13) with the coronal loop length $L=A^{1 / 2}$. This would add $0.5 \delta$ to $m$ in Eq. (3.13). In that case, the changes in $m$ are larger, around $30 \%$ to $40 \%$ higher for both heating models. Still this would be comparable to the uncertainty range of $m$. Overall, we conclude that the line of sight integration will not significantly alter the quantitative results for the power-law indices $m$. Hence, we can consider $L_{\mathrm{X}}$ roughly independent of the integration along the line of sight and Eq. (3.13) a valid expression for the total X-ray luminosity.

The power-law indices $m$ resulting from Eq. (3.13) are listed in Table 3.2 for different X-ray instruments, that is, their different temperature responses parameterized by $\alpha$ (Sect. 3.2), and for two different choices of the heating mechanism $(\beta=2$ for nanoflares and $\beta=1$ for Alfvén waves). In Table 3.2 we keep $\gamma=1$ (cf. Eq. 3.5) and use $\delta=0.819$ as found in observations of the Sun and solar-like stars (see Eq.3.7). 
The overall errors in the power-law index $m$ are on the order of $20 \%$ to $40 \%$ (see Table 3.2). We estimated these errors from the uncertainties in the fits to the instrument response functions (errors in $\alpha$, see Table 3.2) and the uncertainty in the parameterization of the area coverage (errors in $\delta$ ). For $\delta$ we use the value derived by Fisher et al. (1998), but unfortunately they do not quote an error for $\delta$. Thus we estimate that error by taking the difference of the minimum and maximum slopes from their Fig. 4. Through this we estimate their error in $\delta$ to be 0.2 . For $\beta$ we cannot provide an error, because this is the theoretical expectation for the nanoflare or Alfvén wave heating. Also, we cannot give an error for $\gamma$, because we assume $\gamma=1$.

\subsection{Discussion}

The most important and central result of our study is that the power-law indices, as derived from our simple analytical model, match the observed values well. The values of the power-law indices $m$ from Eq. (3.13) listed in Table 3.2 are generally in the range from about 1 to almost 2. Thus they match the values found in observations (Table 3.1) remarkably well, maybe with the exception of the study by Kochukhov et al. (2020). Based on this, we conclude that our analytical approach, and hence the RTV scaling laws, can capture the processes in stellar coronae qualitatively and quantitatively well.

In the following, we will first discuss the implications of the main result in terms of discriminating different heating mechanisms (Sect. 3.5.1). We will then consider special (limiting) cases of our approach. In particular, we will address the question of whether or not changes of active region size or peak magnetic field strength can alone be responsible for the changes in X-ray emission (Sect. 3.5.2), and what role the spatial structuring of the magnetic field on the surface might play (Sect. 3.5.3).

\subsubsection{Discriminating heating mechanisms}

With our simplified approach, it is hard to distinguish between different heating mechanisms. Mainly, this is because of the large scatter found in the power-law index $m$ for $L_{x} \propto \Phi^{m}$ in Eq. (3.13) introduced by different X-ray instruments. As seen from Table 3.2, $m$ differs by only $20 \%$ to $30 \%$ between the cases of nanoflare $(\beta=2)$ and Alfvén wave heating $(\beta=1)$. However, combining observations from different sources (as necessarily done in data compilations), will imply having different responses of the $\mathrm{X}$-ray emission to the coronal temperature, here quantified by the power-law index $\alpha$ (Sect. 3.2). This can lead to differences in the index $m$ by almost a factor of two (cf. Table 3.2). Consequently, when mixing data from different instruments, the imprints of different heating mechanisms would be swamped by the noise introduced by the different temperature responses.

To distinguish different heating mechanisms, future observational studies would have to carefully evaluate the impact of the temperature response of the instruments used. One could use (a) just one single instrument, (b) show the different instruments in a combined study separately, or (c) use a theoretical approach to normalize the observed X-ray emission of each instrument according to its temperature response.

With all these uncertainties, our analysis would slightly favor nanoflare heating over the Alfvén wave model. The values for the power-law index $m$ we find in Table 3.2 for 
Alfvén waves range from $0.8 \pm 0.2$ to $1.2 \pm 0.3$. As such, they seem to be at the lower end of what is found in observations that show mostly values from just below 1 to below 2 (except for the recent study of Kochukhov et al. 2020, see Table 3.1). Hence, the indices $m$ for nanoflare heating ranging from $1 \pm 0.3$ to $1.6 \pm 0.4$ seem to be a better fit to observational studies.

Considering the uncertainties, the values of $m$ derived by our model largely overlap with the observations. (see Table 3.1). There is the tendency in the observations to show values of $m$ in the upper range of what is predicted by our model (Alfvén wave and nanoflare) and typically the nanoflare model yields larger values of $m$ than the Alfvén wave model. Hence, we consider the nanoflare model to be a slightly better candidate for the stellar X-ray activity than the Alfvén model.

\subsubsection{Magnetic flux and area coverage}

Another key element in our scaling for $L_{x} \propto \Phi^{m}$ in Eq. (3.13) is the relation of magnetic flux and area as parameterized in Eq. (3.7) by $\delta$. While we know from the Sun and solarlike stars that this should be on the order of 0.8 (Fisher et al. 1998; Kochukhov et al. 2020), it is instructive to consider two limiting cases, namely $\delta=0$ and $\delta=1$.

We first consider the case $\delta=1$. According to Eq. (3.7) this implies that the magnetic flux is strictly proportional to the area covered by an active region, $\Phi \propto A$. Hence the (average) magnetic field strength in each active region would be the same, and the magnetic flux would only change by changing the area. Then the expression for the power-law index $m$ for $L_{x} \propto \Phi^{m}$ as given in Eq. (3.13) simplifies to

$$
\delta=1 \quad \longrightarrow \quad m=\frac{1}{7}(4+\alpha) .
$$

Interestingly, in this case there is no dependence on $\beta$ for $S_{z} \propto B^{\beta}$ in Eq. (3.4). Our result does not depend on the actual choice of the heating mechanism. Instead, the relation of the coronal emission to the magnetic flux would only depend on the choice of the instrument through $\alpha$, the wavelength range that is considered (see Eq. 3.3 and Table 3.2). For values of $\alpha$ in the range of 0.7 to 3 (cf. Table 3.2) the values of $m$ would be in the range of 0.7 to 1 . These values fall short of the observations. Thus we conclude that increasing the magnetic flux just by increasing the area $(\delta=1)$ would not provide a sufficiently steep increase of the coronal emission with magnetic flux in $L_{x} \propto \Phi^{m}$.

In the other limiting case, $\delta=0$, the change in the magnetic flux would only be due to the increase in the (average or peak) magnetic field strength. This implies that the magnetic flux in Eq. (3.7) would be independent of the area and we find from Eq. (3.13)

$$
\delta=0 \quad \longrightarrow \quad m=\frac{\beta \gamma}{7}(2 \alpha+8) .
$$

This gives a much steeper dependence of $L_{x} \propto \Phi^{m}$ than for $\delta=1$. Again using $\alpha$ in the range 0.7 to 3 (cf. Table 3.2) we find values of $m$ in the range 1.3 to 4 . Of course, considering the studies of, for example, Fisher et al. (1998) and Kochukhov et al. (2020), a value of $\delta=0$ is unrealistic for the Sun and solar-like stars. However, the steep dependence of the coronal emission $L_{x}$ on the magnetic flux $\Phi$ that we find in this case might help us to understand the high levels of observed X-ray emission of rapidly rotating stars, which 
still show an increase of X-ray activity with increasing rotation (e.g., Pizzolato et al. 2003; Reiners et al. 2014; Wright and Drake 2016). Should the star be (more or less) completely filled with active regions, then the only way to increase the magnetic flux, and therefore its X-ray luminosity, further would be to increase the surface magnetic field strength. Observations of very high average magnetic field strengths on the order of several $1000 \mathrm{G}$ on more active stars (Reiners 2012) indicates that this scenario could be realistic.

\subsubsection{Spatial structure of the magnetic field}

So far, we assumed that the length scale $L$ of the coronal structures, namely the loops, is directly proportional to the linear extent of the active region. Now we explore the effects on the scaling of coronal emission with magnetic flux if the length scale were independent of the active region size.

The assumption that the length scale is given through the active region size is expressed through Eq. (3.8), $L \propto A^{1 / 2}$, and is justified for solar active regions (e.g., Cameron et al. 2010). In general, this does not have to be the case, and stellar observations suggest that large starspots have an internal structure (e.g., Solanki 2002). Thus, it is plausible that generally in (stellar) active regions the distances between opposite magnetic polarities might not be related to the active region size. Consequently, Eq. (3.8) would no longer hold. To explore an extreme case, in the following we assume that loop length $L$ would be independent of the area, and in particular assume that $L$ would be a constant. For example, one might argue that for an active star the size of the coronal structures we see might be related to the coronal pressure scale height.

Assuming a constant loop length $L$, therefore not considering Eq. (3.8), we can repeat the derivation of Eq. (3.13) for the scaling between coronal emission and magnetic flux, $L_{x} \propto \Phi^{m}$. Then we find for the power-law index

$$
\begin{aligned}
& \text { constant } \\
& \text { loop length: }
\end{aligned} \longrightarrow\left\{\begin{array}{r}
m=\delta+p(1-\delta) \\
\text { with } p=\frac{\beta \gamma}{7}(2 \alpha+8) .
\end{array}\right.
$$

As expected, for $\delta=0$ this gives the same result as discussed above with Eq. (3.15). For $\delta=1$ we find that coronal emission is strictly linear with the magnetic flux. Quantitatively, this is similar to the result above with Eq. (3.14), where we found $m$ to be a bit smaller but close to unity.

For the case of the Sun, it is well established that the total magnetic flux (integrated over the whole solar surface) during the maximum activity is mostly increasing through the number of active regions and not by increasing their size (e.g., Tang et al. 1984). Hence, we can also expect the length of coronal loops on the Sun not to change (significantly) with activity level. Therefore, Eq. (3.16) might be the appropriate description for the relation of X-ray emission to magnetic field for the Sun and its cycle.

In general, the values for the power-law index $m$ found here in Eq. (3.16) are quantitatively similar to the values when not assuming constant loop length as given through Eq. (3.13). The values of $m$ listed in Table 3.2 would change typically only by about $20 \%$. This shows that within the limitations of our analytical approach for the scaling laws, the loop length does not have a significant impact. 
Still, numerical models of active regions will be needed to investigate the applicability of our simplified analytical approach. For example, if the size of the active region is increased, the total magnetic energy of the volume associated with the active region will also increase. This increase can be expected to be steeper than proportional to the magnetic flux at the surface. This is similar to increasing the separation of opposite polarities in a magnetic dipole. The work done to separate the two poles (like separating two magnets) goes into magnetic energy stored in the volume, even though the magnetic flux at the surface stays the same. While we find a good match between our simple model and observations, future numerical models will have to show if the basics of the analytical considerations presented here will hold.

\subsubsection{X-ray emission for rapid rotators}

For rapidly rotating stars the coronal X-ray emission becomes independent of their rotation rate (see, e.g., Pizzolato et al. 2003). Sometimes this is called the saturation regime, but it remains unclear what causes this behavior (e.g., Reiners et al. 2014). Assuming that in this regime also the surface magnetic flux is increasing with increasing rotation rate, our model would have to predict that the X-ray emission does not change with magnetic flux. Consequently, $m$ in Eq. (3.13) would have to vanish.

So, to test if our model is applicable in this saturation regime, we simply set $m=0$ in Eq. (3.13). As before, we assume that $\gamma=1$, meaning that the heating rate is proportional to the Poynting flux (cf. Eq. (3.5)). With this we can solve for $\delta$,

$$
\delta=\frac{2 \beta}{2 \beta-1}
$$

The result does not depend on $\alpha$, meaning that in this regime it would not matter which instrument or filter was used for the diagnostics.

Interestingly, for both types of our heating model we find that $\delta>1$. More precisely, for the nanoflare model $(\beta=2)$ we get $\delta=1.33$ and for the Alfvén model $(\beta=1)$ we get $\delta=2$. This would imply that an increase in the total surface magnetic flux would lead to a decrease in the magnetic active area, meaning that the magnetic flux would concentrate in smaller and smaller regions. Such peculiar behavior would require an additional effect to operate that needs to overcome the strong magnetic pressure forces. However, this seems rather unphysical, and to our knowledge is without observational support.

Overall, we can conclude that the X-ray emission of very active rapidly rotating stars is not governed by the same relations as for solar-like stars. Not surprisingly, our model is not suitable to describe the stellar X-ray emission in that specific regime.

\subsection{Conclusions}

We derived an analytical scaling relation of the coronal X-ray emission with the unsigned surface magnetic flux, $L_{x} \propto \Phi^{m}$ in Eq. (3.13). Previously, this relation had only been derived using observations, without the backing of a theoretical framework. We based our approach on the coronal loop scaling laws of Rosner et al. (1978) (see Eq. (3.9) and 
(3.10)), and the idea that the heating of the corona is mainly driven by an upward-directed Poynting flux generated in the photosphere.

The power-law index $m$ that we derive in Eq. (3.13) depends on the area of the active region, the heating mechanism, and the wavelength range covered by the respective $\mathrm{X}$ ray instrument, namely, its temperature response function. Each of these factors can be represented by power laws. The active region area impact is constrained observationally $(\delta=0.819$, Eq. 3.7), the heating mechanism is inspired by basic considerations ( $\beta$ from 1 to 2; Eq. 3.4), and the temperature response between 1 and $10 \mathrm{MK}$ is based on atomic data ( $\alpha$ in the range of 1 to 3, Table 3.2).

The power-law indices $m$ we find through our analytical approach are generally in a range between just below $m \approx 1$ and almost 2 (see Table 3.2). This is within the range found by most observations, which are mostly composed of a combination of stellar studies with different instruments (see Table 3.1; a larger value only found by Kochukhov et al. 2020). As such, we consider our simple analytical model approach to be a good first step to build a theoretical foundation for the observed power-law relations between $\mathrm{X}$-ray emission and magnetic field. However, with our simplified model approach it is difficult to distinguish between different heating mechanisms, mainly because the different $\mathrm{X}$-ray instruments have quite different responses to the temperature of the coronal plasma. 



\section{Model setup}

\subsection{Introduction}

The numerical setup, presented in this chapter, models a small part of the solar corona above an active region in a 3D Cartesian box (see Bingert 2009, for more details). The model extends in the vertical direction up to the corona, including also the photosphere. The motivation of this model is the fieldline braiding mechanism proposed by Parker (1972, 1983). At the lower boundary, we include the vertical magnetic field of an active region together with a description of photospheric velocities mimicking solar granulation. The shuffling of the footpoints of the photospheric magnetic fieldlines because of the horizontal velocities will generate the necessary energy flux (or Poynting flux) needed to heat the corona to high temperatures of several million Kelvin degrees, similar to the solar corona. Once the system reaches an equilibrium state, the energy input in the corona is balanced by the radiative losses. In this state, the modeled corona can maintain a high temperature indefinitely.

Our computational box is divided into four parts. The lower part of our computational domain is the photosphere, where the active region is located. In the photosphere, the shuffling of the magnetic fieldlines will induce currents in the corona that will dissipate and heat the corona. The chromosphere is the part of the box located above the photosphere. Given the highly complicated nature of the chromosphere, it is only treated as a reservoir for energy. We mainly care about the energy transfer in the corona. Therefore, excluding the chromosphere from our analysis will not affect the results. The transition region is a thin layer located between the chromosphere and the corona. In the transition region, the temperature increases steeply, reaching from a few thousand Kelvins to coronal temperatures of one million Kelvin. The high gradients in temperature have to be resolved numerically so that numerical instabilities are avoided and will not affect the simulations. The corona extends for the largest part of our numerical box. For the proper physical description of the corona, we include an optically thin radiative loss function and a Spitzer heat conduction along the magnetic field. This model has been proved successful in describing some of the characteristics observed in the solar corona (see e.g. Bingert and Peter 2011; Warnecke and Peter 2019a). Consequently, it can be used for a parameter study and extend the analysis even to other stars.

We aim to run several simulations to test the effect of the surface magnetic flux on the coronal X-ray emission. The reason for that is to explain the observed X-ray emission coming from stellar coronae of stars more active than the Sun. To be able to run several simulations for our analysis, we choose a spatial resolution of $390 \mathrm{~km}$.

Our work is divided into two parts. For the first part, we increase the surface magnetic 
flux by increasing the strength of the vertical surface magnetic field of the active region. In this case, we keep the box size fixed with a volume of $50 \times 50 \times 50 \mathrm{Mm}^{3}$. For the simulations we used the supercomputers in Gesellschaft für wissenschaftliche Datenverarbeitung mbH Göttingen (GWDG) with a $128^{3}$ grid points in all directions. The results will be presented in Chap. 5. For the second part, we increase the surface magnetic flux by increasing the surface area covered by the active region. In this case, our numerical box has to increase. The biggest computational box reaches a volume of $200 \times 200 \times 200$ $\mathrm{Mm}^{3}$. The individual active regions have the same surface vertical magnetic field strength fixed at a constant value. The simulations have been carried out at the Max Planck Computing and Data Facility (MPCDF) in Münich. The number of grid points scales with the size, but the spatial resolution is constant at $390 \mathrm{~km}$. The results of this work will be presented in Chap.6. Finally, the different physical parameters have the same values for all simulations so that the corona will be affected solely by the change in the surface magnetic flux.

\subsection{Numerical model}

The simulations were conducted with the Pencil Code $^{1}$ (Brandenburg et al. 2020). The Pencil Code solves the 3D MHD equations spanning from the photosphere up to the corona numerically. In this section, we are going to summarise the equations as they are implemented in the code.

\subsubsection{Equations}

The code solves the partial differential equations of mass, momentum, energy, and the induction equation, which are coupled together. The equations implemented in the code have the general form,

$$
\frac{\partial \boldsymbol{\Phi}}{\partial t}=f(\boldsymbol{\Phi}) .
$$

Where $\boldsymbol{\Phi}$ represents the different quantities that need to be integrated. The left-hand side represents the time derivative, whereas the function $f(\boldsymbol{\Phi})$ in the right-hand side represents spatial partial derivatives. For the time integration, a third-order Runge-Kutta scheme is used and for the spatial derivatives, a 6th order finite difference numerical scheme is used. In total, there are eight partial differential equations which are summarised below. We start with the continuity equation expressed in the form,

$$
\frac{\partial \ln \rho}{\partial t}=-\nabla \cdot \boldsymbol{u}-(\boldsymbol{u} \cdot \nabla) \ln \rho .
$$

Where $\ln \rho$ is the plasma density and $\boldsymbol{u}$ is the plasma velocity. To smear out any instability in the plasma density, we include a mass diffusion term which is also added in the continuity, momentum, and energy equation.

\footnotetext{
${ }^{1}$ https://github.com/pencil-code/
} 
To describe the plasma motion, it is necessary to solve the momentum equation,

$$
\frac{\partial \boldsymbol{u}}{\partial t}=-(\boldsymbol{u} \cdot \nabla) \boldsymbol{u}+\zeta(\nabla \nabla \cdot \boldsymbol{u})+\frac{1}{\rho}[-\nabla p+\boldsymbol{j} \times \boldsymbol{B}-\rho \nabla \Phi+2 v \nabla \circ(\rho \underline{S})] .
$$

The forces included in our model are the pressure gradient with $p$ being the pressure, the Lorentz force with $\boldsymbol{j}$ and $\boldsymbol{B}$ being the current density and magnetic field, the gravitational force with a gravitational potential $\Phi$ and the viscous force with $\underline{S}$ being the rate of the strain tensor,

$$
\underline{S}=\frac{1}{2}\left(\frac{\partial u_{i}}{\partial x_{j}}+\frac{\partial u_{j}}{\partial x_{i}}-\frac{2}{3} \delta_{i j} \boldsymbol{\nabla} \cdot \boldsymbol{u}\right)
$$

The value of $v$ in the solar corona, according to transport theory, is on the order of $v=10^{10}$ $\mathrm{m}^{2} / \mathrm{s}$. For that reason, we set the kinematic viscosity to $v=10^{10} \mathrm{~m}^{2} / \mathrm{s}$ in the whole computational box. Finally, it is possible for the plasma at different parts of the numerical box to reach a Mach number greater than unity. The Mach number is defined as the ratio of the velocity $u$ to the sound speed $c_{s}, M=u / c_{s}$. For $M>1$, the plasma velocity is hypersonic, and shocks will be created. To properly resolve any instabilities created by shocks, we include a shock viscosity term $\zeta(\nabla \nabla \cdot \boldsymbol{u})$, with the bulk viscosity coefficient set to $\zeta=10^{8} \mathrm{~m}^{2} / \mathrm{s}$.

The equation describing the change of energy in the numerical box is the energy equation,

$$
\begin{aligned}
\frac{\partial \ln T}{\partial t} & =-(\boldsymbol{u} \cdot \nabla) \ln T-(\gamma-1) \nabla \cdot \boldsymbol{u}+\eta \mu_{0} \dot{j}^{2}+2 \rho v \underline{S}^{2} \\
& +\frac{\gamma}{c_{p} \rho T}\left[-\left(\frac{0.8 \rho}{m_{p}}\right)^{2} Q(T)+\nabla \cdot\left(K_{0} T^{5 / 2} \boldsymbol{b}(\boldsymbol{b} \cdot \nabla T)\right)\right. \\
& \left.+\nabla \cdot\left(c_{p} \chi \rho \nabla T\right)+\nabla \cdot(c|\nabla T| \nabla T)\right] .
\end{aligned}
$$

Here $\ln T$ is the temperature. The constant $\gamma$ is the adiabatic coefficient for the ideal gas with $\gamma=5 / 3, c_{p}$ is the heat capacity at constant pressure, and $m_{p}$ is the proton mass. The magnetic resistivity $\eta$ follows a specific profile with the coronal value set to $\eta=10^{10} \mathrm{~m}^{2} / \mathrm{s}$. For the lower part of the box, $\eta$ has a lower value. The implications of the resistivity will be discussed later. The function $Q(T)$ is the radiative loss function. Finally, the coefficients $K_{0}, \chi$, and $c$ are the Spitzer constant, the heat diffusion constant, and the isotropic heat conduction coefficient respectively. Notice here that we include three different heat conduction terms. The first term is the dominant Spitzer term $\nabla$. $\left(K_{0} T^{5 / 2} \boldsymbol{b}(\boldsymbol{b} \cdot \nabla T)\right)$ which transfers heat along the magnetic fieldlines (see Sect. 2.2.4.3). The second term is the heat conduction $\nabla \cdot\left(c_{p} \chi \rho \nabla T\right)$ which is most efficient at the lower part of the box. Finally, we also include an isotropic heat conduction term $\nabla \cdot(c|\nabla T| \nabla T)$ to stabilize the code and resolve the steep gradients of temperature that might arise at different parts of the numerical box. The last equations to close the system is the induction equation,

$$
\frac{\partial \boldsymbol{A}}{\partial t}=\boldsymbol{u} \times(\nabla \times \boldsymbol{A})+\nabla(\eta \nabla \cdot \boldsymbol{A})
$$


where $\boldsymbol{A}$ is the vector potential. Here we use the resistive gauge $\phi=\eta \boldsymbol{\nabla} \cdot \boldsymbol{A}$. For numerical reasons, we also include hyperviscosity terms in the induction equation.

One of the main problems in our simulations is to determine the magnetic resistivity $\eta$ properly. For the coronal part, the value of the resistivity is set to a constant value of $\eta=5 \cdot 10^{10} \mathrm{~m}^{2} / \mathrm{s}$. This value is much larger than typical values proposed from transport theory (typical values yield $\eta=1 \mathrm{~m}^{2} / \mathrm{s}$ ). The choice of this value is to ensure that the grid Reynolds number will be in the order of unity,

$$
R_{e m}^{\text {grid }}=\frac{u_{\max } \delta x}{\eta} \simeq 1 .
$$

For a grid resolution of $\delta x=390 \mathrm{~km}$ and a typical velocity of $u_{\max }=150 \mathrm{~km} / \mathrm{s}$, yields a resistivity of $10^{10} \mathrm{~m}^{2} / \mathrm{s}$. The magnetic Reynolds number compares the time-scale of diffusion to advection. If we set the typical coronal magnetic resistivity of $\eta=1 \mathrm{~m}^{2} / \mathrm{s}$ in our model we get $R_{e m}^{\text {grid }}>>1$. That would mean that the plasma is mainly advected, and we would not have any diffusion and thus no heating. The value of $\eta$ chosen in our model ensures diffusion of the magnetic field and heating of the corona.

Keeping the high constant value of resistivity in the whole box combined with the random photospheric velocities will lead to fast diffusion of the surface magnetic field after a short period. The magnetic flux in the Sun is created deep in the convection zone and then emerges in the photosphere. Because the roots of the magnetic fieldlines lie underneath the solar surface, the active regions are stable for long periods of many days. However, the process of flux emergence is not included in our model. To avoid large diffusion of the surface magnetic field, we lower the magnetic resistivity at the three lowest grid points.

\subsubsection{Spatial derivatives}

For the calculations of spatial derivatives, we employ a 6th order finite difference numerical scheme. A general expression of the first and second derivative is (see Pencil code guide manual, Brandenburg 2018),

$$
\begin{aligned}
f_{i}^{\prime} & =\left(-f_{i-3}+9 f_{i-2}-45 f_{i-1}-9 f_{i+2}+f_{i+3}\right) /(60 \delta x) \\
f_{i}^{\prime \prime} & =\left(2 f_{i-3}-27 f_{i-2}+270 f_{i-1}-490 f_{i}+270 f_{i+1}-27 f_{i+2}+2 f_{i+3}\right) /\left(180 \delta x^{2}\right),
\end{aligned}
$$

where $i$ denotes the point of interest to calculate the derivative, and $\delta x$ denotes the grid space. The 6th order scheme means that for calculating the derivative at the position $x_{0}$ requires the knowledge of six extra points around that specific point, three in each direction. This particular numerical scheme is highly accurate, efficient, and the memory usage is minimal. Employing higher-order schemes will increase the accuracy, but the computation time will increase as well. Therefore performing a large number of simulations will be challenging.

\subsubsection{Time step constraints}

The time evolution of the variables is calculated through a third-order Runge-Kutta scheme. The Runge-Kutta method is used to solve general differential equations of the form, 


$$
\frac{d y_{i}}{d t}=F_{i}\left(y_{k}, t\right)
$$

Knowing the $y_{i}$ at a specific time $t_{0}$ we can calculate the $y_{i}$ at $t_{0}+\delta t$ by evaluating $F_{i}$. The Runge-Kutta method follows this procedure in multiple sub-steps. The third-order RungeKutta used in our code solves the time evolution problem by employing three sub-steps for one time step, hence the name third order. The solution will have the form,

\begin{tabular}{c|c|c}
$\mathrm{t}$ & $\mathrm{y}$ & \\
\hline$t_{0}$ & $y_{0}$ & $k_{1}=\delta t F\left(y_{0}, t_{0}\right)$ \\
$t_{1}=t_{0}+\frac{8}{15} \delta t$ & $y_{1}=y_{0}+\frac{8}{15} k_{1}$ & $k_{2}=\delta t F\left(y_{1}, t_{1}\right)$ \\
$t_{2}=t_{1}+\frac{2}{3} \delta t$ & $y_{2}=y_{0}+\frac{1}{4} k_{1}+\frac{5}{12} k_{2}$ & $k_{3}=\delta t F\left(y_{2}, t_{2}\right)$ \\
\hline$t=t_{0}+\delta t$ & $y=y_{0}+\frac{1}{4} k_{1}+0 k_{2}+\frac{3}{4} k_{3}+O\left(\delta t^{4}\right)$ &
\end{tabular}

This method, in general, requires less memory than other similar schemes, thus, making our code much more time-efficient.

The time step will generally be defined by the Courant time step given by,

$$
\delta t=\min \left(c_{\delta t} \frac{\delta x_{\min }}{U_{\max }}, c_{\delta t, v} \frac{\delta x_{\min }^{2}}{D_{\max }}, c_{\delta t, s} \frac{1}{H_{\max }}\right)
$$

where $c_{\delta t}, c_{\delta t, v}, c_{\delta t, s}$ are the Courant-Friedrichs-Lewy (CFL) coefficients. The CFL coefficients are smaller than unity, and they are generally used for convergence while solving differential equations numerically. $\delta x_{\text {min }}$ defines the minimum grid space in the numerical box and $U_{\max }$ denotes the maximum velocity in our box as,

$$
U_{\max }=\max \left(u+\sqrt{c_{s}^{2}+u_{A}^{2}}\right),
$$

where $c_{s}$ is the sound speed and $u_{A}$ is the Alfvén speed. The maximum velocity in our box will be either the advective velocity, the sound speed, or the Alfvén velocity. Furthermore, $D_{\max }$ denotes the maximum diffusivity,

$$
D_{\max }=\max (v, \gamma \chi, \eta)
$$

where $v$ is the kinematic viscosity, $\chi$ is the thermal diffusivity, and $\eta$ is the magnetic resistivity. Finally, the $H_{\max }$ represents the right-hand side of the energy equation. However, since our simulations are dominated by thermal diffusivity, this term will not be significant. In conclusion, the time step will be constrained by the thermal diffusivity, limiting the computational speed significantly. To boost the speed, we have to employ various techniques discussed next.

\subsubsection{Non-Fourier treatment of heat flux}

The efficiency of the Spitzer heat conduction puts constraints on the time step. To increase the time step and speed up our simulations, we use a non-Fourier description of the heat flux (Warnecke and Bingert 2020),

$$
\frac{\partial \boldsymbol{q}}{\partial t}=-\frac{1}{\tau_{\text {Spitzer }}}(\boldsymbol{q}+K \nabla T),
$$


where $\tau_{\text {Spitzer }}$ is the heat flux relaxation time, and $K$ is the Spitzer tensor with $K=$ $K_{0} T^{5 / 2} \hat{b} \hat{b}$ operating along the magnetic fieldlines. Here $\hat{b}$ is the unit vector along the magnetic fieldlines. From Eq. (4.13) we solve for $\boldsymbol{q}$ and then this is substituted in Eq. (4.5). With this approach we replace the Spitzer time step $\delta t_{\text {Spitzer }}=\delta x^{2} / \chi_{\text {Spitzer }}$ with two new time steps,

$$
\delta t_{1}=\delta x \sqrt{\left(\frac{\tau_{\text {Spitzer }}}{\chi_{\text {Spitzer }}}\right)}=\frac{\delta x}{c_{\text {Spitzer }}}, \quad \text { and } \quad \delta t_{2}=\tau_{\text {Spitzer }} .
$$

Here $\delta x$ is the grid spacing and $\chi_{\text {Spitzer }}=|\boldsymbol{K}| / \rho c_{V}$ is the diffusivity of the Spitzer heat conduction. The Eq. (4.13) can be expressed as a wave equation, therefore the $\delta t_{1}$ is also related to the wave propagation speed $c_{\text {Spitzer }}$. Notice here that $\delta t_{1}$ has a linear dependence on the grid size $\delta x$, which leads to a substantial increase of the computational time as the spatial resolution is increased.

Even though the $\tau_{\text {Spitzer }}$ can be adjusted manually, it is preferable to use the automatic adjustment implemented in the code by Warnecke and Bingert (2020). We first set the time step of the heat conduction to be the dominant, i.e. the smallest. The next dominant time scale in the box will be the Alfvén crossing time $\delta t_{A}=\delta x / u_{A}$ with $u_{A} \propto B / \sqrt{\rho}$ the Alfvén speed. The idea is that the $\tau_{\text {Spitzer }}$ is limited by the Alfvén time scale and we set,

$$
c_{\text {Spitzer }}=\sqrt{2} u_{A}, \rightarrow \tau_{\text {Spitzer }}=\frac{\chi_{\text {Spitzer }}}{2 u_{A}^{2}} .
$$

One problem that arises here is that if $\tau_{\text {Spitzer }}$ evolves freely, then it might get too large. In that case, the Spitzer heat conduction will not be dominant anymore and might lead to non-physical solutions. For that reason, we set an upper limit to the time step $\tau_{\text {Spitzer }}$. In addition, the Spitzer coefficient $\chi_{\text {Spitzer }}$ reduces to low values in parts of low temperature and high density in the chromosphere or transition region. Therefore, this will reduce $\tau_{\text {Spitzer }}$ in those regions. Even though the Spitzer heat conduction is not dominating in those lower parts, the reduced $\tau_{\text {Spitzer }}$ will decrease the overall time step tremendously. To avoid that problem, we set a lower limit of the time step to be in between of the Alfvén, and the advective time step,

$$
\min \left(\delta t_{A}, \frac{\delta x}{\sqrt{c_{s}^{2}+u^{2}}}\right) \leq \tau_{\text {Spitzer }} \leq \tau_{\text {Spitzer }, 0}
$$

where $c_{s}$ is the sound speed, $u$ is the advective velocity and we set $\tau_{\text {Spitzer }, 0}=100 \mathrm{~s}$. Using the non-Fourier treatment of the heat flux Warnecke and Peter (2019b) report a boost in the time step by a factor of two.

\subsubsection{Semi-relativistic Boris correction}

The Alfvén speed approaches the speed of light in regions of high magnetic field strength and low density, i.e. above active regions (Rempel 2017). As a result, two main problems arise. First of all, the MHD approximation of neglecting the displacement current for non-relativistic velocities discussed in Chap. 2 is no longer valid. In addition, a significant increase of the Alfvén speed will lead to a very small time step. For those reasons, it is implemented in the code a semi-relativistic correction to the Lorentz force (Boris 1970; Gombosi et al. 2002). The Lorentz force in this framework is replaced by, 


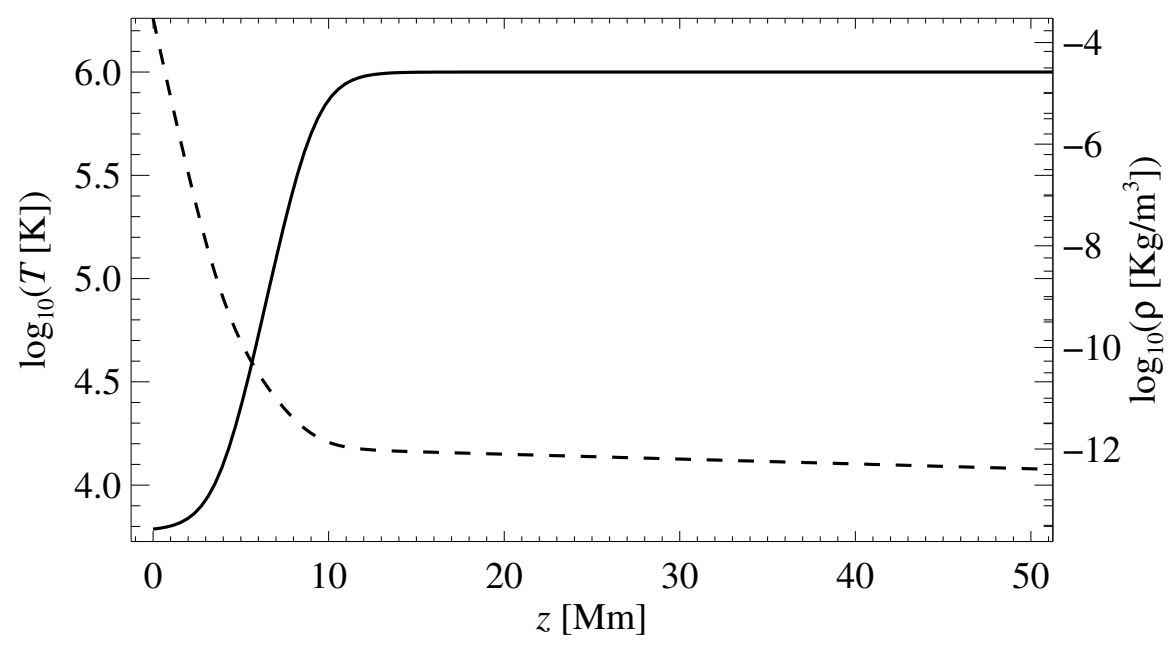

Figure 4.1: Initial temperature and density profile implemented in our numerical experiments. Solid line show the initial temperature profile. Dashed line shows the initial density stratification based on hydrostatic equilibrium.

$$
\frac{\boldsymbol{j} \times \boldsymbol{B}}{\rho} \rightarrow \gamma_{A}^{2} \frac{\boldsymbol{j} \times \boldsymbol{B}}{\rho}+\left(1-\gamma_{A}^{2}\right)\left(\boldsymbol{I}-\gamma_{A}^{2} \frac{\boldsymbol{B} \boldsymbol{B}}{\boldsymbol{B}^{2}}\right)\left(\boldsymbol{u} \cdot \nabla \boldsymbol{u}+\frac{\nabla p}{\rho}-\boldsymbol{g}\right)
$$

where $\gamma_{A}^{2}=1 /\left(1+u_{A}^{2} / c^{2}\right)$ is the correction factor similar to the Lorentz factor in the special relativity. For the non-relativistic limit, the factor $\gamma_{A} \simeq 1$, and we retrieve the usual Lorentz force. For relativistic velocities, $u_{A} \simeq c$ the above correction reduces the Lorentz force. The definition of the unit vector along the magnetic fiedlines $\hat{b}=\boldsymbol{B} /|\boldsymbol{B}|$ explains the factor $\boldsymbol{B} \boldsymbol{B} / \boldsymbol{B}^{2}$ in Eq. (4.17). This method can also be used to increase the time step and prevent a crash of our simulations. The details of this method are discussed in Warnecke and Bingert (2020) and the implementation in the Pencil Code are discussed in Chatterjee (2018). The idea is to reduce the speed of light in the regions where the Alfvén speed is large. Following the work of Rempel (2017) the Alfvén time step will be,

$$
d t_{A} \rightarrow d t_{A} \sqrt{1+\left(\frac{u_{A}^{2}}{c_{A}^{2}}\right)^{2}}
$$

where $c_{A}$ is the limited speed of light. In our simulations we choose $c_{A}=10000 \mathrm{~km} / \mathrm{s}$ which leads to a time step of $d t_{A} \simeq 20 \mathrm{~ms}$. With this method, we ensure that the Alfvén time step will have a lower limit and it will not affect the overall time step in our simulations. As discussed in Warnecke and Peter (2019b), a combination of the non-Fourier heat flux scheme and the Boris correction leads to an increase of the time step by a factor of 10 or even a factor of 30 . In conclusion, the Boris correction and the non-Fourier treatment of the heat flux combined will significantly boost the computational speed of our simulations. This allows performing a large number of simulations for a parameter study possible. 


\subsection{Initial conditions}

Initially, we set a specific stratification profile for temperature $T$ and density $\rho$ in the computational box. The specific profile for $T, \rho$ are depicted in Fig. 4.1. The temperature profile has the form,

$$
T=\left(T_{c}-T_{p}\right) \cdot\left(\frac{1}{2} \cdot \tanh \left(\frac{z-z_{p}}{z_{c}}\right)+\frac{1}{2}\right)+T_{p}
$$

The photospheric temperature is set to $T_{p}=6000 \mathrm{~K}$, and it rises after the transition region to $T_{c}=10^{6} \mathrm{~K}$. The values $z_{p}, z_{c}$ determine the height of the corona. The initial density, on the other hand, is determined by hydrostatic equilibrium,

$$
\nabla p=-\rho g
$$

where the gravity is set to the solar value with a constant gravitational acceleration $\boldsymbol{g}=$ $(0,0,-g)$ with $g=274 \mathrm{~m} / \mathrm{s}^{2}$, and the pressure is,

$$
p=\frac{(\gamma-1)}{\gamma} c_{p} \rho T
$$

The density depicted in Fig. 4.1 (dashed line) drops from $3 \cdot 10^{-4} \mathrm{~kg} / \mathrm{m}^{3}$ to $10^{-12} \mathrm{~kg} / \mathrm{m}^{3}$ in the corona.

Before starting the simulations, we also provide the vertical magnetic field at the bottom boundary of the numerical box. Previous studies such as Gudiksen and Nordlund (2005b,a), and Bingert and Peter (2011) used a scaled-down active region that was provided by an MDI image of the Sun. However, in our study, we employ an actual magnetogram of the active region, AR 11102 observed on August 30th, 2010 by Helioseismic and Magnetic Imager (HMI;Schou et al. (2012)). The size of this active region was $164 \times 164 \mathrm{Mm}^{2}$, and it had a surface magnetic flux on the order of $\Phi \simeq 8 \times 10^{21} \mathrm{Mx}$. The size and amount of surface magnetic flux of AR 11102 are typical for solar active regions. For the purpose of our studies the original size of the active regions is scaled accordingly to fit our computational box. In one specific simulation discussed as an example in this chapter, the active region is shown in Fig. 4.2 and the computational domain is scaled, so that it covers an area of $200 \times 200 \mathrm{Mm}^{2}$. A potential field extrapolation is then performed to fill the box with a magnetic field. The size of the active region will be changed in Chap. 6 so that to increase or decrease the total surface magnetic flux. The reason is to study how the total magnetic flux is related to the total coronal X-ray emission as it is observed in stellar studies.

\subsection{Potential field extrapolation}

We initialize the magnetic field in the box via a potential field extrapolation method. The initial vertical magnetogram we impose at the bottom boundary will be the basis for the extrapolation. We assume a current-free field which reduces the Maxwell equations for a potential field to, 


$$
\begin{aligned}
\nabla \times \boldsymbol{B} & =0 \\
\nabla \cdot \boldsymbol{B} & =0 .
\end{aligned}
$$

The previous equations can be solved in Fourier space. The Fourier transform of the magnetic field reads,

$$
\hat{B}_{i}\left(k_{x}, k_{y}\right)=\int_{-\infty}^{\infty} \int_{-\infty}^{\infty} B_{i}(x, y, z=0) e^{-i k_{x} \cdot x-i k_{y} \cdot y} d x d y,
$$

where $i=(x, y, z)$, and we assume an exponentially decaying magnetic field with height $z$ so that $\hat{B}_{i}\left(k_{x}, k_{y}, z\right)=\hat{B}_{i}\left(k_{x}, k_{y}\right) e^{-k z}$ with $k=\sqrt{k_{x}^{2}+k_{y}^{2}}$. The magnetic field at $z=0$ is known from the magnetogram used at the bottom boundary. Using the Fourier transforms, Eq. (4.22) is expressed as,

$$
\begin{array}{r}
i k_{y} \hat{B}_{z}-\partial_{z} \hat{B}_{y}=0 \\
\partial_{z} \hat{B}_{x}-i k_{x} \hat{B}_{z}=0 \\
i k_{x} \hat{B}_{y}-i k_{y} \hat{B}_{x}=0 \\
k_{x} \hat{B}_{x}+k_{y} \hat{B}_{y}+\partial_{z} \hat{B}_{z}=0
\end{array}
$$

where we substituted the Fourier transform of the spatial derivatives $\partial_{x}=i k_{x}, \partial_{y}=i k_{y}$. Solving the above linear set of equations we get,

$$
\begin{aligned}
& \hat{B}_{x}=-i \frac{k_{x}}{k} \hat{B}_{z} \\
& \hat{B}_{y}=-i \frac{k_{y}}{k} \hat{B}_{z} .
\end{aligned}
$$

The vector potential is given by $\boldsymbol{B}=\nabla \times \boldsymbol{A}$, which in Fourier space is expressed as,

$$
\begin{aligned}
& \hat{B_{x}}=i k_{y} \hat{A_{z}}+k \hat{A_{y}} \\
& \hat{B_{y}}=-k \hat{A_{x}}-i k_{x} \hat{A_{z}} \\
& \hat{B}_{z}=i k_{x} \hat{A_{y}}-i k_{y} \hat{A_{x}} .
\end{aligned}
$$

Solving for the vector potential coefficients we get,

$$
\begin{aligned}
& \hat{A_{x}}=i \frac{k_{y}}{k^{2}} \hat{B}_{z} \\
& \hat{A_{y}}=-i \frac{k_{x}}{k^{2}} \hat{B}_{z} \\
& \hat{A_{z}}=0
\end{aligned}
$$

By specifying the vertical magnetic field component at the bottom boundary (i.e. $z=0$ ) through the magnetogram, the Fourier coefficients $\hat{B}_{x}, \hat{B}_{y}, \hat{B}_{z}$ can be calculated and from Eq. (4.23) the magnetic field can be extrapolated in the whole box. This procedure is only done at the initial stage of the simulation. After the start of the simulation, the magnetic field is calculated through the induction equation (see Eq. (4.6)). 

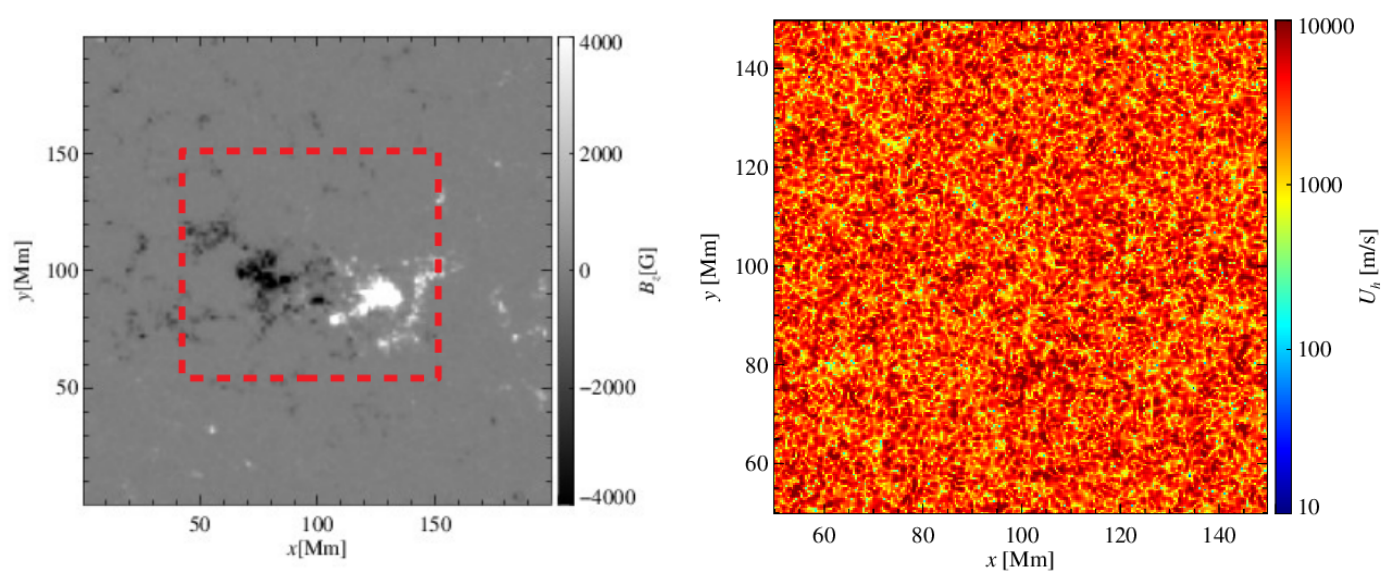

Figure 4.2: Initial $B_{z}$ magnetic field of the active region and photospheric velocities. Left: This particular magnetogram was observed by Helioseismic and Magnetic Imager (HMI) (Schou et al. 2012) onboard of Solar Dynamics Observatory (SDO) on August 30th, 2010 and is used to drive the simulations. Right: Horizontal photospheric velocities $U_{h}$ prescribed in the bottom boundary. The red rectangular box corresponds to the zoomed area of $100 \times 100 \mathrm{Mm}^{2}$ chosen to show the horizontal velocities.

\subsection{Boundary conditions}

All the variables in the horizontal plane (i.e. $x-y$ plane) are periodic. This allows the magnetic fieldlines to connect outside the computational domain. To simulate a roughly bipolar region, where the magnetic fieldlines mainly connect the two magnetic polarities, we include a large enough region of quiet Sun as it is shown in Fig. 4.2.

For the top boundary of the computational box, we make sure that all velocity components are zero. That ensures that there is no plasma escaping out of the box. Furthermore, we make the temperature gradient and the heat flux vanish. That means that no heat flux from the top boundary will affect the coronal heating and dynamics. The density gradients are set to zero as well. Finally, the magnetic field at the top boundary is potential.

On the other hand, for the bottom boundary, we have fixed values of temperature and density. Moreover, horizontal velocities have zero gradients, and the vertical velocity satisfies the divergence-free condition $\nabla \cdot \boldsymbol{u}=0$. The method of prescribing the photospheric velocities similar to the solar granular motion will be discussed below. Finally, the magnetic field is potential, and we also employ a time-dependent update which we will discuss in detail later.

\subsubsection{Photospheric driver}

One important feature missing from our model is the photospheric magneto-convective motions. Since we do not include the convection zone in our simulations, we have to prescribe photospheric velocities similar to the solar granulation motion. The details for the implementation of the velocity driver in the code are discussed in the Ph.D. thesis of Bingert (2009). Here we briefly discuss the main idea of this approach.

The solar photosphere consists of numerous granular structures which transport energy from the convection zone to the surface. After the energy is released, they plunge 
into the convection zone again. This whole process is repeated indefinitely. The granules differ in size from $1 \mathrm{Mm}$ for typical granules to 20-30 Mm for supergranules, and they have typical velocities of roughly $2 \mathrm{~km} / \mathrm{s}$.

A magnetogram of an active region is used at the bottom boundary, therefore, the convection zone has been excluded from the model. That means no granulation can appear self consistently but has to be a priori implemented to shuffle the footpoints and induce currents according to the fieldline braiding mechanism. This method is based on the theory of Voronoi tessellation as discussed in Schrijver et al. (1997) and Gudiksen and Nordlund (2005a). The theory of Voronoi tessellation provides a way to split a $2 \mathrm{D}$ plane into tiles that represent the granules by placing them randomly. Each of these granules will have a weight $w_{i}$ which controls the size of the granules at position $\boldsymbol{x}_{\boldsymbol{i}}$. The horizontal photospheric velocities are depicted in Fig. 4.2 in one of our models. In this figure, we zoomed to an area of $100 \times 100 \mathrm{Mm}^{2}$ for the granules to be visible. The granules created by this process will have three different sizes with a radius of $0.8,1.6,3.2 \mathrm{Mm}$ respectively. A typical solar granule has a typical lifetime of a few minutes. For that reason, the granules in our model are time-dependent, with a lifetime ranging from 5 minutes for the smallsized granules to 80 minutes for the large-sized granules.

In conclusion, this method implemented by Bingert (2009) gives a power spectrum of the horizontal velocities in good agreement with solar observations. The modeled corona captures all the basic properties of the observed solar corona, thus we can consider this approach a good representation of the granular magneto-convective motion.

\subsubsection{Photospheric magnetic field update}

After extrapolating the initial magnetic field, the code will calculate the time evolution through the induction equation,

$$
\frac{\partial \boldsymbol{A}}{\partial t}=\boldsymbol{u} \times(\nabla \times \boldsymbol{A})+\nabla(\eta \nabla \cdot \boldsymbol{A}),
$$

where the horizontal velocities will be given by the photospheric driver, as discussed in the previous section.

A main concern in our model is the time evolution of the photospheric magnetic field. By lowering the magnetic resistivity (see Sect.4.2.1), we protect the photospheric magnetic field from diffusion. However, the random photospheric velocities will eventually destroy it. For that reason, we have to properly update and stabilize the photospheric magnetic field of the active region by some fraction of the initial one. We introduce the relaxation time $\tau_{b}$, which is set to 10 mins for all our numerical experiments. The idea is that to calculate the evolution of the photospheric magnetic field, or the vector potential in this case, from time $t_{0}$ to $t_{0}+\delta t$ we have to interpolate between the vector potential at $t_{0}$ and the initial one $\boldsymbol{A}^{\text {init }}$,

$$
\frac{\partial \boldsymbol{A}}{\partial t}=\ldots+\frac{1}{\tau_{b}}\left(\boldsymbol{A}^{\mathrm{init}}-\boldsymbol{A}\right) .
$$

By setting $\tau_{b}=10$ min we ensure that after 10 min the diffused surface magnetic field will be updated by some fraction of the original one and the overall evolution of the active region will be roughly stabilized. That matches quite nicely the stable nature of observed solar active regions. 


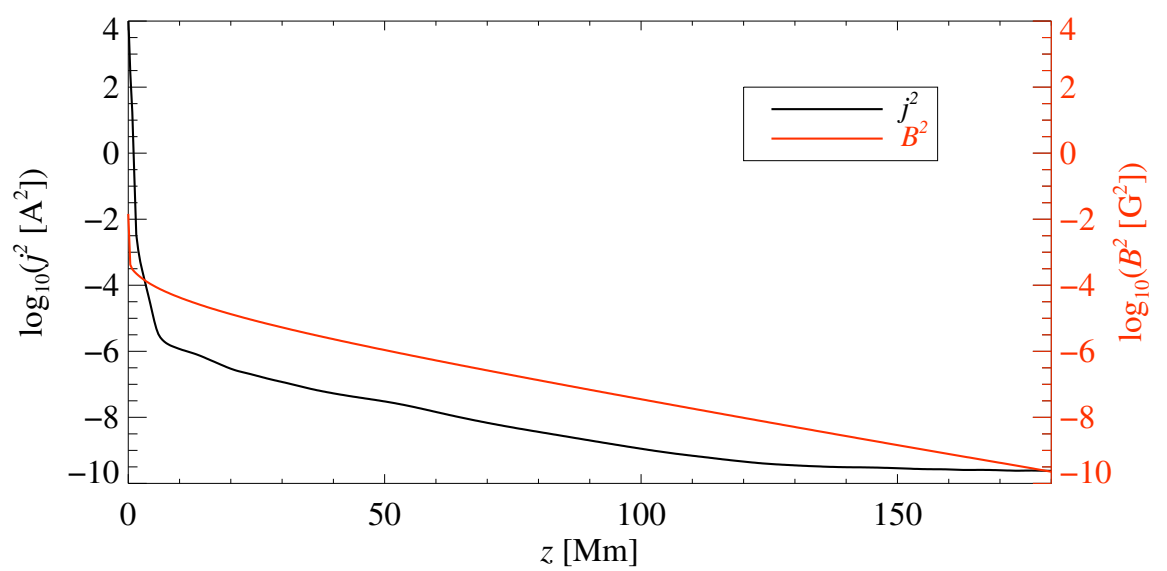

Figure 4.3: Horizontally averaged profile of current density squared $\boldsymbol{j}^{2}$ (black solid line) and horizontal average profile of magnetic field squared $\boldsymbol{B}^{2}$ (red solid line). Both are plotted for one snapshot in time for $t=200 \mathrm{~min}$.

\subsection{Verification of the numerical model}

To demonstrate the capabilities of our model, we show some basics results from a test run. For this test run, we use a magnetogram of a solar active region depicted in Fig. 4.2 as a bottom boundary condition. The computational box covers a surface area of 200x200 $\mathrm{Mm}^{2}$, and it also extends $200 \mathrm{Mm}$ in height. The magnetic field strength of the active region is $5 \mathrm{kG}$, and the overall surface magnetic flux is roughly $\Phi=6 \times 10^{22} \mathrm{Mx}$. The magnetic field strength and the amount of surface magnetic flux of this particular active region are a bit larger than the values found for typical solar active regions (i.e. 2-3 kG and $\Phi \simeq 10^{20}$ to $10^{21} \mathrm{Mx}$ ). Active regions of this size and strength could form in the Sun, maybe during its maximum activity, or in other stars more active than the Sun. The initial conditions, the choice of parameters, and the photospheric driver were discussed in the previous sections. In this section, we show horizontal averages of different quantities such as temperature, density, and Poynting flux. We also show signatures of nanoflares in the simulated corona and EUV synthesized emission plots of the coronal loops.

\subsubsection{Ohmic heating}

The coronal heating originates from the Ohmic dissipation of the induced currents. The large value of the plasma $\beta$ in the photosphere ensures that the magnetic field is bounded to the fluid motion. The horizontal motion will twist the initially potential field leading to the creation of currents. The Poynting flux generated will convert into heating which will supply the necessary energy to increase the coronal temperature. The Ohmic or Joule heating rate will be,

$$
H=\eta \mu_{0} j^{2} .
$$

At the base of the corona and above, the plasma $\beta$ is generally assumed to be much smaller than unity. In this case, the magnetic field is dominating, and the plasma is bounded by the magnetic field. In this part of the computational box, we can assume the plasma to be 
force-free. In the force-free approximation, the Lorentz force is stronger than any other force so that $\boldsymbol{j} \times \boldsymbol{B}=0$. This would mean that either the are no currents, $\boldsymbol{j}=0$, or the currents are parallel to the magnetic field, $\boldsymbol{j} \| \boldsymbol{B}$. However, in the case of no currents, the field is potential, and thus no heating would be possible. In the alternative case, where currents are parallel to the magnetic field,

$$
\boldsymbol{j}=\alpha \boldsymbol{B} .
$$

The parameter $\alpha$ should be constant along each magnetic fieldline for a force-free case but can change from one fieldline to another. However, in the case where the coronal plasma is not entirely force-free this parameter, $\alpha$ is expected to vary even along a specific fieldline. The result from Eq. (4.31) should be treated with caution. For an almost force-free state currents can lie almost parallel to the magnetic field. However, this is not the only option. As an alternative, it is proposed by Peter et al. (2015) the existence of "small currents" which can have an arbitrary angle with the magnetic field. For these "small currents", the Lorentz force does not vanish. However, as long as the magnetic field associated with the "small currents" is smaller than the potential field, then the force balance $\boldsymbol{j} \times \boldsymbol{B}=0$ is satisfied. The case of "small currents" is in most cases ignored but can have a significant effect on the calculated heating rates (see e.g. Peter et al. 2015).

To study if the coronal plasma is close to a force-free state, we plot in Fig. 4.3 the horizontally averaged profile of the current density squared $\boldsymbol{j}^{2}$, and the magnetic field squared $\boldsymbol{B}^{2}$ as a function of height $z$. Both $\boldsymbol{j}^{2}$ and $\boldsymbol{B}^{2}$ drop exponentially from the photosphere until the transition region. The strongest heating is located at the photosphere and low chromosphere. That is because of the high plasma $\beta$ plasma leading to a large stressing of the magnetic field. In the coronal part, the scale height is quite large, and thus, the exponential drop is not that strong. At the corona, $\boldsymbol{j}^{\mathbf{2}}$ and $\boldsymbol{B}^{\mathbf{2}}$ drop in the same fashion and even with the same scale height. Hence, $\boldsymbol{j}^{2}$ and $\boldsymbol{B}^{2}$ are found to be proportional. Our result suggests that the corona is indeed at a state close to the force-free. That was proposed by Schrijver et al. (1999) and later it was confirmed with the data provided by TRACE (Aschwanden et al. 2001). This result was also confirmed by other numerical and observational studies (Mandrini et al. 2000; Démoulin et al. 2003; Gudiksen and Nordlund 2005a).

For the force-free approximation to hold, it is assumed that the plasma $\beta$ in the corona is much smaller than unity. However, from observational and analytical calculations, it is suggested that plasma $\beta$ does not necessarily have to be small and can reach up to a few percent or even reach larger than unity (Peter et al. 2015). Given the uncertainties that enter the calculation of the plasma- $\beta$, the coronal plasma can be considered to be approximately force-free. However, the readers should keep in mind that the coronal plasma $\beta$ could have larger values, but it is left for future studies to investigate this problem even further.

\subsubsection{Coronal temperature and density}

A way to test the validity of our model is to show the temperature and density stratification of the simulated solar atmosphere. The energy flux is generated by the photospheric motions, and the radiative losses extract this energy out of the system. After the start of our simulation, we have to let it evolve until it reaches a quasi-stationary state. At that state, the simulation is independent of the initial conditions. This relaxed state is reached 

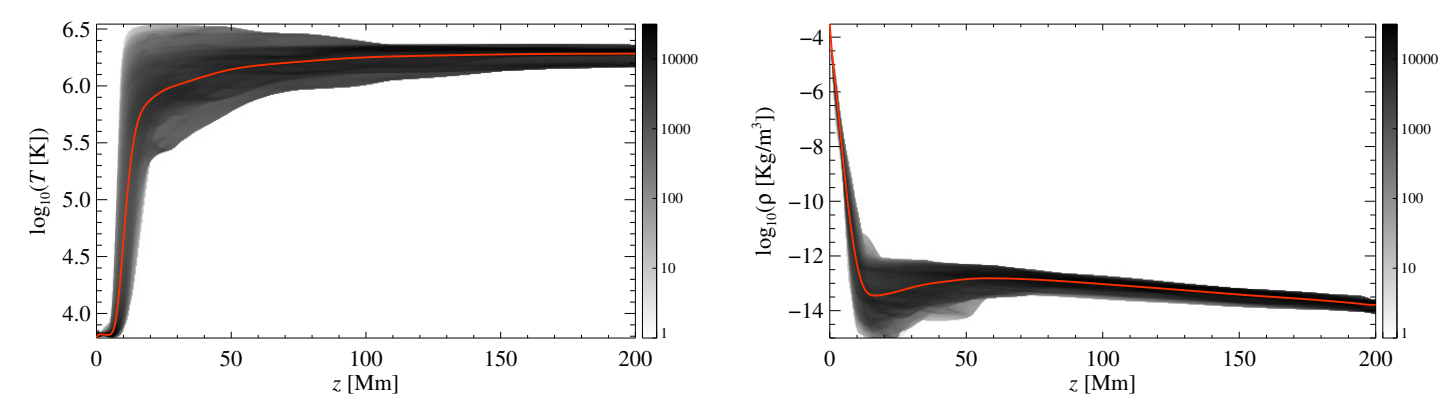

Figure 4.4: Probability density function and horizontally averaged temperature and density as a function of height for one snapshot at $t=200 \mathrm{~min}$. Left: Red solid line represents the horizontally averaged temperature. The grey area represents the probability density function for temperature. Right: Red solid line shows the horizontally averaged density profile. The grey area represents the probability density function for mass density.

after around 2 solar hours for this specific run. In Fig. 4.4 we plot the temperature and density stratification at one snapshot in time for $t=200 \mathrm{~min}$.

Our numerical model shows a stratification of the solar atmosphere similar to Vernazza et al. (1981). The various quantities such as temperature and density vary on small spatial scales and show a highly dynamic behavior. However, to extract useful information on the coronal behavior, we require the computation of the horizontal averages. The advantage of horizontal averages is the minimal dependence on time. The horizontal average temperature (see red solid line in Fig. 4.4) increases from a photospheric value of $6000 \mathrm{~K}$ to a coronal value of $2 \mathrm{MK}$. The base of the corona can be defined at the height $z \simeq 20 \mathrm{Mm}$. The black area depicted in Fig. 4.4 shows the probability distribution function of the temperature. It shows how temperature is spatially distributed at a specific height. We can see that there are parts of the box, especially in the transition region, where temperature can reach even higher values than $3 \mathrm{MK}$. Similar for the density, we see a drop of 8 orders of magnitude from the photosphere to the transition region. In the coronal part, the horizontal average density is on the order of $10^{-13} \mathrm{~kg} / \mathrm{m}^{3}$ which is very close to the observed solar coronal values.

These results have been confirmed many times by other similar studies (Gudiksen and Nordlund 2005b,a; Bingert 2009; Warnecke and Bingert 2020). Here we demonstrate the ability of our model to reproduce some of the characteristics of the solar corona. As such, it can be used to understand the stellar coronae, which we are going to discuss in the next chapters.

\subsubsection{Coronal energy flux}

The energy flux injected from the bottom boundary is converted into heat. This energy flux, known as Poynting flux, is responsible for the heating and the temperature increase of the coronal plasma. Due to the low-density environment of the solar corona, only a small fraction of the Poynting flux is enough to heat the plasma to temperatures on the order of a million Kelvin degrees. The Poynting flux is defined as,

$$
\boldsymbol{S}=\eta \boldsymbol{j} \times \boldsymbol{B}-\frac{1}{\mu_{0}}(\boldsymbol{u} \times \boldsymbol{B}) \times \boldsymbol{B} .
$$




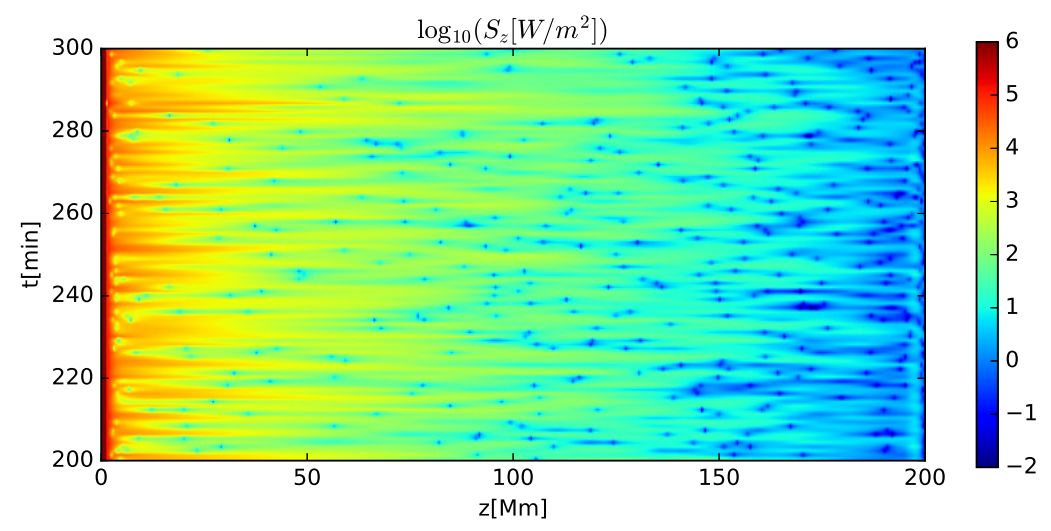

Figure 4.5: Horizontally averaged vertical Poynting flux as a function of height $\mathrm{z}$ and time. The time range is chosen during the relaxed state of the simulation.

In Fig. 4.5 it is illustrated how the horizontal average of the vertical Poynting flux $\left\langle S_{z}\right\rangle$ changes with height and time during the quasi-stationary state of the simulation. It is shown that the Poynting flux will not drop in a continuous way but rather in an intermittent. That would mean that the energy will be released in a storm of pulses, and the heating will also be intermittent (Bingert and Peter 2011). This storm of heating pulses could be associated with the nanoflare storm proposed by Parker (1972) (see also Sect. 4.6.4).

In Fig. 4.6 we depict as a black dashed line the horizontal average absolute vertical Poynting flux $\left\langle S_{z}\right\rangle$ for one snapshot in time for $t=200 \mathrm{~min}$. The Poynting flux Eq. (4.32) consists of two terms. The first term $\boldsymbol{j} \times \boldsymbol{B}$ is related to the currents. The currents will be strong, mainly at the lower parts of the atmosphere and near the bottom boundary. Because of the high plasma density at that specific part, the currents will not contribute significantly to the heating. For the upper atmosphere, the currents will drop exponentially, and thus $\boldsymbol{j} \times \boldsymbol{B}$ term is insignificant and can be neglected. The second term $(\boldsymbol{u} \times \boldsymbol{B}) \times \boldsymbol{B}$ is related to the plasma motion. Due to the presence of strong currents at the lower atmosphere, this term does not contribute to the Poynting flux. However, at the coronal part of the atmosphere, as the plasma beta also increases and currents get weaker, $(\boldsymbol{u} \times \boldsymbol{B}) \times \boldsymbol{B}$ term dominates and is upwards directed.

The Ohmic dissipation provides the energy needed to heat the coronal plasma. The energy flux $Q(z)$ gives the energy per unit time that crosses a unit plane at a specific height $z$. It is derived by integrating the Ohmic heating rate from a specific height $\mathrm{z}$ to infinity. The infinity in our simulation is considered to be the upper part of the numerical box. So the heating above height $z$ is because of the energy flux that crosses from that height. Mathematically, the flux $Q$ is defined as,

$$
Q(x, y, z)=\int_{z}^{\infty} \eta \mu_{0} \dot{j}^{2}\left(x, y, z^{\prime}\right) d z^{\prime} .
$$

The horizontal average of the energy flux density $\langle Q(z)\rangle$ is depicted in Fig. 4.6 as a red solid line. The average energy flux decreases from $10^{8} \mathrm{~W} / \mathrm{m}^{2}$ in the photosphere to roughly $100 \mathrm{~W} / \mathrm{m}^{2}$ at the base of the corona. This low value of the average energy flux proves to be enough to heat and sustain a MK hot corona for long timescales (see e.g. Withbroe and Noyes 1977). 


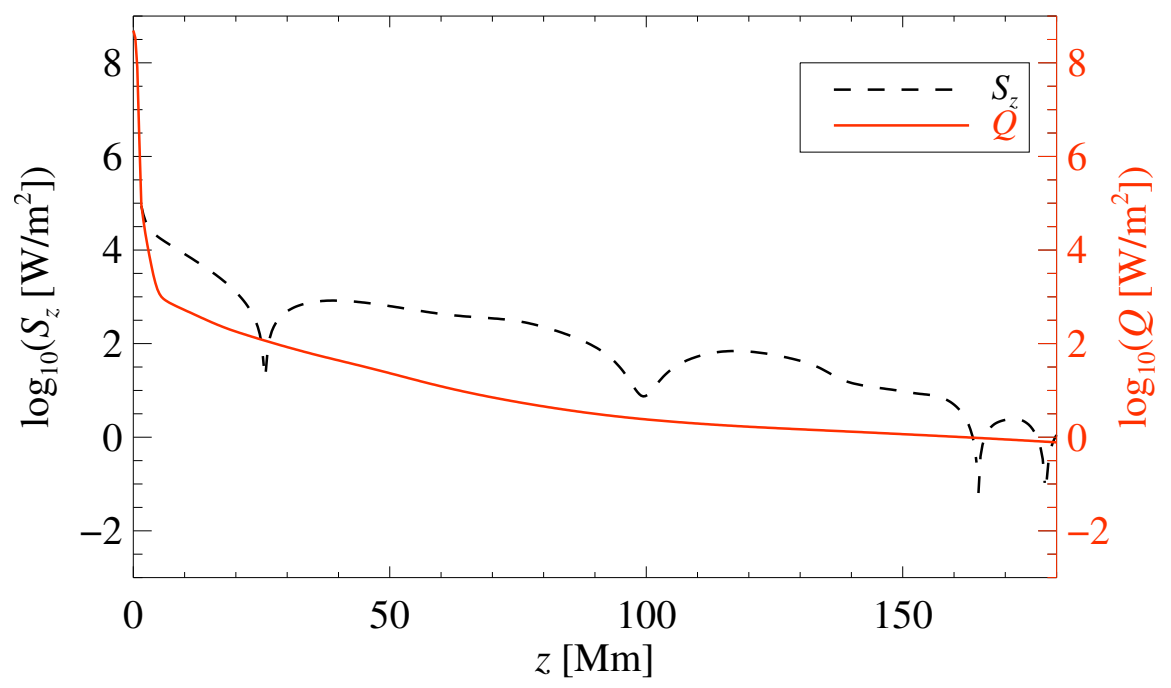

Figure 4.6: Horizontally averaged absolute vertical Poynting flux (back dashed line) and the energy flux density $Q(z)$ (red solid line). Both are plotted for one snapshot in time for $t=200 \mathrm{~min}$.

Finally, an equally interesting aspect is the correlation between the average vertical Poynting flux $\left\langle S_{z}\right\rangle$ and the average energy flux $\langle Q(z)\rangle$ as it is demonstrated in Fig. 4.6. The heating is provided by the conversion of the electromagnetic energy (i.e. the Poynting flux) via the Ohmic dissipation mechanism (Bingert and Peter 2011). Consequently, the averaged energy flux $\langle Q(z)\rangle$ is expected to decrease roughly in the same fashion as the Poynting flux.

\subsubsection{Nanoflares}

According to the nanoflare scenario, the energy in the corona is released in a storm of small flaring events. The time duration and the intensity of each event might vary. One single event does not have enough energy to heat the corona. However, a large number of events combined could provide the necessary energy for coronal heating. With the limited spatial resolution of the observational instruments, the existence of nanoflares has not been confirmed yet.

The spatial resolution in our numerical model is not large enough to resolve the nanoflares, but we can still measure their signature. In general, the horizontally averaged profiles of the heating rate or the Poynting flux will be roughly constant in time. However, this is not the case if we focus on a specific location, as it can be seen in Fig. 4.5. We expect the heating rate to show a very dynamic temporal evolution. To illustrate that, we plot the temporal evolution of the horizontal average heating rate in the corona at $z=39$ $\mathrm{Mm}$ (see Fig. 4.7). Even though the time variation is large, we can distinguish four events. The time duration of each of these four heating events varies from 6 minutes to 11 minutes. The time frame shown here is during the relaxed state of the simulation. The total energy release of each event is summarised in Table 4.1. We compute the total energy as,

$$
E=\iint \eta \mu_{0} \dot{j}^{2} d V d t \quad[J]
$$




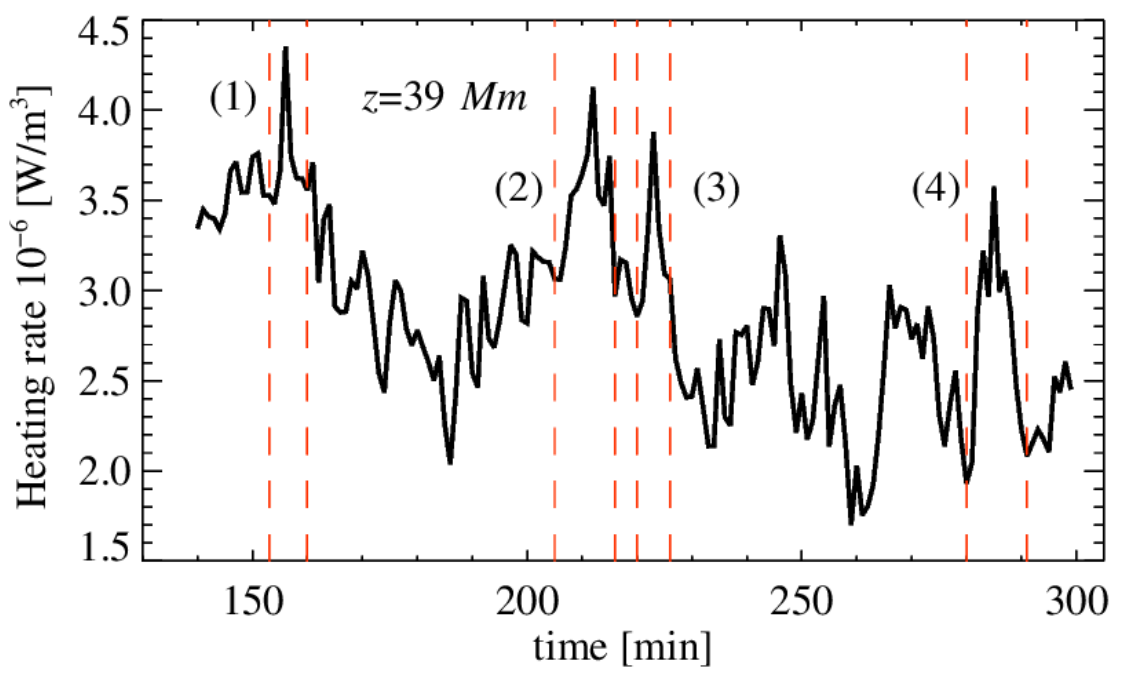

Figure 4.7: Horizontally averaged Ohmic heating rate evolution as a function of time for $z=39 \mathrm{Mm}$. The numbers (1)-(4) show the various events which are considered to be nanoflares. The red dashed lines indicate the time duration of each event.

The goal is to determine if any of this event can be characterized as a nanoflare.

The most promising events found in this specific simulation, which can be associated with nanoflares, are the events labeled as (1) to (4) depicted in Fig. 4.7. The energy release of these four events is in the range of $10^{19} \mathrm{~J}$ to $10^{20} \mathrm{~J}$ (see Table 4.1). A typical nanoflare on the Sun is expected to have energy on the order of $10^{17}$ to $10^{19} \mathrm{~J}$ (Aschwanden 2005). Events (1), (3), and (4) are inside this range and can be considered nanoflares. Event (2) has slightly larger energy expected by a typical nanoflare. The large duration of the event can explain the higher energy release. Nevertheless, we can consider three out of the four events to be associated with a nanoflare storm. The nanoflare storms might appear in different heights of the atmosphere, and it is expected to be the main mechanism of heating the chromosphere or the corona. The nanoflare storms in our numerical model appear self consistently, and it has been also confirmed by other studies (see e.g. Bingert 2009).

The nature of our numerical model does not allow for the existence of stronger flares. We use a stable active region that is twisted around by the granular motions. However, even these storms of weak nanoflare events provide enough energy to build a stable, hot corona. We can assume that a similar storm of flaring events, but with much higher intensity, will operate in other stars as well. Currently, the nanoflare model is considered to be the best candidate to explain the high coronal temperature.

Table 4.1: Duration and energy of the four events depicted in Fig. 4.7.

\begin{tabular}{ccc}
\hline \hline $\mathrm{N}$ & duration [s] & energy [J] \\
\hline Event 1 & 420 & $8.3 \cdot 10^{19}$ \\
Event 2 & 660 & $1.2 \cdot 10^{20}$ \\
Event 3 & 360 & $6.3 \cdot 10^{19}$ \\
Event 4 & 660 & $9.1 \cdot 10^{19}$ \\
\hline
\end{tabular}




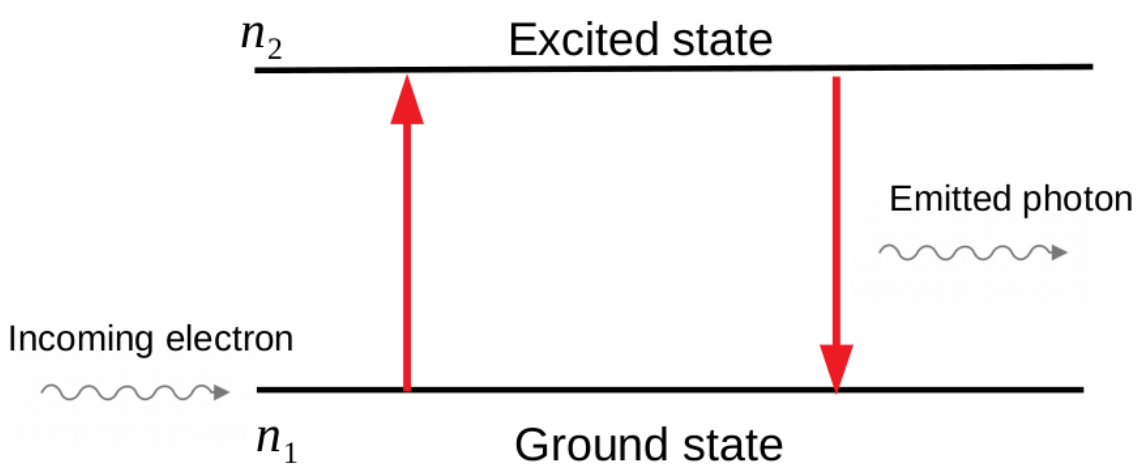

Figure 4.8: Sketch of the two level model. $n_{1}$ is the electron density at the ground state and $n_{2}$ is the electron density at the first excited state. The incoming electron will excite the ground state electron. The spontaneous emission will de-excite the electron and a photon will be emitted.

\subsubsection{Synthetic coronal emission}

To compare with observations of the solar corona, we need to calculate the emission at each grid point in our box. The line of sight synthetic emission from the simulations will match quite well the solar observations.

The modeled corona is considered to be optically thin. In this case, the most important processes in the radiation will be the bound-bound emission, free-bound emission, and Brehmstrahlung. We assume an ionization equilibrium to be able to calculate the emission at each grid point. We consider an electron number density $n_{1}$ at the ground state and an electron number density $n_{2}$ at the first excited state as it is depicted in Fig. 4.8. We primarily have excitation by an electron collision. In this case, the electron will jump from the ground state to the first excited state. A spontaneous emission will de-excite the electron back to the ground state, and a photon will be emitted (see Fig. 4.8). The emissivity at a specific wavelength in units of energy per time and volume will be,

$$
\epsilon_{i}=h v n_{2} A_{21} .
$$

Where $i$ refers to a specific wavelength, $h$ is the Planck constant, $v$ is the photon frequency, $n_{2}$ is the electron density in the excited state, and $A_{21}$ is the Einstein coefficient for spontaneous emission. The previous equation can be written as a chain of ratios,

$$
\epsilon_{i}=h v \frac{n_{2} A_{21}}{n_{e} n_{\mathrm{ion}}} \frac{n_{\text {ion }}}{n_{e}} \frac{n_{e}}{n_{\mathrm{H}}} \frac{n_{\mathrm{H}}}{n_{e}} n_{e}^{2},
$$

where $n_{\text {ion }}$ is the number of ionised atoms, $n_{\mathrm{H}}$ is the number density of hydrogen and $n_{e}$ is the electron number density at the corona. The number density of the excited state $n_{2}$ is proportional to the electron density since we have excitation by electron collision. The ionization ratio $n_{H} / n_{e}$ depends on the degree of ionization and can be determined from the CHIANTI database (Dere et al. 1997). The coronal plasma is considered to be fully ionized and the value of $n_{H} / n_{e}$ is roughly 0.8 . The ratio $n_{e} / n_{H}$, also known as abundance, is constant in the whole computational box tabulated also in the CHIANTI database. Finally, we end up with two temperature-dependent ratios. The first one is 
$C(T)=n_{2} A_{21} / n_{e} n_{\text {ion }}$ and the second one is $f(T)=n_{\text {ion }} / n_{e}$. From these two ratios, the $f(T)$ has a strong peak at a specific temperature. On the other hand, $C(T)$ is more spread out making, therefore, $f(T)$ the dominant term. The whole chain of ratios is also called the contribution function and has a weak dependence on density $n_{e}$ but a strong dependence on temperature $T$. Eq. (4.36) can be expressed in a more compact way,

$$
\epsilon_{i}=G_{i}(T) n_{e}^{2} .
$$

The intensity at a specific wavelength is,

$$
I_{i}=\int \epsilon_{i} d h=\int G_{i}(T) n_{e}^{2} d h,
$$

where $d h$ can be in any direction along the line of sight, i.e. $x, y, z$. To calculate the emissivity, we first need the spectra from a telescope and find the contribution function for each line using the CHIANTI database (Dere et al. 1997). If we sum up the emission for each line we get the temperature response function of a telescope $R(T)=\sum_{i} G_{i}(T) n_{e}^{2}$. To find the total emission for a specific wavelength range of a spectrum,

$$
I=\int R(T) n_{e}^{2} d h
$$

The electron's number density $n_{e}$ is calculated from the mass density $\rho$ we get as an output from the numerical model. Finally, the line of sight (los) emission at each direction will be,

$$
I_{x}=\int R(T) n_{e}^{2} d x, \quad I_{y}=\int R(T) n_{e}^{2} d y, \quad I_{z}=\int R(T) n_{e}^{2} d z .
$$

We can test our model by integrating the emission along a line of sight in all directions as it is described by Eq. (4.40). Since we consider an optically thin plasma, we can replace the integrals with a summation over all the grid points. For the temperature response function $R(T)$, we use the one from the AIA 171 channel. This channel represents the EUV emission of the corona at $171 \AA$ A. Fig. 4.9 illustrates the synthetic EUV emission as it would have been observed by AIA onboard SDO for three different snapshots in time ( $t=170 \mathrm{~min}, t=200 \mathrm{~min} t=230 \mathrm{~min}$ ). The EUV emission of AIA 171 originates from the Fe IX, which is found in the corona and has a peak temperature at $\log (T /[K])=5.9$. Here we show the emission in three different lines of sight directions. The left panel shows a view along the $x$-direction, the middle panel shows a top view (z-direction), and the right panel shows a side view ( $y$-direction). The left and right panels can be considered solar limb observations, whereas the middle panel corresponds to disk center observations. The top and side view (see Fig. 4.9 middle and right panel) show the formation of loop-like structures in the solar corona. The coronal loops appear to have different lengths and brightness. Specifically, in the top view, we see that those coronal loops are rooted at the two opposite magnetic polarities of the underlying active region. Towards the upper part of the box, the corona appears to be diffused. The white rectangular box in the three different snapshots in time shows the dynamic behavior of the coronal loops. We see that for $t=170 \mathrm{~min}$, there are no bright loops inside the white box, but they appear at $t=200$ min, and then they diffuse at $t=230 \mathrm{~min}$. The constant brightening and dimming of the coronal loops in our simulation is an indication of the dynamic nature of the solar corona. 

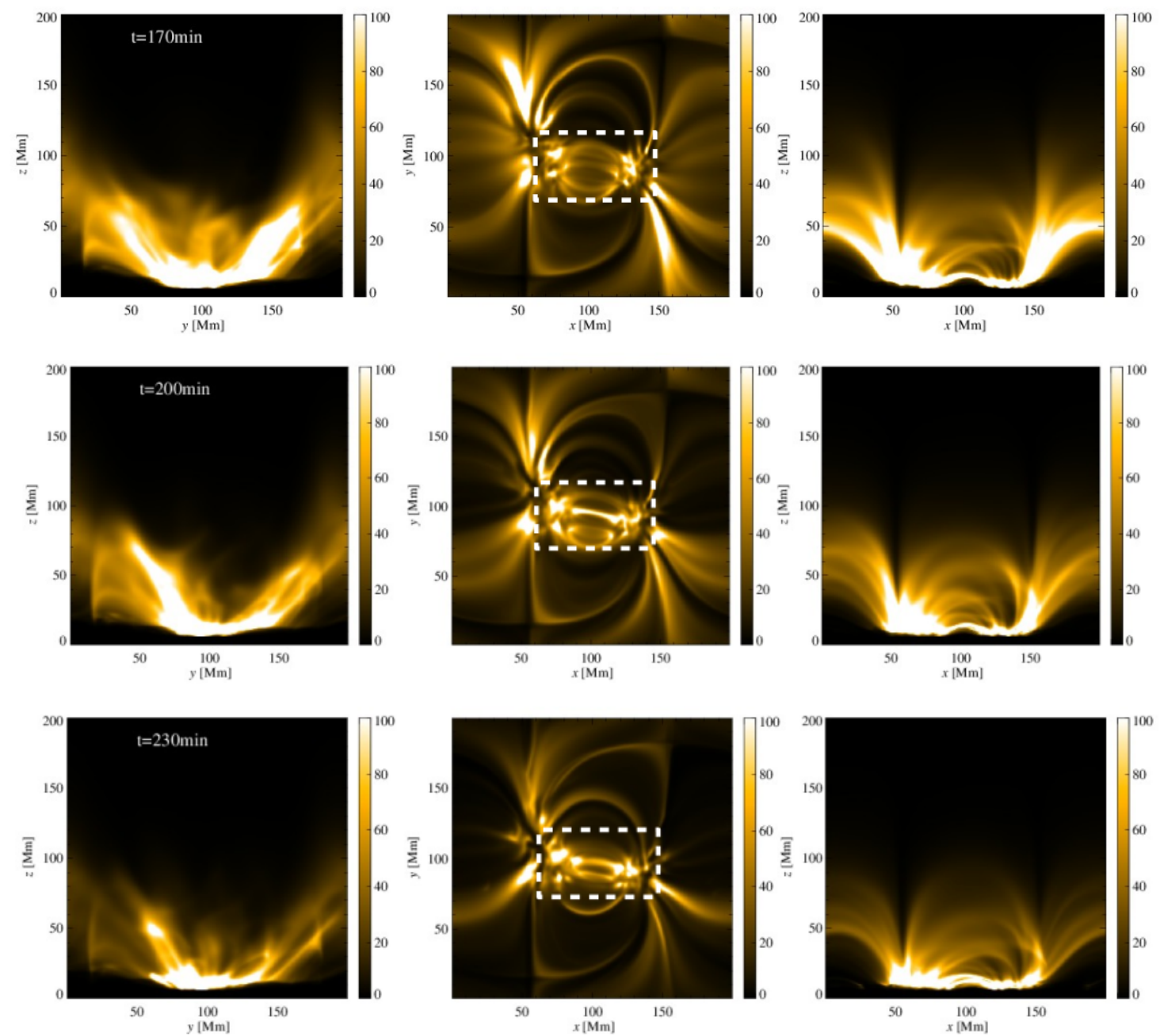

Figure 4.9: Synthetic EUV emission of the coronal plasma as it would have been observed in the AIA $171 \AA$ channel from AIA onboard SDO. The left column shows the emission in the $x$-direction. Middle column shows a top view of the emission at the $z$-direction. Right column shows a side view at $y$-direction. The white rectangular box shows the dynamic behavior of a bright coronal loop. 
This behavior is confirmed by various observations of solar coronal loops. It is speculated to be associated with the nanoflares, which probably heat the corona in numerous pulses of energy releases. To conclude, our numerical model successfully reproduces some of the aspects of the solar corona, and it can be used as a tool to extend the study in the coronae of stars more active than the Sun. 



\section{$5 \quad$ Stellar X-rays and magnetic activity in 3D MHD coronal models}

*Chapter 5 reproduces the paper Stellar X-rays and magnetic activity in 3D MHD coronal models by J. Zhuleku, J. Warnecke and H. Peter submitted in Astronomy \& Astrophysics. The abstract is not included here. I performed all the simulations, analysed and interpreted the data and wrote the first draft of the paper.

\subsection{Introduction}

Stellar coronal X-ray emission is observed to increase with stellar rotation rate (e.g. Pizzolato et al. 2003; Wright et al. 2011; Reiners et al. 2014; Magaudda et al. 2020). It is widely assumed that an increase in rotation could be responsible for stronger dynamo action leading to larger surface magnetic field. Some active stars (e.g. M dwarfs) that rotate rapidly (typical periods of 1 to 2 days) show high (average) photospheric magnetic field strengths which can reach up to $8 \mathrm{kG}$ or even more (Reiners 2012). Because of this increased photospheric magnetic field, we can expect that a stronger upward directed Poynting flux is generated that can heat the corona to higher temperatures and leads to stronger X-ray emission in the corona. An indication of such a behaviour has been found in stellar observations (e.g. Vidotto et al. 2014) revealing a close relation of the coronal $\mathrm{X}$-ray emission and the surface magnetic flux.

The scaling relationship between the coronal X-ray emission $L_{X}$ and the surface magnetic flux $\Phi$, have been extensively studied by employing solar and stellar observations. This relation follows a power-law, $L_{X} \propto \Phi^{m}$. In early studies, the power-law index $m$ was found to be close to unity (Fisher et al. 1998; Pevtsov et al. 2003), i.e. the X-ray radiation scales almost linear with magnetic flux. However, more recent studies suggest a much steeper power law with $m=1.8$ (Vidotto et al. 2014) or even steeper with $m=2.68$ (Kochukhov et al. 2020). The physical mechanism relating the observed X-ray emission to the surface magnetic flux is still under debate.

To study the impact of the surface magnetic field on the coronal X-ray emission in the environment of a realistic setup, the use of 3D magnetohydrodynamic (MHD) models is required. In addition, the $3 \mathrm{D}$ numerical simulations will provide a useful tool to further test the validity of a simplified analytical model. The main advantage of $3 \mathrm{D}$ models is the self-consistent treatment of the corona. The heating originates from the Ohmic dissipation of currents induced by photospheric magneto-convective motions. This drives the magnetic field similar to Parker's field line braiding (or nanoflare) model (Parker 1972, 1983). The Parker field-line braiding model has been extensively studied in numerical models. 
It was shown how the energy cascades in current sheets from large scales to dissipative scales before converting to heating (Rappazzo et al. 2008). In addition, 3D MHD simulations of footpoint motions have proved successful on forming a self-consistent corona (see e.g Gudiksen and Nordlund 2002, 2005a,b; Bingert and Peter 2011, 2013a; Hansteen et al. 2015; Dahlburg et al. 2016, 2018).

The numerical models are able to provide the necessary energy flux in the corona, which is sufficient to heat it to temperatures beyond $1 \mathrm{MK}$ and is consistent with observations (Bingert and Peter 2011; Hansteen et al. 2015). Furthermore, extreme ultraviolet (EUV) synthetic spectra from these 3D simulations can explain some aspect of the actual observations (Peter et al. 2004; Dahlburg et al. 2016; Warnecke and Peter 2019a). This confirms the validity and efficiency of Parker's field line braiding model to create a hot corona. These models can also be used to study the effects of magnetic helicity injection in the photosphere of active stars on the resulting coronal X-ray emission (Warnecke and Peter 2019b). This showed that an increase of photospheric magnetic helicity without changing the vertical magnetic field increases the coronal X-ray emission following simple power-law relations. However, the effect of the surface magnetic activity on the coronal X-ray emission in 3D MHD models of solar and stellar coronae has not been studied, yet.

In our study, we focus on the effect of the photospheric magnetic field strength on the coronal X-ray emission. This is motivated by the observation that stars more active than the Sun host stronger surface magnetic field. For that reason, we choose to increase the strength of the vertical surface magnetic field at the bottom boundary of our computational domain, i.e. we treat the peak (or average) magnetic field strength as a free parameter. All the other parameters remain the same in all numerical experiments. By varying only one parameter (i.e. the surface magnetic field) we can study the exact relation between magnetic flux and coronal emission. Other parameters, important for the coronal energy input, e.g. the photospheric velocity distribution, are observationally ill constraint for other stars and are thus not changed (or varied) in the present work. Our main objective is to relate the synthetic X-ray emission from the numerical models with the surface magnetic flux and relate this to the observed relationships. Furthermore, we will compare our numerical results to our earlier analytical model (Zhuleku et al. 2020) that is briefly summarized in Sect. 5.2.

\subsection{Analytical scaling relations}

The dependence of the coronal X-ray emission to surface magnetic field has been extensively studied for the Sun as well as for other stars (Fisher et al. 1998; Pevtsov et al. 2003; Vidotto et al. 2014; Kochukhov et al. 2020). In Zhuleku et al. (2020) we developed an analytical model to describe the $L_{\mathrm{X}} \propto \Phi^{m}$ relation, where $L_{\mathrm{X}}$ is the coronal X-ray emission and $\Phi$ the total surface unsigned magnetic flux.

Our model is based on the well known Rosner, Tucker \& Vaiana (RTV; Rosner et al. 1978) scaling laws. They derived scalings relating the volumetric heating rate and the loop length to the coronal temperature and pressure. Alternatively, we can express the RTV scaling laws in a way to relate temperature $T$ and density $n$ with the volumetric 
heating rate $H$ and loop length $L$,

$$
\begin{aligned}
T & \propto H^{2 / 7} L^{4 / 7}, \\
n & \propto H^{4 / 7} L^{1 / 7} .
\end{aligned}
$$

Using these scaling laws together with other relations, we derive an analytical expression for the X-ray emission $L_{X}$,

$$
\begin{aligned}
L_{X} & \propto \Phi^{m} \quad \text { with } \\
m & =\frac{\beta \gamma}{7}(2 \alpha+8)+\delta\left(\frac{4}{7}+\frac{1}{7} \alpha-\frac{8}{7} \beta \gamma-\frac{2}{7} \alpha \beta \gamma\right) .
\end{aligned}
$$

The power law index $m$ depends only on four parameters $\alpha, \beta, \gamma$ and $\delta$. The first, $\alpha$, is related to the temperature sensitivity of the instrument used for the X-ray observations. In general, the X-ray radiation is proportional to the density squared and to a temperaturedependent function $R(T)$. This function $R(T)$ is the sum of all the contribution functions of emission lines and continua, known also as temperature response function, and differs from one instrument to the next. We found that the temperature response function for temperatures below $\log _{10} T[K]=7$ can be expressed as a power-law (cf. Zhuleku et al. 2020, their Fig.1),

$$
R \propto T^{\alpha} .
$$

The parameters $\beta$ and $\gamma$ characterize the relation of the of energy flux, or the vertical Poynting flux $S_{z}$, injected in the corona to the vertical surface magnetic field $B$ and the volumetric heating rate $H$, again through power-laws,

$$
\begin{aligned}
S_{z} & \propto B^{\beta} \\
H & \propto S_{z}^{\gamma} .
\end{aligned}
$$

The case $\beta=1$ represents Alfvén wave heating and for $\beta=2$ nanoflare heating (see also Sect. 5.6.1). The parameter $\gamma$ was considered to be unity, $\gamma=1$. Finally, $\delta$ relates the surface area covered by a magnetic structure (e.g. a whole active region) to the total magnetic flux,

$$
A \propto \Phi^{\delta}
$$

Solar studies have suggested a value of $\delta=0.819$ (Fisher et al. 1998).

In our analytical study, we found the power-law index $m$ to be in the range from roughly one to almost two (Zhuleku et al. 2020). This result agrees quite well with most observations, but at least one more recent observation finds an even steeper power law connection of $L_{X} \propto \Phi^{m}$ with $m$ of 2.68 (Kochukhov et al. 2020).

In the numerical study presented in the following sections, we will assume that the area covered by the magnetic field remains the same, i.e. the change of the surface magnetic flux is solely due to the (average) vertical magnetic field strength. This is equivalent to choose $\delta=0$ in Eq. (5.7). In this case we get a much steeper power-law in the analytical model, with $m$ up to 4 (Zhuleku et al. 2020, Sect. 5.2, Eq. 15). The numerical study in this paper will give a more detailed comparison to the observations that provide a good match to the observations of Kochukhov et al. (2020). 


\subsection{Numerical model setup}

\subsubsection{Basic equations}

Our model is based on the work of Bingert and Peter (2011, 2013b). We use the PencIL CoDE (Brandenburg et al. 2020), where we numerically solve the MHD equations from the photosphere up to the corona. The size of the computational box is $128 \times 128 \times 128$ grid points in Cartesian coordinates $(x, y, z)$, representing a $50 \times 50 \times 50 \mathrm{Mm}^{3}$ volume with a grid-scale of $390 \mathrm{~km}$ in all directions. The comparably small grid size size allows us to run a large enough number of numerical experiments to study the relationship between the magnetic activity and coronal emission.

The MHD equations are the continuity, momentum, energy and induction equation connecting density $\rho$, velocity $\boldsymbol{u}$, and temperature $T$ with the magnetic field $\boldsymbol{B}$ and pressure $p$ :

$$
\begin{gathered}
\frac{\mathrm{D} \ln \rho}{\mathrm{D} t}+\boldsymbol{\nabla} \cdot \boldsymbol{u}=0, \\
\frac{\mathrm{D} \boldsymbol{u}}{\mathrm{D} t}=\frac{1}{\rho}[-\boldsymbol{\nabla} p+\rho \boldsymbol{g}+\boldsymbol{j} \times \boldsymbol{B}+2 \nu \boldsymbol{\nabla} \circ(\rho \underline{S})], \\
\frac{\mathrm{D} \ln T}{\mathrm{D} t}+(\gamma-1) \boldsymbol{\nabla} \cdot \boldsymbol{u}= \\
=\frac{1}{c_{V} \rho T}\left[\eta \mu_{0} \dot{\boldsymbol{j}}^{2}+2 \rho v \underline{S}^{2}+\boldsymbol{\nabla} \cdot \boldsymbol{q}+L_{\mathrm{rad}}\right] .
\end{gathered}
$$

Here the Lagrangian derivative $\mathrm{D} / \mathrm{D} t$ is defined as $\mathrm{D} / \mathrm{D} t=\partial / \partial t+\boldsymbol{u} \cdot \boldsymbol{\nabla}$. The current density is given by $\boldsymbol{j}=\boldsymbol{\nabla} \times \boldsymbol{B} / \mu_{0}$. The polytropic index of an ideal gas is given by $\gamma=5 / 3$. We use a constant gravitational acceleration, $\boldsymbol{g}=(0,0,-g)$, with $g=274 \mathrm{~m} / \mathrm{s}^{2}, c_{V}$ is the specific heat capacity at constant volume, and $\underline{S}=\left(u_{i, j}+u_{j, i}\right) / 2-\delta_{i j} \boldsymbol{\nabla} \cdot \boldsymbol{u} / 3$ is the rate of the strain tensor. The resistivity $\eta$ and viscosity $v$ are constants through the whole computational box with values $\eta=v=5 \times 10^{10} \mathrm{~m}^{2} \mathrm{~s}^{-1}$. At the bottom boundary we reduce $\eta$ to prevent a too large diffusion of the photospheric magnetic field.

The magnetic field is calculated through the vector potential as,

$$
\boldsymbol{B}=\boldsymbol{\nabla} \times(\boldsymbol{A}+\boldsymbol{\nabla} \phi),
$$

which ensure that the $\nabla \cdot \boldsymbol{B}=0$ is satisfied at all times. For our simulations we choose the resistive gauge $\phi=\eta \boldsymbol{\nabla} \cdot \boldsymbol{A}$ so that the induction equation reads,

$$
\frac{\partial \boldsymbol{A}}{\partial t}=\boldsymbol{u} \times(\boldsymbol{\nabla} \times \boldsymbol{A})+\boldsymbol{\nabla}(\eta \boldsymbol{\nabla} \cdot \boldsymbol{A}) .
$$

To close the system of equations we need the equation of state for an ideal gas which connects the gas pressure with temperature,

$$
p=\frac{k_{B}}{\mu m_{p}} \rho T .
$$

Where $\rho$ is the density, $k_{B}$ is the Boltzmann constant, $\mu$ is the mean atomic weight and $m_{p}$ is the proton mass. 
The radiative losses $L_{\mathrm{rad}}$, are modeled with an optically thin approximation using the radiative loss function $P(T)$. The function $P(T)$ depends on the abundances as well as on the radiation processes that take place like the ionization rate and recombination rate (Meyer 1985; Murphy 1985; Cook et al. 1989). For a detailed discussion in the implementation of the radiative loss function in the code see Bingert (2009).

The (Spitzer) heat conduction along the magnetic field lines $\boldsymbol{q}$ reads,

$$
\boldsymbol{q}=K_{0} T^{5 / 2} \hat{\boldsymbol{b}}(\hat{\boldsymbol{b}} \cdot \nabla T)
$$

where $K_{0}=10^{-11} \mathrm{~W}(\mathrm{mK})^{-1}$ (Spitzer 1962) and $\hat{\boldsymbol{b}}$ is the unit vector of the magnetic field. To speed up the simulations, we replace Eq. 5.14 by a non-Fourier heat flux scheme. See Warnecke and Bingert (2020) for a detailed description and discussion. Additionally, we use a semi-relativistic correction to the Lorentz force similar to the work of Boris (1970), Gombosi et al. (2002) and Rempel (2017). For details of the implementation, see Chatterjee (2018) and Warnecke and Bingert (2020).

In order to avoid instabilities because of steep gradients in temperature and density, we include in Eq. (5.10) a (numerical) isotropic heat conduction term and a mass diffusion term (see Bingert 2009). We also include a shock viscosity term for numerical reasons.

\subsubsection{Initial and boundary conditions}

The simulations are driven by (horizontal) motions on the solar surface that drive the magnetic field anchored in the photosphere. For the spatial distribution of the vertical magnetic field we employ an observed solar magnetogram (see Fig. 5.1). This is a snapshot of the active region AR 11102 as observed with the Helioseismic and Magnetic Imager (HMI; Schou et al. 2012) on August 30th, 2010. A detailed description of a similar active region and how it is implemented in the model is discussed in Warnecke and Bingert (2020). These kind of magnetograms represent a typical situation for a solar active region and similar magnetograms have been used as input for data-driven simulations by earlier studies (see e.g. Gudiksen and Nordlund 2005b,a; Bingert and Peter 2011).

For the initial condition, we use a potential field extrapolation to fill the box with magnetic field. The initial temperature stratification follows a vertical profile mimicking the temperature increase into the solar corona. The initial density is calculated from hydrostatic equilibrium and the system is initially at rest with all velocity components set to zero.

In the horizontal $x-y$ plane, all variables are periodic. At the bottom boundary, the temperature $T$ and density $\rho$ have fixed values whereas the horizontal velocities, $u_{x}, u_{y}$ have zero vertical gradients. The vertical velocity $u_{z}$ is set to satisfy the divergence-free condition $\boldsymbol{\nabla} \cdot \boldsymbol{u}=0$. We also prescribe a photospheric driver which generates random photospheric velocity motions similar to the solar granular motion. As a result, we shuffle the footpoints of the magnetic loops mimicking the solar photospheric flows similar as in, e.g., Gudiksen and Nordlund (2002, 2005b,a). At the top boundary, all velocities components are zero. To prevent any heat flux going in or out of the computational domain, the gradients of temperature and density are set to zero. The magnetic field is potential both for the bottom and the top boundary. 


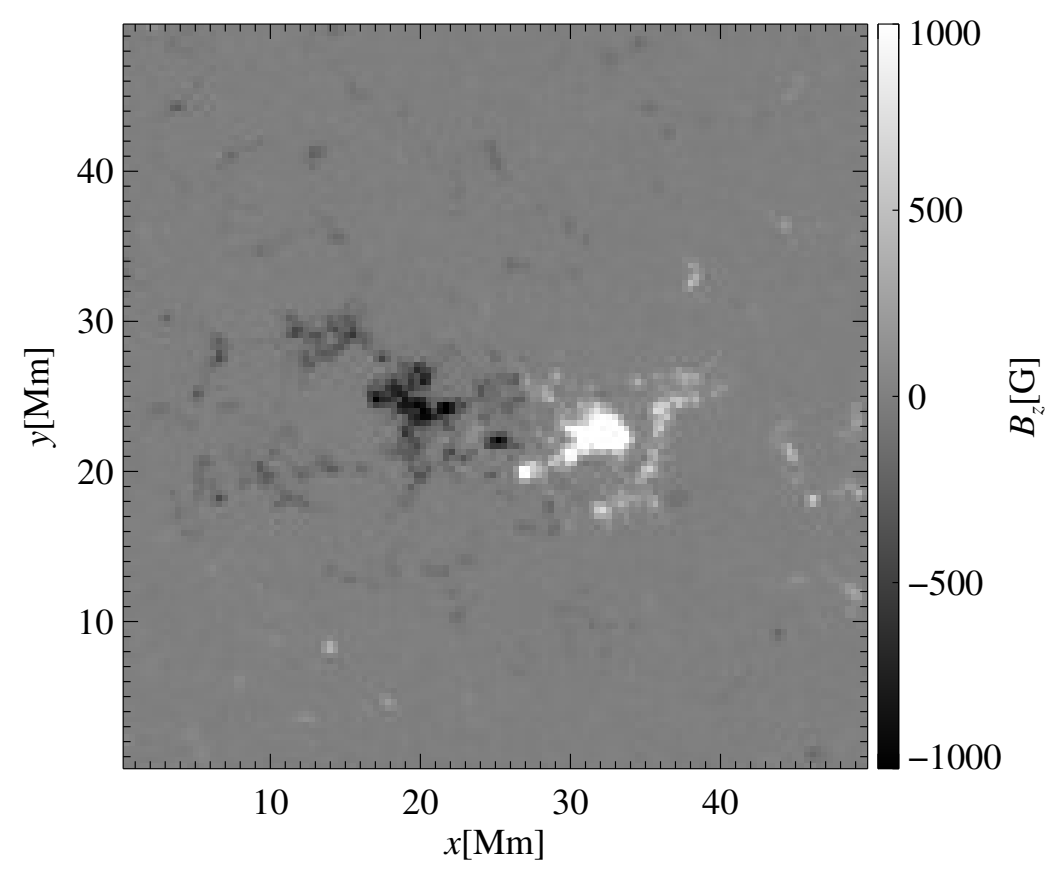

Figure 5.1: Initial vertical magnetic field at the bottom boundary of the simulation. This is based on a magnetogram from active region AR 11102 observed with HMI. See Sect. 5.3.2.

Table 5.1: Summary of numerical experiments. The photospheric magnetic field strength is increased by the factor a. $\Phi$ is the total surface unsigned magnetic flux.

\begin{tabular}{ccc}
\hline \hline Run & a & $\Phi[\mathrm{Mx}]$ \\
\hline 1B & 1 & $7.4 \times 10^{20}$ \\
2B & 2 & $1.5 \times 10^{21}$ \\
5B & 5 & $3.7 \times 10^{21}$ \\
10B & 10 & $7.4 \times 10^{21}$ \\
20B & 20 & $1.5 \times 10^{22}$ \\
\hline
\end{tabular}

\subsection{Numerical experiments}

\subsubsection{Setup}

The main idea of this work is to start with an active region hosting only a small amount of total (unsigned) magnetic flux and then run models with increasing magnetic flux. Our aim is to study how the increase of the photospheric magnetic field strength contributes to the heating, and thus the X-ray emission of the solar or stellar corona.

We report here on a set of five numerical experiments. The original total unsigned surface magnetic flux of AR11102 is around $7 \times 10^{20} \mathrm{Mx}$, which is a typical value of a small solar active region. We increase the flux by multiplying the $B_{z}$ component of the surface magnetic field by a constant ranging from one to twenty (see Table 5.1). The spatial structure of the magnetic field remains unchanged. In all cases, the total magnetic 
flux at the bottom boundary is zero, i.e. the surface magnetogram is balanced.

The heating of the coronal loops originates from the dissipation of Poynting flux into heat. More specifically, the conversion of the photospheric magnetic energy to thermal energy is due to the dissipation of the currents created by the random photospheric motions. The stronger the magnetic field, the more Poynting flux reaches the corona, resulting in a higher temperature and X-ray emission.

In our setup, the different total unsigned magnetic fluxes correspond to peak values of the magnetic field ranging from 1 to $20 \mathrm{kG}$ inside the spots. Hence our naming convention in Table 5.1. The value of $20 \mathrm{kG}$ is very high for a solar active region. Recent observations of solar active regions have measured maximum values of the magnetic field strength on the order of $8 \mathrm{kG}$ (Castellanos Durán et al. 2020). However, this high value was observed only at a very small area on the active region light bridge. Typically the peak magnetic field strengths on solar active regions are on the order of $2 \mathrm{kG}$ to $3 \mathrm{kG}$. Therefore, of the numerical experiments listed in Table 5.1, the 5B run can be considered to represent a typical solar active region, with a typical value for the total unsigned flux. Higher values of surface magnetic field could be a common feature, however, of very active stars (Reiners et al. 2014), so the runs 10B and 20B could be considered to be representative of more active stars.

The increase of the surface magnetic field is not the only way to increase the surface magnetic flux. Alternatively we could increase the horizontal extent of an active region while keeping the peak magnetic field strength the same. This will be the main focus of a future study.

\subsubsection{Synthesized emission: X-rays and EUV}

Coronal loops are mostly observed in extreme ultraviolet (EUV) wavelengths and X-rays because these wavelengths give access to the million $\mathrm{K}$ hot plasma in the corona, and in these wavelengths, the dilute corona is also visible in front of the solar (or stellar) disk that is very bright in the visible range. Therefore, we synthesize the emission in one EUV and one X-ray band. For the EUV band we choose the widely used $171 \AA$ band as seen by the Atmospheric Imaging Assembly (AIA; Lemen et al. 2012) onboard the Solar Dynamic Observatory (SDO; Pesnell et al. 2012). For the X-ray emission, we use the Al-poly filter of the solar X-ray telescope (XRT; Golub et al. 2007b) onboard the Hinode observatory(Kosugi et al. 2007).

As mentioned in Sect. 5.2, the optically thin radiative losses through lines and continua in the corona observed in a given wavelength band are given through

$$
\varepsilon=n_{\mathrm{e}}^{2} R(T)
$$

where $n_{\mathrm{e}}$ is the electron density and $R(T)$ the temperature response (or contribution) function. The response needs to be calculated for each instrument (or filter) by using the effective area depending on wavelength and the spectral lines forming in the wavelength region covered by the instruments. To calculate $R(T)$ for the $171 \AA$ channel of AIA and the Al-poly filter of XRT, we use the routines in the Chianti data base v9 (Dere et al. 1997, 2019 ) as they are available in the SolarSoft package ${ }^{1}$. The response functions $R(T)$ for

\footnotetext{
${ }^{1}$ http://www. lmsal. com/solarsoft
} 

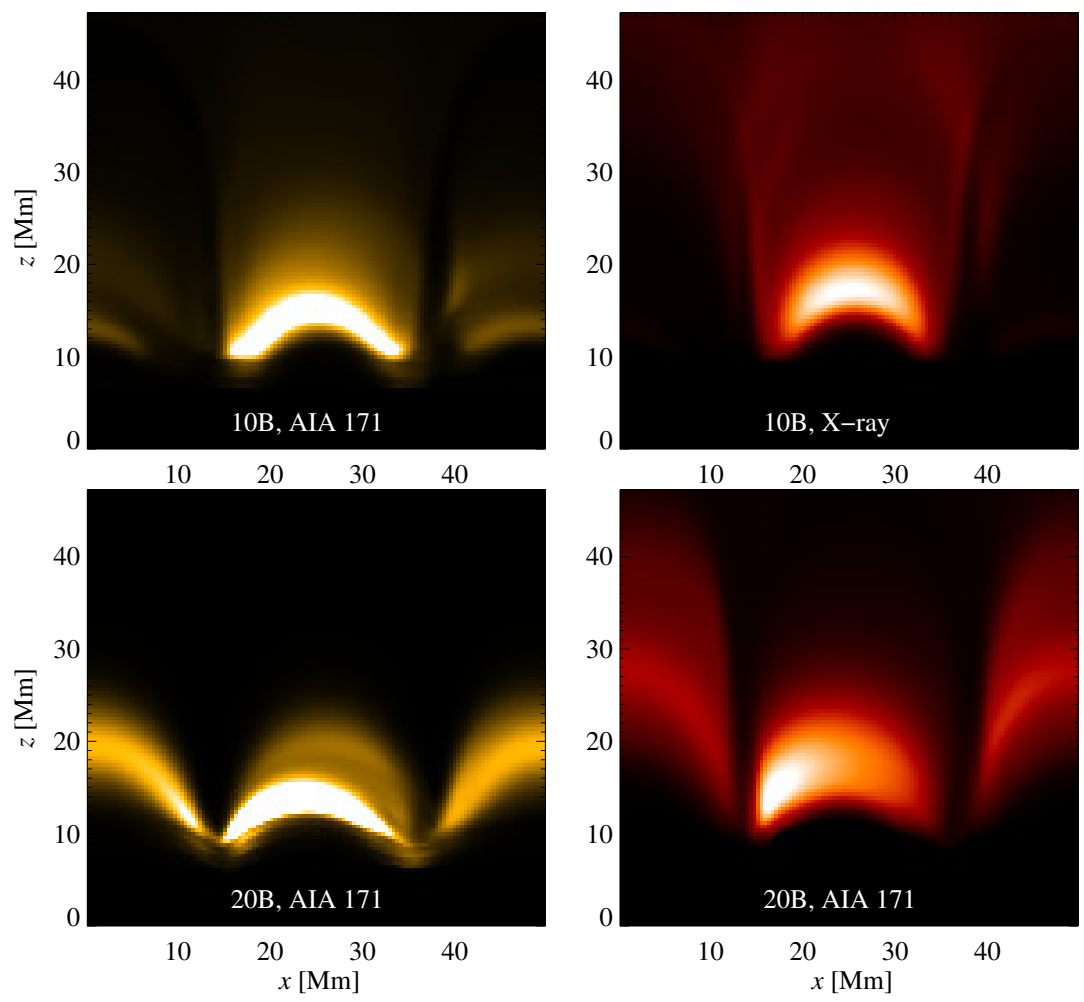

Figure 5.2: Side view of the computational domain showing coronal emission. The left two panels show the emission as it would be seen by the $171 \AA$ channel of AIA origination from around $1 \mathrm{MK}$. The right to panels show X-ray emission as seen by XRT sampling higher temperatures. Here we show snapshots of the two more active models, runs 10B and 20B. The emission here is integrated along the $y$ direction which corresponds to an observation near the limb (of the Sun or a star). The snapshot is taken at time at $t=230$ min, i.e. in the relaxed state. See Sect. 5.4.2.

AIA $171 \AA$ and XRT Al-poly are shown in Fig. 5.3. To calculate the synthetic images as they would be observed by AIA or XRT, $\varepsilon$ from Eq. (5.15) has to be integrated through the computational domain along the chosen line-of-sight. The samples for both channels for the 10B and 20B runs are shown in Fig. 5.2. These are integrated along the $y$ direction which would correspond to an observation near the limb.

\subsubsection{Horizontal averages}

The increase of the surface magnetic field results in an increase in temperature and density in the coronal part of the domain. We calculate the horizontal averages of temperature $T$, density $\rho$, and the vertical component of the Poynting flux $S_{z}$ and then average these in time for and interval of 1 hour. During this time interval the computation reached a relaxed state, i.e. the respective (spatially averaged) quantities show only rather small changes around a mean value (see Sect. 5.4.4 and Fig. 5.5). The average vertical stratification of $T, \rho$ and $S_{z}$ is shown in Fig. 5.4. 


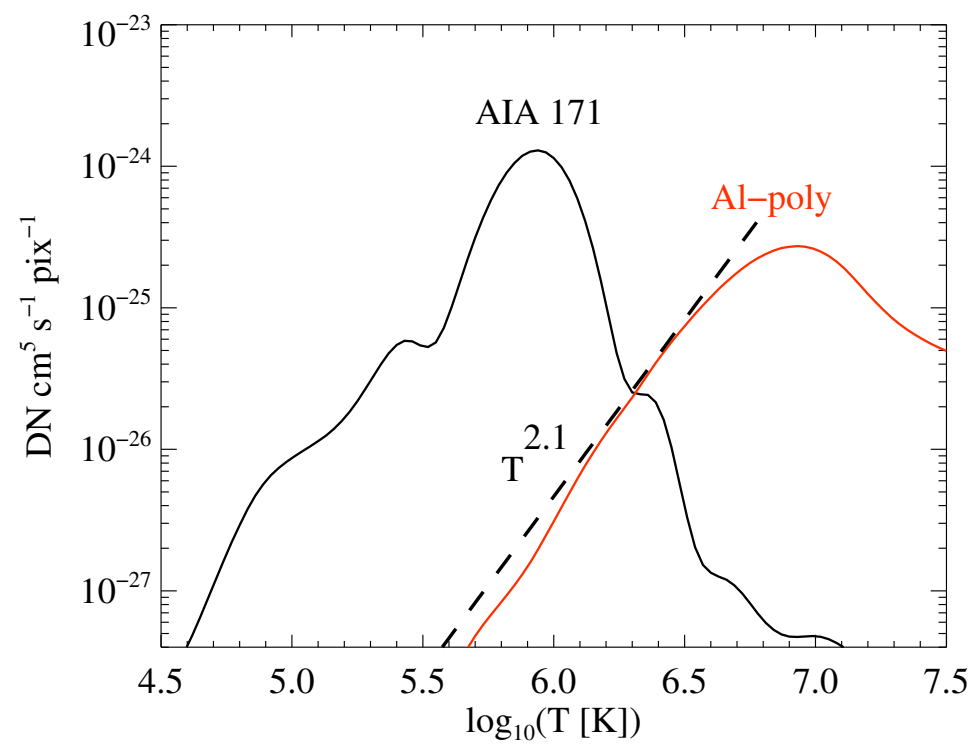

Figure 5.3: Temperature response function for the AIA instrument onboard SDO and the XRT onboard Hinode. The Black line shows the $171 \AA$ channel of the AIA, the red line shows the Al-poly filter of the XRT. Just for illustration, the dashed line indicates a power-law approximation to XRT at temperatures below $10^{7} \mathrm{~K}$. See Sect. 5.4.2.

\subsubsection{Average Poynting flux deposited in the corona}

The photospheric horizontal motions lead to an upward-directed flux of magnetic energy, the Poynting flux. Here we concentrate on its vertical component,

$$
S_{z}=\left.\eta(\boldsymbol{j} \times \boldsymbol{B})\right|_{z}-\left.\frac{1}{\mu_{0}}(\boldsymbol{u} \times \boldsymbol{B} \times \boldsymbol{B})\right|_{z}
$$

that is shown in Fig. 5.4c for the different runs. In the main part of the computational domain, the $u \times B \times B$ term dominates and (on average) is positive, i.e. upwards directed. The first term including the current, $j$, is significant only near the bottom where boundary effects of the driving cause high currents. Energetically, this is not relevant, because there the density is high enough that the heating through the currents has virtually no effect.

As we increase the total unsigned magnetic flux from one experiment to the next, the energy stored in the corona increases. The magnetic energy in excess of that of a potential field will be (partly) dissipated and converted into heat. The higher amount of dissipated (free) magnetic energy in the runs with higher magnetic flux leads to higher coronal temperatures and density (see Fig. 5.4a and Fig. 5.4b). This is just as expected from the RTV scaling laws (Eqs. 5.1 and 5.2) as we will discuss later in Sect. 5.6.2 (see also Fig. 5.8).

The Poynting flux of the 1B run at the base of the corona (i.e. at an average temperature of ca. $0.1 \mathrm{MK}$ ) barely reaches $50 \mathrm{~W} / \mathrm{m}^{2}$. Typical estimations based on observations suggest an energy requirement of around $100 \mathrm{~W} / \mathrm{m}^{2}$ for the quite Sun and $10^{4} \mathrm{~W} / \mathrm{m}^{2}$ above active regions (Withbroe and Noyes 1977). Therefore, we cannot expect this run with the lowest magnetic activity to produce a MK corona. 

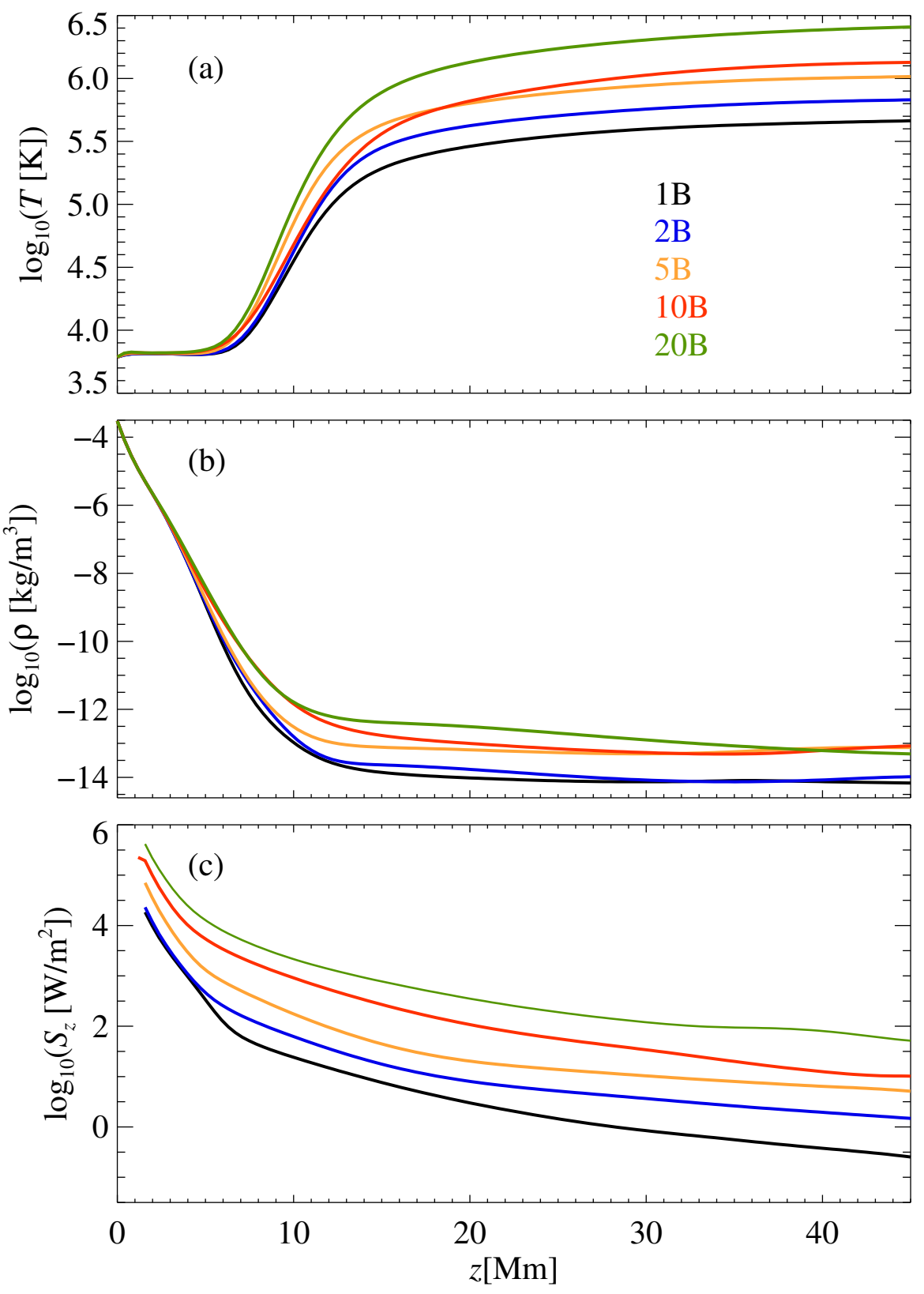

Figure 5.4: Horizontal averaged quantities as a function of height. We show temperature $T$ (panel a), density $\rho$ (panel b), and the vertical component of the Poynting flux $\mathrm{S}_{z}$ (panel c). The colors represent the different runs as indicated in the legend (cf. Table 5.1). The quantities are averaged horizontally for each snapshot and then in time for 1 hour (from times $3.5 \mathrm{hr}$ to 4.5 as indicated in Fig. 5.5 by the vertical dashed lines. For the Poynting flux we omitted the first 3 grid points that show boundary effects. See Sect. 5.4.3.

\subsubsection{Average temperature and density}

All of our simulations self-consistently form a hot upper atmosphere, where the temperature is about two orders of magnitude higher than at the surface (cf. Fig. 5.4a). A higher 
total unsigned flux in the photosphere (cased 1B through 20B) corresponds not only to higher Poynting fluxes, but also to higher temperatures and density. The values shown in Fig. 5.4a and $\mathrm{b}$ are averages only, so the peak values are significantly higher, up to $5 \mathrm{MK}$ and more.

The experiment with the least magnetic activity (run 1B) fails to create a millionKelvin hot corona, as expected. Still, we consider it in the analysis of the power-law relation in Sect. 5.5. The main focus of this work is to relate the coronal emission to the surface magnetic activity through a number of numerical experiments, and in this sense also a model that is not active enough to produce a MK corona gives valuable insight.

Besides the increased temperature and density, the models with higher magnetic activity also have the transition region located at lower heights. From this, it is clear that the height where the average temperature reaches $10^{5} \mathrm{~K}$ is lower for the runs with more magnetic flux. The higher energy input leads to a higher heat flux back to the Sun. Because the radiation is most efficient at lower temperatures (at $0.1 \mathrm{MK}$ and below), in equilibrium the transition region will be found at lower temperatures and thus higher densities where it can radiate the energy. The consequence is that the density (and the pressure) throughout the corona will be higher, just as seen in our simulations.

The average density profile $\rho$ displays similar (qualitative) behavior as the temperature. In the coronal part the density is high for the runs with higher magnetic flux (Fig. 5.4b). Following a steep drop over many orders of magnitude in the low atmosphere, the density remains almost constant in the coronal part. This is simply because of the large barometric (pressure) scale height at high temperatures. At $1 \mathrm{MK}$ this scale height is about $50 \mathrm{Mm}$ and thus comparable to the vertical extent of our computational domain, hence the horizontally averaged pressure and density are roughly constant in the coronal part of our box.

\subsubsection{Temporal evolution}

The quantities we consider in our model vary significantly with time, especially during the early phase of the simulations. To investigate the average behavior of our model we have to consider a time frame of the numerical model where the system reached a relaxed (or evolved) state. During that state, the quantities show (comparably small) variations around an average value.

To illustrate this, we first consider the total heating in the coronal part of the computational domain. We define the coronal part as the volume above the height where the horizontally averaged temperature is $10^{5} \mathrm{~K}$. Because the temperature gradient in the transition region around $10^{5} \mathrm{~K}$ is rather steep, the exact choice of this temperature does not matter. So we define the total coronal heating $H_{\text {tot }}$ as the volume integral over this coronal part, here symbolized by the subscript cor,

$$
H_{\mathrm{tot}}=\int_{\text {cor }} \eta \mu_{0} \dot{j}^{2} d V
$$

The temporal variation of the heating is shown in Fig. 5.5 for the five models with different magnetic activity. We see a clear ordering of the heating with the surface magnetic flux (increasing for run 1B through 20B), which we will discuss in more detail later in Fig. 5.7 and Sect. 5.6.1. In terms of the temporal evolution, we find that the heat input 


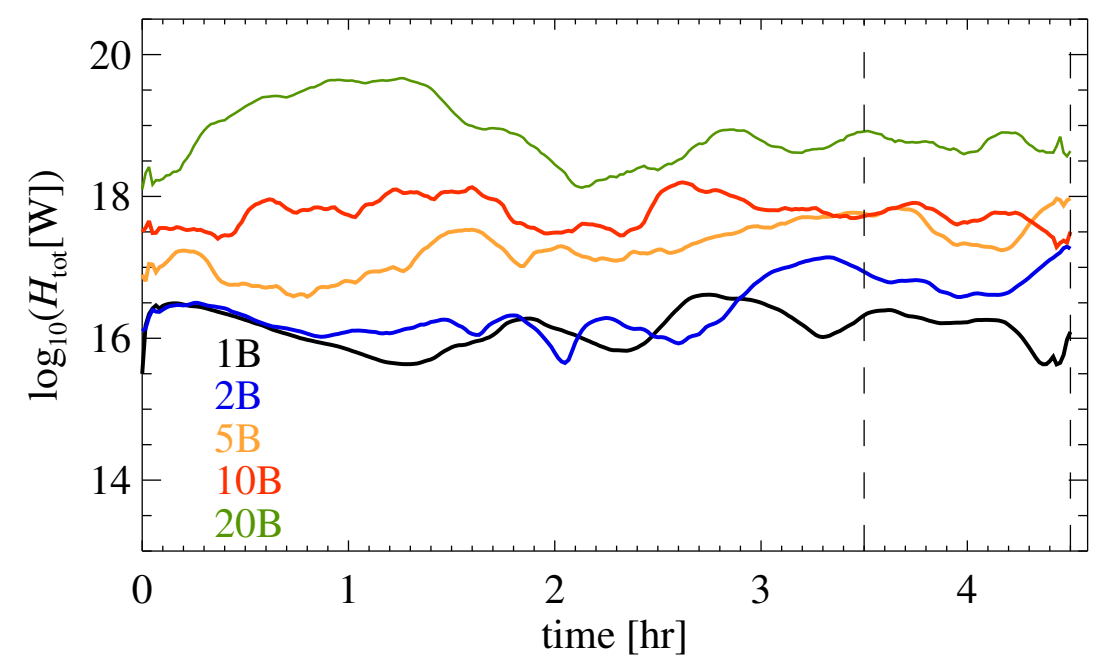

Figure 5.5: Total coronal heating $H_{\text {tot }}$ as a function of time. The vertical dashed lines indicate the time span used for the temporal averages. The colors represent the different runs as indicated in the legend (cf. Table 5.1). See Sec. 5.4.4.

reaches a relaxed state rather quickly, probably within less than an hour. This is expected, because the stresses applied on the magnetic field in the photopshere will propagate with the Alfvén speed. The corresponding Alfvén crossing time for perturbations to cross the whole box is on the order of minutes.

The situation for the relaxation time is different when considering the coronal emission in X-rays and EUV. Here it turns out that we have to wait for about 3 hours before the models reach a relaxed state. Mainly, this is because of the radiative cooling time under typical coronal conditions which is on the order of 1 hour (Aschwanden 2005). Therefore we will examine the temporal evolution of the coronal radiation in some more detail.

We now turn to the variability of the X-ray and EUV emission (see Fig. 5.6). Because the lower cool part of the atmosphere does not produce any significant amount of X-rays or EUV, we simply integrate the coronal emission over the whole computational domain. This is equivalent to the luminosity originating from the domain, $L_{\mathrm{X}}$ and $L_{\mathrm{EUV}}$. Because we use the temperature response functions for XRT and AIA (Sect. 5.4.2, Fig. 5.3), we get the counts per second as expected for the respective instrument from the whole loop in the box (cf. Fig. 5.2). For the comparison between the different model runs it is important that we use the same scale for the different models. Based on this, we see a clear scaling of the coronal emission with magnetic activity, in a similar way as we see it for the heat input. We will discuss this in more detail in Fig. 5.9 and Sect. 5.6.3.

Mostly, the coronal emission shows an initial drop on the timescale of almost an hour, in particular for EUV channel of AIA $171 \AA$ in the runs with low magnetic activity. This is because the atmosphere of the initial condition is rather hot, and the plasma is cooling down until it reaches a new equilibrium after a few coronal radiative cooling times (Fig. 5.6). Here we see that all model runs reach a relaxed state after about 2.5 to 3 hours. With some safety margin we can thus assume that from 3.5 to 4.5 hours the models have reached a relaxed state (see vertical dashed lines in Figs. 5.5 and 5.6). All the time averages discussed in our study are taken over this time frame. 

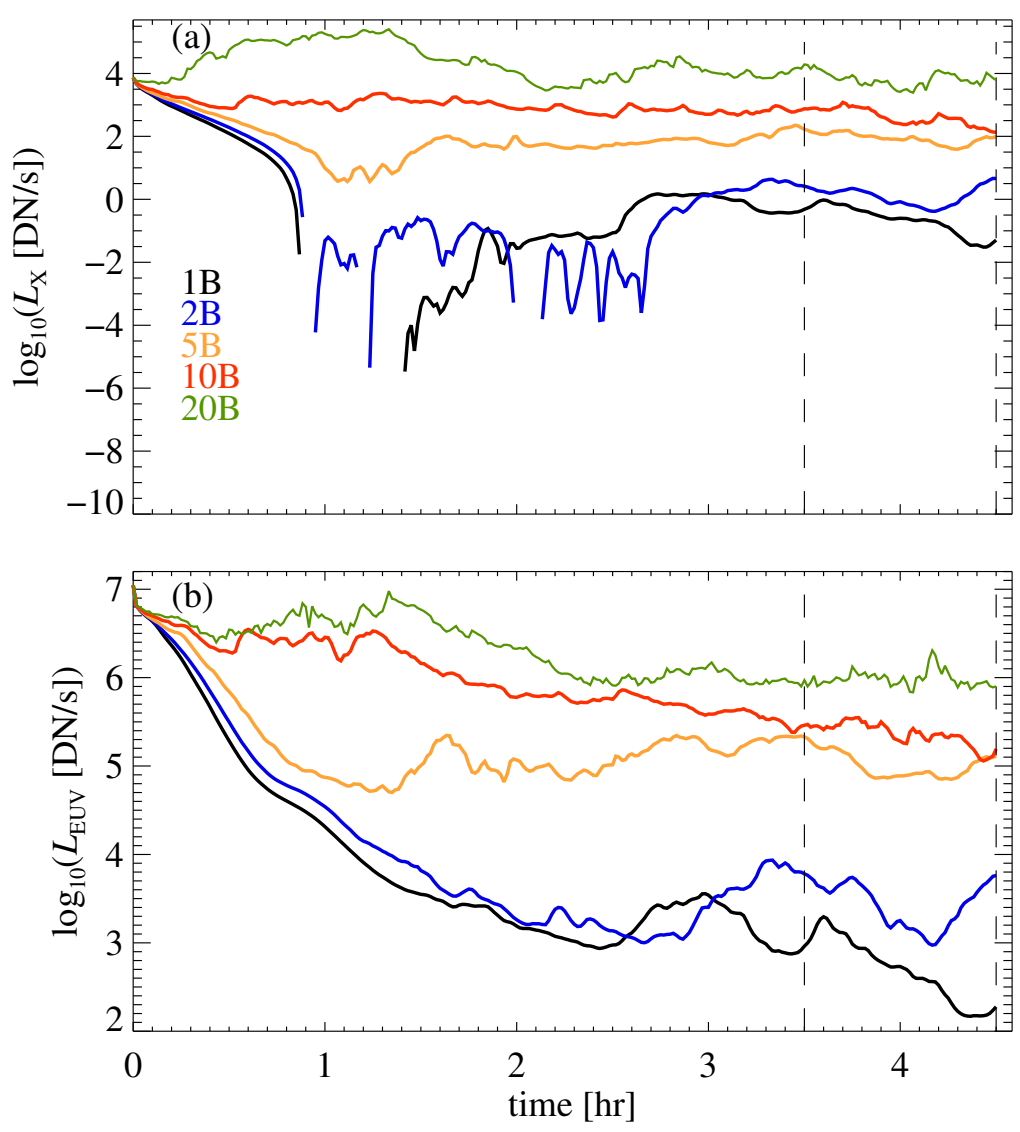

Figure 5.6: Temporal evolution of the coronal emission from integrated over the whole computational domain. Panel (a) shows the X-ray emission as seen by XRT in the Al-poly filter, panel (b) the EUV emission as it would be seen by AIA in the $171 \AA$ channel. The vertical dashed lines indicate the time span used for time averaging. The colors represent the different runs as indicated in the legend (cf. Table 5.1). See Sec. 5.4.4.

\subsection{Scaling relations in numerical experiments}

To characterize the model runs with different magnetic activity, i.e. unsigned magnetic surface flux, we investigate the scaling relations in the form of power laws between different parameters. In Sect. 5.4 we showed that the enhancement of the photospheric magnetic flux lead to a substantial increase of temperature, density, Poynting flux and coronal emission. In this section, we discuss the power-law relations of various quantities averaged in space and time quantities. We will discuss these results in Sect. 5.6 including the study of Zhuleku et al. (2020).

We first concentrate the relation of the the vertical component of the average Poynting flux $\left\langle S_{z}\right\rangle$ to the unsigned surface magnetic flux $\Phi$ and the averaged total coronal heating $\left\langle H_{\text {tot }}\right\rangle$ (see Fig. 5.7). Each cross in the two figures represent averaged values of the respective quantities in each individual numerical model (cf. Table 5.1). Here we take the the horizontal average of the Poynting flux $\left\langle S_{z}\right\rangle$ at the height where the horizontally averaged temperature is $10^{5} \mathrm{~K}$, i.e. the base of the corona and average it in time from 3.5 to $4.5 \mathrm{hr}$ (see Sect. 5.4.4). This represents the energy flux (per unit area) into the corona. The total 
averaged coronal volumetric heating $\left\langle H_{\text {tot }}\right\rangle$ is calculated according to Eq. (5.17) and then averaged between times 3.5 and $4.5 \mathrm{hr}$ as discussed in Sect. 5.4.4. The unsigned surface flux $\Phi$ is the integral over the bottom boundary, i.e. the stellar surface which is constant in time. The bars in Fig. 5.7 indicate the standard deviation of the respective quantity in time. For relations displayed in Fig. 5.7 we perform power-law fits (indicated by the red line) that result in

$$
\begin{array}{lll}
\left\langle S_{z}\right\rangle \propto \Phi^{\beta} & \text { with } & \beta=1.71 \pm 0.42, \\
\left\langle S_{z}\right\rangle \propto\left\langle H_{\text {tot }}\right\rangle^{\Gamma} & \text { with } & \Gamma=0.88 \pm 0.22 .
\end{array}
$$

Here $\Gamma$ corresponds to $1 / \gamma$ from the analytical model in Eq. (5.6) and Zhuleku et al. (2020). The two scalings (5.18) and (5.19) imply that the heating increases roughly quadratically with magnetic flux, $\left\langle H_{\text {tot }}\right\rangle \propto \Phi^{1.94}$.

As a next step, we relate the coronal temperature $T$ and density $\rho$ to the total coronal heating $\left\langle H_{\mathrm{tot}}\right\rangle$. Here we test to what extent the coronal temperature and density in our numerical model deviates from the analytic RTV scaling laws as given in Eqs. (5.1) and (5.2). For this, we calculate the average temperature $\langle T\rangle$ and density $\langle\rho\rangle$ in the corona in a height range from $z=10 \mathrm{Mm}$ to $20 \mathrm{Mm}$ for each of the models. We choose this particular height range because here the bright structures appear (see Fig. 5.2). If we would also include higher regions of the box, the averages would no longer represent the actually visible parts of the corona. In addition, we average $\langle T\rangle$ and $\langle\rho\rangle$ in time from 3.5 to $4.5 \mathrm{hr}$ as discussed in Sect. 5.4.4. We show the corresponding plots including the power-law fits of $\langle T\rangle$ and $\langle\rho\rangle$ as a function of the total coronal heating $H_{\text {tot }}$ in Fig. 5.8. The power-law fits yield

$$
\begin{array}{lll}
\langle T\rangle \propto\left\langle H_{\text {tot }}\right\rangle^{a} & \text { with } & a=0.24 \pm 0.03, \\
\langle\rho\rangle \propto\left\langle H_{\text {tot }}\right\rangle^{b} & \text { with } & b=0.76 \pm 0.05 .
\end{array}
$$

Finally, we address the relation of the X-ray emission to the heating rate and the unsigned surface magnetic flux. As the photospheric magnetic flux is larger for models with higher magnetic activity, also the total dissipated energy is larger. And because the $\mathrm{X}$-ray emission is expected to increase with the heating rate, it should also be larger for higher unsigned magnetic flux. For the analysis we consider the averaged emission, $\left\langle L_{\mathrm{X}}\right\rangle$, that is integrated over whole computational domain and averaged as the other quantities from 3.5 to $4.5 \mathrm{hr}$. The corresponding relations are plotted in Fig. 5.9 and Fig. 5.10a, and the power-law fits give

$$
\begin{array}{ll}
\left\langle L_{\mathrm{X}}\right\rangle \propto \Phi^{m} & \text { with } \quad m=3.44 \pm 0.28, \\
\left\langle L_{\mathrm{X}}\right\rangle \propto\left\langle H_{\text {tot }}\right\rangle^{q} & \text { with }
\end{array}
$$

We apply the same integration and time averaging to the EUV emission as seen by AIA in its $171 \AA$ A channel. Its relation to the heat input is displayed in Fig. $5.10 \mathrm{~b}$ and the powerlaw fit reveals an almost linear relation,

$$
\left\langle L_{\mathrm{EUV}}\right\rangle \propto\left\langle H_{\mathrm{tot}}\right\rangle^{p} \quad \text { with } \quad p=1.05 \pm 0.09 .
$$



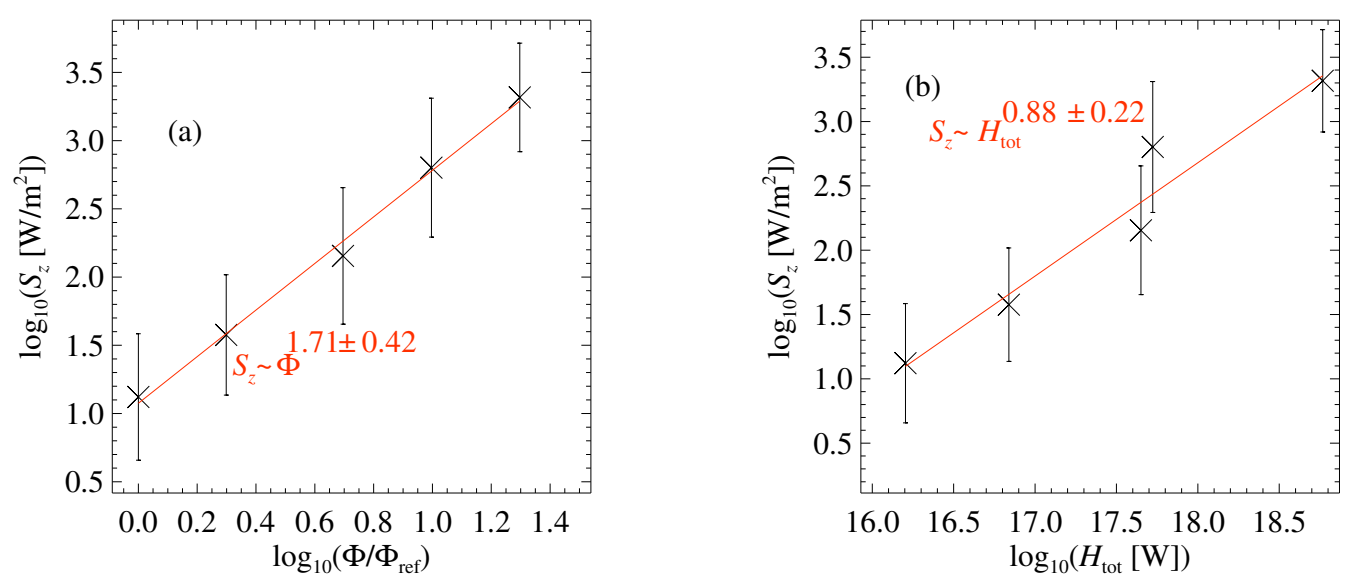

Figure 5.7: Scaling of average Poynting flux $\left\langle S_{z}\right\rangle$ with unsigned surface flux $\Phi$ and average coronal heating $\left\langle H_{\text {tot }}\right\rangle$. Each data point represents an average for one of the model runs with different unsigned surface magnetic flux as listed in Table 5.1. As a reference for the magnetic flux, $\Phi_{\text {ref }}$, we choose the magnetic flux of the least active setup, run 1B (cf. Table 5.1). The bars represent the standard deviation of $S_{z}$ in time. The red lines are power-law fits to the data. See Sects. 5.5 and 5.6.1.

\subsection{Discussion}

\subsubsection{Energy input into the corona}

The solar coronal heating problem and the underlying physical mechanism has been extensively discussed the last 70 years. Two of the main mechanisms that are being considered are the Alfvén-wave model (e.g. van Ballegooijen et al. 2011) and the field-line braiding or nanoflare model (Parker 1972, 1983). These two processes have a different dependence of the Poynting flux, and hence the heat input, on the magnetic field. A simple estimate for these dependencies has been given by Fisher et al. (1998). Here we follow their arguments. For an Alfvén wave with constant amplitude, the Poynting flux will be proportional to the propagation speed, i.e. the Alfvén velocity, and hence to the magnetic field $B, S_{z} \propto B$. For the field-line braiding, the Poynting flux will be set by the driving motions with speed $u$ and the magnetic field, $S \propto u \times B \times B$, and hence the scaling would be $S_{z} \propto B^{2}$ (assuming that the driving motions do not change with $B$ ).

In our numerical experiments we find a power-law scaling between Poynting flux and (unsigned) magnetic flux with a power-law index of about $1.7 \pm 0.4$, see Eq. (5.18) and Fig. 5.7a. Within the uncertainties this is consistent with the above (analytical) estimate of 2. Of course, this is not surprising, because our in our model we drive the coronal magnetic field through footpoint motions consistent with the field-line braiding scenario. Still, it is reassuring to recover this scaling by our numerical model.

Observationally it is clear that Alfvén waves are present in the corona (e.g. Tomczyk et al. 2007). Still, it remains unclear if the energy they carry would be sufficient to energize the corona. Waves might play a role in the quiet Sun corona, but they seem to not be able to heat active regions (McIntosh et al. 2011). Our particular model can contribute little to this discussion, because we do not fully resolve Alfvén waves, which is because of the 

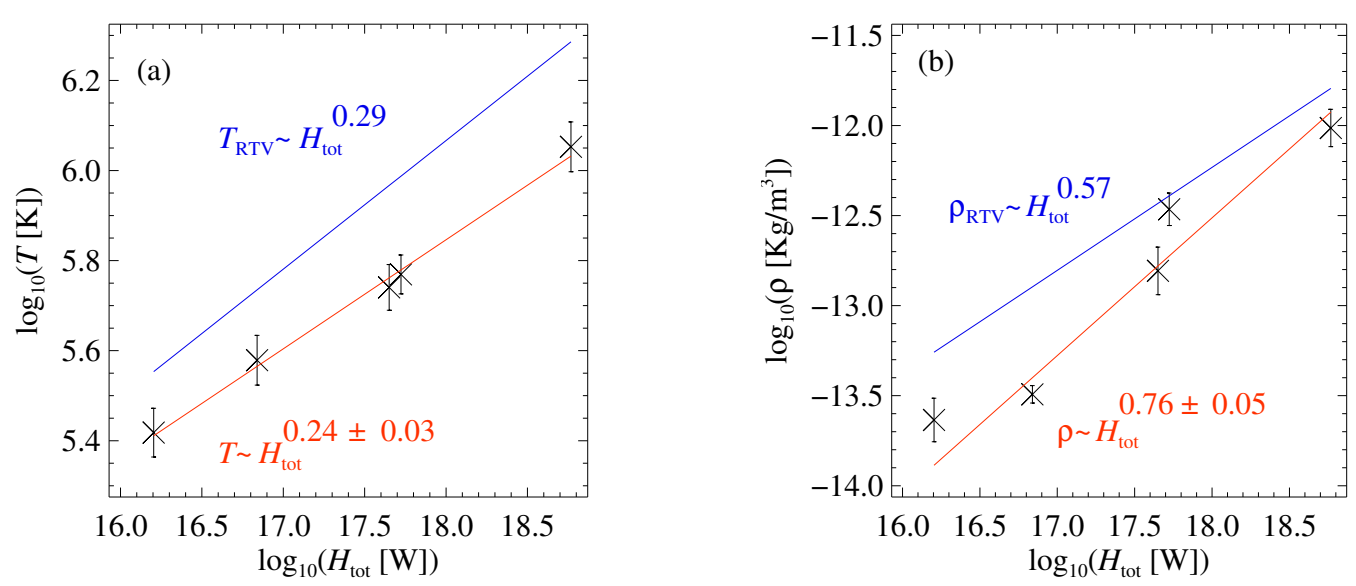

Figure 5.8: Scaling of average temperature $\langle T\rangle$ and density $\langle\rho\rangle$ with average coronal heat input $\left\langle H_{\text {tot }}\right\rangle$. Each data point represents an average for one of the model runs with different unsigned surface magnetic flux listed in Table 5.1. The bars represent the standard deviation of the spatial averages of $T$ and $\rho$ in time. The red lines are power-law fits to the data. The blue lines indicate what is expected from the RTV scaling laws. See Sects. 5.5 and 5.6.2.

comparably large dissipation.

Finally, we expect that the total energy dissipated in the coronal volume matches the Poynting flux at the base of the corona. In this case the Poynting flux should scale linearly with the total amount of energy dissipated in the corona. In our numerical experiments we find a power-law relation with a power-law index of about $0.9 \pm 0.2$, which is consistent with linear, see Eq. (5.19) and Fig. 5.7b.

\subsubsection{RTV scaling laws compared to numerical experiments}

One obvious check for the numerical experiments is to what extent the averaged quantities will follow the RTV scaling laws Eqs. (5.1) and (5.2). Of course, because of the spatial and temporal variability we cannot expect a perfect match, but the average quantities should roughly follow these scalings, as it was found in an earlier model for one single (small) solar active region (Bourdin et al. 2016).

The original RTV scalings in Eqs. (5.1) and (5.2) include a dependence on the loop length $L$. In our numerical models, however, the physical size of the computational box is kept constant. Therefore, the coronal loops can be considered to have similar lengths. Consequently, in this study we only have to consider the dependence of temperature and density on (total) heating in the coronal volume, $H_{\text {tot }}$.

For a comparison to the RTV scalings one should not only compare the power/law indices of the scaling, but also the absolute values of the temperature $T$ and density $\rho$ as given in the original paper by Rosner et al. (1978). To calculate the predictions from the RTV scalings, i.e. $T_{\mathrm{RTV}}$ and $\rho_{\mathrm{RTV}}$, we use a loop length of $L=30 \mathrm{Mm}$, which is similar to the average loop length we find in the emission patterns synthesized from the model (see Fig. 5.2). For the volumetric heating rate we use the (total) heating in the coronal volume, $\left\langle H_{\text {tot }}\right\rangle$, divided by the coronal volume, which gives the (average) volumetric heating. The 


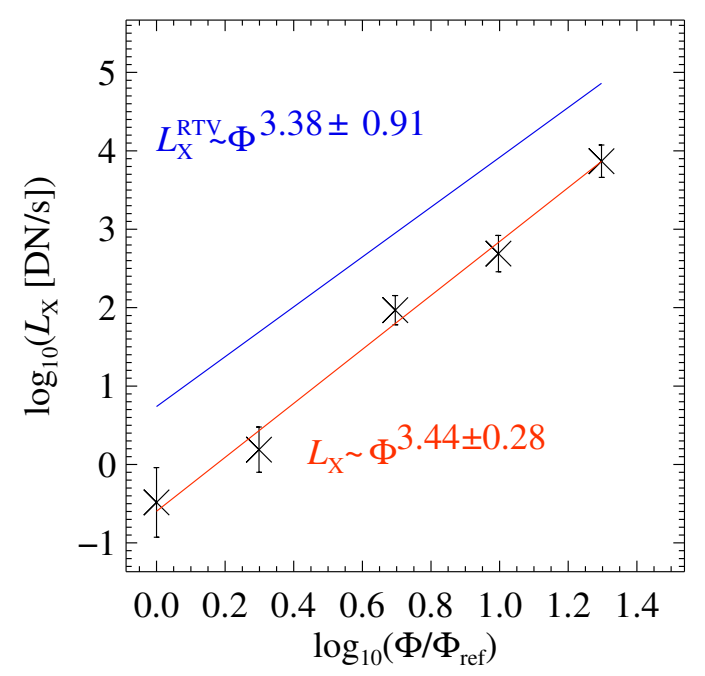

Figure 5.9: Scaling of average X-ray emission $\left\langle L_{X}\right\rangle$ with unsigned surface flux $\Phi$. Each data point represents an average for one of the model runs with different unsigned surface magnetic flux listed in Table 5.1. As a reference for the magnetic flux, $\Phi_{\text {ref }}$, we choose the magnetic flux of the least active setup, run $1 \mathrm{~B}$ (cf. Table 5.1). The bars represent the standard deviation of $L_{X}$ in time. The red lines are power-law fits to the data. The blue lines show the analytic power-law relations based on the RTV scaling laws. See Sects. 5.5, 5.6.3 and 5.6.4.

resulting variation of $T_{\mathrm{RTV}}$ and $\rho_{\mathrm{RTV}}$ with $\left\langle H_{\mathrm{tot}}\right\rangle$ is shown in Fig. 5.8 (blue lines). Obviously, the power law-indices of 0.29 and 0.57 for these correspond to $2 / 7$ and $4 / 7$ in Eqs. (5.1) and (5.2).

The power-law relation of the average temperatures $\langle T\rangle$ and density $\langle\rho\rangle$ in the numerical models with $\left\langle H_{\text {tot }}\right\rangle$ are close to what is expected from RTV. However, both the temperature and the density are significantly lower, by about a factor 2 and 3, see Fig. 5.8 and Eqs. (5.20) and (5.21).

The reason for this underestimation is mainly due to the averaging process of the temperature and density. The bright loops are hotter and denser than the ambient corona so that the averages underestimate the values corresponding to the RTV scaling relations. Still, we conclude that the numerical models and the analytical scaling relations are consistent (as an order-of-magnitude estimation).

\subsubsection{Relation of X-ray emission to surface magnetic flux}

The central result of our study is the power-law relation between the synthetic X-ray emission $L_{\mathrm{X}}$ and the unsigned surface magnetic flux $\Phi$. We show this relation $L_{\mathrm{X}} \propto \Phi^{m}$ with a power-law index $m$ of about 3.4 \pm 0.3 in Fig. 5.9, see also Eq. (5.22).

This $L_{\mathrm{X}}$ vs. $\Phi^{m}$ relationship has been extensively discussed in numerous observational studies of the Sun and of stars of different spectral types (see e.g. Fisher et al. 1998; Pevtsov et al. 2003; Vidotto et al. 2014). However, the physical reasons behind the observed power-law relation remained unclear. Observations of various solar features and solar-like stars suggest a relation close to but slightly steeper than linear, i.e. $m \gtrsim 1$ (e.g. 

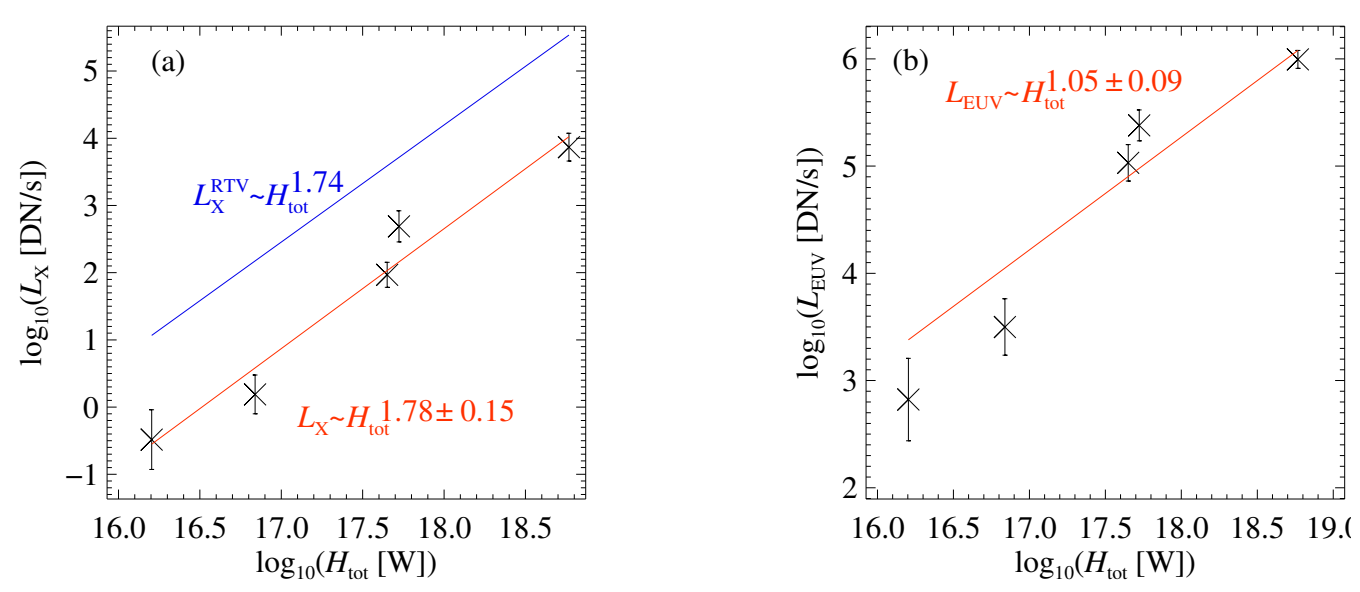

Figure 5.10: Scaling of average X-ray and EUV emission, $\left\langle L_{X}\right\rangle$ and $\left\langle L_{\mathrm{EUV}}\right\rangle$ with average coronal heating $\left\langle H_{\text {tot }}\right\rangle$ for model runs listed in Table 5.1. The bars represent the standard deviation of $L_{\mathrm{X}}$ and $L_{\mathrm{EUV}}$ in time. The red lines are power-law fits to the data. The blue line in panel (a) indicates what is expected from the RTV scaling laws. See Sects. 5.5 and 5.6.5.

Fisher et al. 1998; Pevtsov et al. 2003). More recent studies find the power-law to be close to quadratic ( $m=1.8$; Vidotto et al. 2014) or even steeper $(m=2.68$; Kochukhov et al. 2020). While Kochukhov et al. (2020) considered stars similar to the Sun in terms of spectral type, all these were considerably more active than the Sun in terms of X-ray luminosity. The large sample of Vidotto et al. (2014) also contained mainly stars being significantly more active than the Sun.

An almost linear relation of $L_{\mathrm{X}}$ vs. $\Phi$ could be simply understood by increasing the number of active regions on a (solar-like) star. If the filling factor of active regions is low and one simply doubles the number of active regions, both the total unsigned magnetic flux on the stellar surface and the X-ray emission would double. Hence the linear relation between $L_{X}$ and $\Phi$. This, in principle, could explain the findings of Fisher et al. (1998) and Pevtsov et al. (2003).

Active stars show X-ray luminosities (compared to their bolometric luminosity) that can be three or more orders of magnitude larger than that of the Sun (e.g. Vidotto et al. 2014). In this case there would be simply not enough space on the star to cover it with enough (solar-like) active regions. This means, the X-ray emission per active region has to increase. This is exactly what we find in our model when increasing the magnetic flux of an active region while keeping its size (i.e. area) the same. This leads to a steep increase of X-ray luminosity with surface magnetic flux, an increase that is even steeper than observed: the power-law index $m$ for $L_{X} \propto \Phi^{m}$ is about 3.4 in our model, while the largest value found in observations is below 2.7 (Kochukhov et al. 2020). This overestimation of the power-law index by our model indicates that on real stars we might find a mixture of an increase of the numbers of active regions that goes together with the increase of the peak magnetic field strength (or magnetic flux per active region) for more active stars. 


\subsubsection{Analytical model for scaling of X-ray emission}

We will now compare the scaling of $L_{\mathrm{X}} \propto \Phi^{m}$ to some basic analytical considerations. In an earlier study we derived an analytical scaling of the X-ray emission with surface magnetic flux that is based on the RTV scaling relations (Zhuleku et al. 2020). As discussed in the summary of that model in Sect.5.2, there we used a scaling of active region size with (unsigned) magnetic flux based on solar observations, see Eq. (5.7). In contrast, in our numerical model we keep the size or area covered by the active region constant, hence here the power-law index in Eq. (5.7) is $\delta=0$. This simplifies our analytical scaling from Eq. (5.3) to

$$
m=\frac{\beta \gamma}{7}(2 \alpha+8)
$$

According to Eq. (5.4), $\alpha$ is the power-law-index relating the temperature response (or contribution) function to temperature. Here we approximate this for X-rays as seen by the XRT on Hinode (see Fig. 5.3), where a power-law fit yields

$$
R_{\mathrm{X}} \propto T^{\alpha} \quad \text { with } \quad \alpha=2.1 \pm 0.2
$$

We give a more extensive discussion on the temperature responses for different instruments in Zhuleku et al. (2020).

The other two parameters $\beta$ and $\gamma$ in Eq. (5.25) we take from the relations of the Poynting flux to the unsigned surface magnetic flux and the total heating in Eqs. (5.18) and (5.19), with $\gamma=1 / \Gamma$ in Eq. (5.19). This results in the analytic scaling (based on RTV) of

$$
L_{\mathrm{X}}^{\mathrm{RTV}} \propto \Phi^{m^{\prime}} \quad \text { with } \quad m^{\prime}=3.38 \pm 0.91 .
$$

This is overplotted onto the results from the numerical experiments in Fig. 5.9 as a blue line. We conclude that this power-law index is in good agreement with the value we obtained from the numerical models, see Eq. (5.22) and Fig. 5.9.

Just as for the comparison to the RTV scalings in Sect. 5.6.2, also here it is not sufficient to find a match of the power law index, but also the absolute values of the derived X-ray emission have to be of the same order for a good match. To get the constant of proportionality in Eq. (5.27), we have to assign a volume of the emitting structure described in the analytical scaling. For the comparison to our numerical model we thus assign the volume of the loop(s) dominating the coronal emission. Using the loops in Fig. 5.2 as a guideline, these have a length and a radius of about $L \approx 30 \mathrm{Mm}$ and $r \approx 2.5 \mathrm{Mm}$. With the volume of $V=\pi R^{2} L$ we then find the total $\mathrm{X}$-ray radiation from the loops based on the (RTV) scaling relations of $L_{\mathrm{X}}^{\mathrm{RTV}}$. Within an order of magnitude these match what we find in the numerical study. The deviation is mainly because of the factor of about 3 difference in density (cf Sect. 5.6.2) which enters the emission quadratically.

\subsubsection{X-ray and EUV emission versus coronal energy input}

Common sense suggests that a higher energy input into the corona should result in a higher X-ray emission. Indeed, this is what we find in Fig. 5.10a. However, we find an almost quadratic relationship, $\left\langle L_{\mathrm{X}}\right\rangle \propto\left\langle H_{\text {tot }}\right\rangle^{1.8}$, cf. Eq. (5.24). This non-linearity is counter-intuitive: Assuming that the plasma in our numerical model has reached a relaxed 
state, then whatever energy reaches the corona should be radiated away as X-ray emission (i.e., naively, we would expect a linear dependence).

This (roughly) quadratic relation can be understood when going back to the RTV scalings. The X-ray emission is given by $L_{\mathrm{X}} \propto n^{2} R_{\mathrm{X}}$. Combining this with the fit to the X-ray response function in Eq. (5.26) and the RTV scaling relations Eqs. (5.1) and (5.2) provides the analytical scaling for the X-ray emission with (average) coronal heat input,

$$
L_{\mathrm{X}}^{\mathrm{RTV}} \propto\left\langle H_{\mathrm{tot}}\right\rangle^{q^{\prime}} \text { with } \quad q^{\prime}=1.74 \pm 0.58 .
$$

This is shown in comparison to the results from the numerical model in Fig. 5.10a as a blue line. As before, we assumed a constant loop length, because the active region size is the same for all the numerical experiments.

This consideration provides an understanding of the non-linear relation between Xray output and heat input. In the corona the energy input will be only partly balanced by the X-ray output (also heat conduction, a wind outflow and radiation at other wavelengths will play a role). Because the X-ray output shows a non-linear temperature dependence, naturally, it will also be connected in a non-linear fashion to the heat input. The simple RTV scaling relations together with the temperature response of X-ray emission give rise to the roughly quadratic dependence of X-rays on heat input.

The situation changes when investigating another wavelength region, which essentially implies to probe a different temperature range. For this we use the channel as seen by AIA at $171 \AA$. The EUV emission in this band is mostly originating from temperatures around $1 \mathrm{MK}$ (cf. Fig. 5.3). If we apply the same analysis as for the X-ray emission, the numerical experiments show an almost linear scaling with heat input, $\left\langle L_{\mathrm{EUV}}\right\rangle \propto\left\langle H_{\mathrm{tot}}\right\rangle^{1.1}$, see Fig. 5.10b. The reason this relation is less steep than for the X-rays is because of the different temperature response in the EUV.

The response in the EUV cannot be simply approximated by a power law (see Fig. 5.3). However, over the temperature range of most our models, as a very rough zeroth order approximation we could assume the response in the EUV channel to be constant. This would be consistent with the power-law index for the response function, i.e. $\alpha$ in Eq. (5.4), to be zero. Following the above procedure for the X-rays, in analogy to Eq. (5.28) we would find $L_{\mathrm{EUV}}^{\mathrm{RTV}} \propto\left\langle H_{\text {tot }}\right\rangle^{1.14}$ for the EUV emission. This result compares well with the numerical experiments (cf. Fig. 5.10b), but certainly it has to be taken cum grano salis, which is because of our very rough assumption of $\alpha=0$. This is why we do not overplot an analytical approximation in Fig. 5.10b as we did for the other scaling relations. Still, this consideration underlines the importance of the temperature response for establishing a relationship between coronal emission and heat input.

\subsubsection{Relating the X-rays the EUV emission}

Finally, we briefly investigate the scaling relation between the X-ray and the EUV emission. Solar and stellar studies have addressed the relation between the radiative fluxes in different wavelength bands, also called flux-flux-relations. One prominent example is the relation between the coronal $\mathrm{X}$-ray radiative flux and the radiance in the chromospheric CaII line with $F_{\mathrm{X}} \propto\left(F_{\text {CaII }}\right)^{1.67}$ (e.g. Schrijver 1983). Power-law relations have also been derived with other chromospheric and transition region lines, e.g. MgII, SiII, CII, and CIV (Schrijver 1987). The higher the temperature in the source region of the emission, 


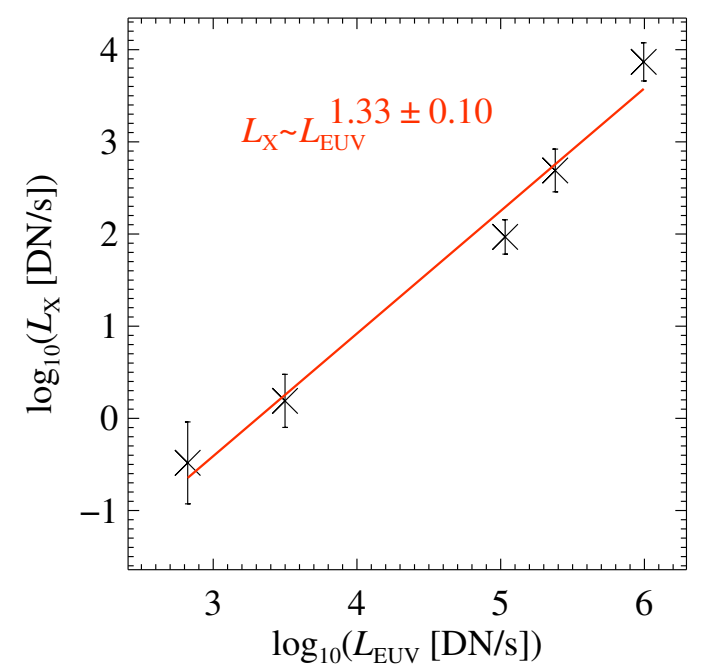

Figure 5.11: Scaling of averages of X-ray emission $\left\langle L_{X}\right\rangle$ with EUV emission $\left\langle L_{\mathrm{EUV}}\right\rangle$ for model runs listed in Table 5.1. The bars represent the standard deviation of $L_{\mathrm{X}}$ in time. The red line is a power-law fits to the data. See Sect. 5.6.6

the steeper the power-law relation of the respective line to the surface magnetic field (e.g. Testa et al. 2015). This implies that the relation of $\left\langle L_{X}\right\rangle$ vs. $\left\langle L_{\mathrm{EUV}}\right\rangle$ is less steep than the relation of X-rays to CaII.

In the numerical models we find that the relation between X-rays and the $1 \mathrm{MK}$ EUV emission follows a power law with about $\left\langle L_{\mathrm{X}}\right\rangle \propto\left\langle L_{\mathrm{EUV}}\right\rangle^{1.3}$, see Fig. 5.11. This is also consistent with other numerical studies (see e.g. Warnecke and Peter 2019b, their Fig. $10 \mathrm{~b})$. So indeed, as expected, it is less steep than X-rays to CaII with a power-law index about 1.7 .

Our numerical models are not realistic enough when it comes to the cooler parts of the atmosphere, in the transition region around $10^{5} \mathrm{~K}$ and the in particular in the cooler chromosphere. Still we see the right trend for the flux-flux relations and it will be left for future more realistic models to fully explore the flux-flux relations.

\subsection{Conclusions}

We performed a series of 3D MHD numerical simulations of active regions. With this we address the question how the (X-ray) emission from stellar coronae scales with the surface magnetic flux. Observationally this power-law scaling $L_{X} \propto \Phi^{m}$ is well established with the most recent studies giving power-law indices $m$ in the range of below 2 to almost 3 . (Vidotto et al. 2014; Kochukhov et al. 2020). So far, the physical basis for this relation is poorly understood in terms of (numerical) models.

For our model series we assumed that the area covered by the active region remains the same, and we changed the peak (or average) vertical field strength $B$ to change the (unsigned) magnetic flux at the surface by a factor of 20. This resulted in a change of the X-ray emission by more than four orders of magnitude. The scaling we found in our numerical experiments is a power law with an index $m \approx 3.4$, i.e. a bit steeper than found 
in observations. As we discussed in Sect. 5.6.3, this difference can be understood if on the real star there are not only active regions with higher magnetic flux (but the same area), but if there is also a larger filling factor of active regions, or in other words a larger number of active regions.

The results of our numerical experiments are consistent with an analytical scaling model (Zhuleku et al. 2020). This is based on the RTV scaling laws connecting the temperature and density to the heating rate and size of the coronal structure and the temperature response of the wavelength band the observations are performed. With this we have a clear understanding how and why the radiative X-ray output changes in a non-linear fashion in response to the heat input, and hence the surface magnetic flux.

In our numerical model we choose the specific approach to increase the surface magnetic flux by increasing the field strength. This is motivated by the very high field strengths seen on active M dwarfs of up to $8 \mathrm{kG}$ on average (e.g. Reiners 2012). The average being so large suggests that the highest field strengths on these stars could be even higher, maybe consistent with our model. Still, to investigate further possibilities we will study the response of the coronal emission to an increase of the magnetic flux by increasing the area of the active region(s) in a future project.

Of course our models for individual active regions cannot be expected to fully account for all aspects of the scaling of coronal emission with (unsigned) surface magnetic flux. Still, our approach indicates a way to understand the excessive increase of the observed $\mathrm{X}$-ray emission by 4 orders of magnitude from solar-type activity to fast rotating active stars (by e.g. Pizzolato et al. 2003; Vidotto et al. 2014). 


\title{
6 Influence of the size of the active region on the coronal $\mathrm{X}$-ray emission
}

\begin{abstract}
*Chapter 6 follows the paper Influence of the size of the active region on the coronal X-ray emission by J. Zhuleku, J. Warnecke and H. Peter. This paper is in preparation and it will be submitted in spring of 2021. I performed all the simulations, analysed and interpreted the data and wrote the first draft of the paper.
\end{abstract}

\subsection{Introduction}

Stars more active than the Sun are observed to have a much higher coronal X-ray emission, even a thousand times higher than the Sun. The high level of X-ray emission observed on active stars depends on age and thus on the rotation rate (Pizzolato et al. 2003). A higher rotation rate is responsible for a more efficient dynamo action which produces a higher heat input and provides the necessary energy to heat the corona to high temperature. As a reference, the solar corona could reach roughly $10 \mathrm{MK}$, whereas the more active stars could reach even higher temperatures. Because of these high temperatures, stars exhibit a strong X-ray emission which can be used as a diagnostic to study the stellar coronae.

The main focus of this chapter is the study of the relationship between the coronal $\mathrm{X}$-ray emission $L_{\mathrm{X}}$ and the surface magnetic flux $\Phi$. This relationship is found to follow a well-known power-law, $L_{\mathrm{X}} \propto \Phi^{m}$. Older studies found the power-law index $m$ to be close to unity (Fisher et al. 1998; Pevtsov et al. 2003). More recent studies, on the other hand, place this index to larger values. More precisely, Vidotto et al. (2014), studying a sample of stars more active than the Sun, found this index to be almost quadratic (i.e. $m=1.8$ ). Furthermore, Kochukhov et al. (2020) using a sample of $\mathrm{G}$ type stars but more active than our Sun found this index to be even steeper (i.e. $m=2.68$ ). The wide range of the power-law index $m$ found in observations is indicative of our poor understanding of this relation.

The $L_{\mathrm{X}} \propto \Phi^{m}$ relation can be modeled as described in Zhuleku et al. (2020). In that study, we found that using the RTV scaling laws (Rosner et al. 1978) along with other relations can lead to a single analytic expression relating the X-ray emission $L_{\mathrm{X}}$ to the surface magnetic flux $\Phi$. We found that mainly three parameters have a significant effect on the power-law index $m$. The first two parameters (namely $\alpha$ and $\beta$ ) are related to the instruments temperature response function and the heating mechanism responsible for the coronal heating (for more details see Zhuleku et al. 2020). The last parameter characterizes the amount of surface area $A$ of a star or the Sun covered by a magnetic field (e.g. active region). It is parameterized by the parameter $\delta$ and it also follows a 
Table 6.1: Summary of the numerical runs. The second column represents the size of the numerical box. Third column indicates the resolution of each run. $\Phi$ is the total surface unsigned magnetic flux.

\begin{tabular}{cccc}
\hline \hline Run & Volume $\left[\mathrm{Mm}^{3}\right]$ & Resolution (grid points) & $\Phi[\mathrm{Mx}]$ \\
\hline R25 & $25 \times 25 \times 25$ & $64 \times 64 \times 64$ & $9.4 \times 10^{20}$ \\
R50 & $50 \times 50 \times 50$ & $128 \times 128 \times 128$ & $3.7 \times 10^{21}$ \\
R75 & $75 \times 75 \times 75$ & $192 \times 192 \times 192$ & $8.1 \times 10^{21}$ \\
R100 & $100 \times 100 \times 100$ & $256 \times 256 \times 256$ & $1.5 \times 10^{22}$ \\
R200 & $200 \times 200 \times 200$ & $512 \times 512 \times 512$ & $6.0 \times 10^{22}$ \\
\hline
\end{tabular}

power-law $A \propto \Phi^{\delta}$. Solar and stellar studies suggest $\delta=0.82$. The value of $\delta$ is essential for understanding how the power-law index $m$ varies for other stars.

For stars where their entire surface is covered with a magnetic field implies that $\delta=$ 0 . Therefore, to increase the surface magnetic flux, the star has to increase the overall strength of the surface magnetic field while the surface area remains unchanged. Our analytical model suggests a steep value for the power-law index $m$ which contradicts some of the earlier observational studies. To test this particular behavior using realistic numerical models, we presented in Chap. 5 a series of 3D MHD simulations for the case of $\delta=0$. The magnetic activity was increased by increasing the strength of the vertical surface magnetic field $B_{z}$ at the photosphere. We showed that the power-law index $m$ is steep compared to observations (see e.g. Fisher et al. 1998; Pevtsov et al. 2003). However, the results are in good agreement with the theoretical predictions proposed by Zhuleku et al. (2020). We suggest that stars change not only the peak strength of the surface magnetic field but also the number of active regions. Along with the recent studies of Vidotto et al. (2014), and Kochukhov et al. (2020) our results can explain the increase of the X-ray emission found in more active stars than the Sun.

In this work, we examine the other extreme case (i.e. $\delta=1$ ). For this case, we again perform a series of 3D MHD numerical simulations. The size of the numerical box increases, covering a larger surface area while the absolute value of the surface magnetic field is kept constant. This will lead to an increase in the overall surface magnetic flux. For the rest of the chapter, we concentrate on the effect of the surface magnetic field distribution on coronal emission and coronal heating. In the end, we discuss scaling relations of the coronal X-ray emission as a function of the surface magnetic flux and how it compares with our analytical model and observations.

\subsection{Setup}

In this chapter, we investigate how the change in the horizontal spatial distribution of the surface magnetic field affects coronal X-ray emission. To study that, we scale the size of the numerical box by a constant factor to increase the surface area of the active region. This leads to an overall increase of the total surface unsigned magnetic flux $\Phi$. The peak strength of the magnetic field is kept constant at $5 \mathrm{kG}$. Therefore, the surface magnetic flux is proportional to the surface area $\Phi \propto A$. We perform a series of five numerical experiments. The first run, $\mathrm{R} 25$, represents a small active region inside a computational 
domain covering an area of $25 \times 25 \mathrm{Mm}^{2}$. The last run, R200, represents a large active region inside a computational domain of a 200x200 $\mathrm{Mm}^{2}$ (see Table 6.1). A change in the size of the numerical box increases the length of the coronal loops. That is to be expected since the increase of the computational box drags the opposite magnetic polarities of the active region further apart. The vertical extent of the numerical boxes for all the runs listed in Table 6.1 is increased by the same constant as in the horizontal direction.

The basic numerical setup is similar to the simulations presented in Chap. 5. Here we give a brief description of the model. The solar and stellar atmosphere is modeled in a $3 \mathrm{D}$ Cartesian box from the photosphere to the corona. For the numerical simulations, we use the solar coronal model based on Bingert and Peter (2011). We use the Pencil Code (Brandenburg et al. 2020) to solve the 3D MHD equations, namely the continuity, momentum, energy, and induction equations from the photosphere to the corona (see Chap.4). As a photospheric boundary condition for the vertical component of the magnetic field, we include an observed magnetogram of a solar active region (see Fig.1 of Chap. 5). A prescribed photospheric driver implemented at the bottom boundary will shuffle the magnetic footpoints of the active region. The photospheric driver mimics well the observed granular motion of the Sun (Gudiksen and Nordlund 2002; Bingert and Peter 2011). The coronal heating is provided by Ohmic dissipation of the induced currents due to the braided magnetic field lines. The energy will be radiated away through an optically thin radiative function. We furthermore include a realistic Spitzer heat conduction along the magnetic field lines (see also Chap. 4). The viscosity $v$ and resistivity $\eta$ are set to constant values through the whole box and are only reduced at the few grid points near the bottom boundary. To speed-up the simulations, a non-Fourier heat flux scheme together with the Boris correction is implemented in the model (see Warnecke and Bingert 2020, and Chap.4). The boundary, initial conditions, and all the physical parameters are similar to the ones used in Chap. 5 and a detailed description is discussed by Bingert and Peter (2011) and Warnecke and Bingert (2020). Finally, to keep the grid resolution of $390 \mathrm{~km}$, we scale the number of grid points in the same way as we scale the size of the numerical box. That would mean that if we double the size of the box, we also double the number of grid points.

\subsection{Results}

\subsubsection{Synthetic coronal emission and temporal evolution}

Coronal loops are considered to play an important role in the heating of the solar and stellar corona. Coronal loops in our Sun have been directly observed. However for other stars, this is not possible. Observations of stellar flares have shown that typical stellar loops can vary in length, and they can reach up to solar radius (Getman et al. 2008). Because of the high plasma temperature located in coronal loops, we observe them in the extreme ultraviolet (EUV) and X-ray regime. To synthesize the EUV emission from our numerical model we use the $171 \AA$ channel of the Atmospheric Imaging Assembly (AIA; Lemen et al. 2012) onboard the Solar Dynamic Observatory (SDO; Pesnell et al. 2012). As in Chap. 5, for the X-ray emission we use the Al-poly filter of the X-ray telescope (XRT; Golub et al. 2007b) onboard the Hinode (Kosugi et al. 2007). 

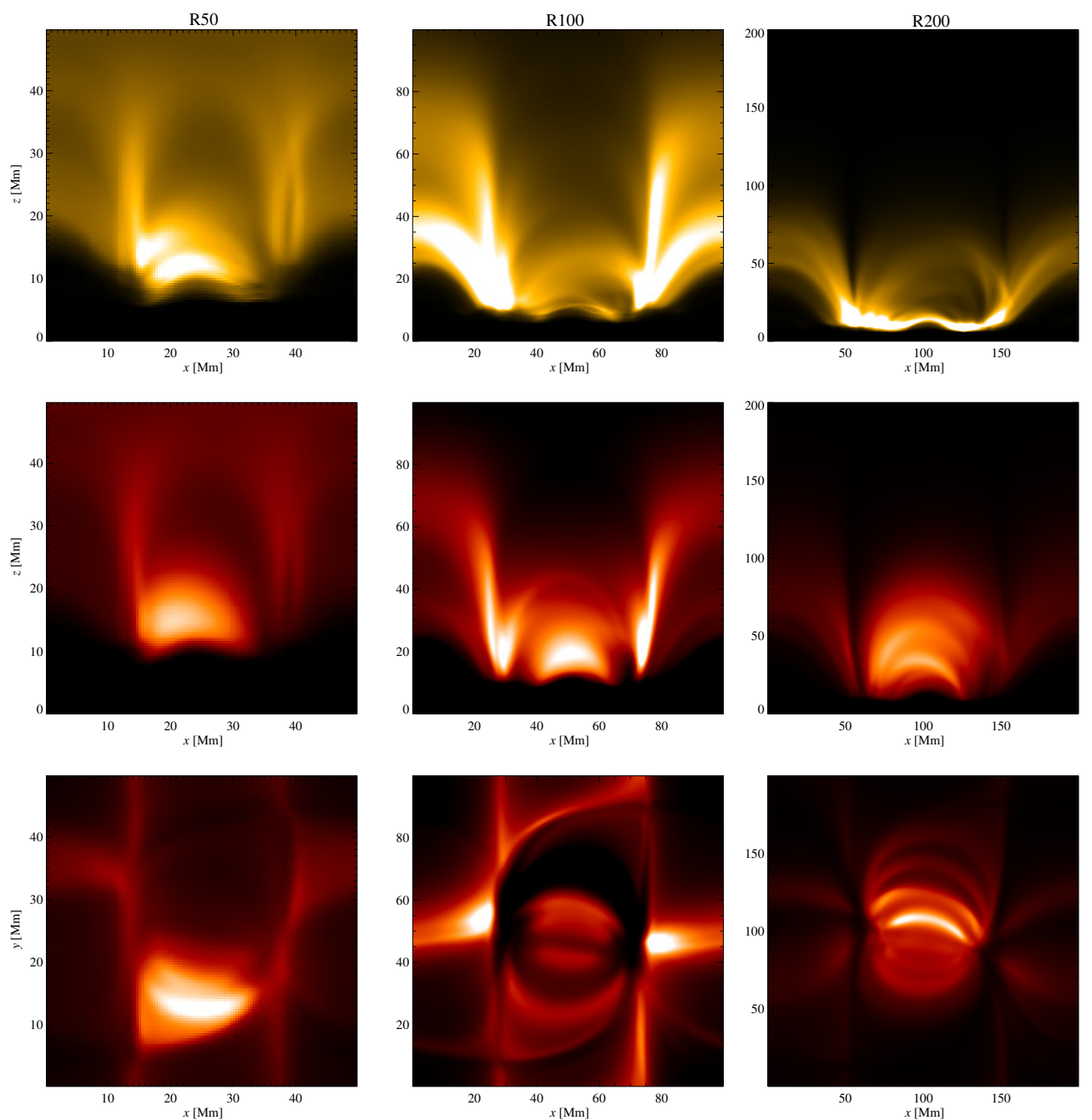

Figure 6.1: Synthetic EUV and X-ray emission for the runs R50, R100 and R200. The first row shows the synthesized EUV emission integrated in the $y$-direction, as it would have been observed from the AIA instrument for the $171 \AA$ channel. The second and third row show the integrated synthetic X-ray emission in the $y$ and $z$ direction respectively, as it would have been observed by the Al-poly filter of the XRT onboard Hinode. Both the EUV and X-ray emission are averaged in time during the relaxed phase. Each plot is scaled with the maximum EUV or X-ray emission respectively. For the larger box size coronal loops increase in length. 

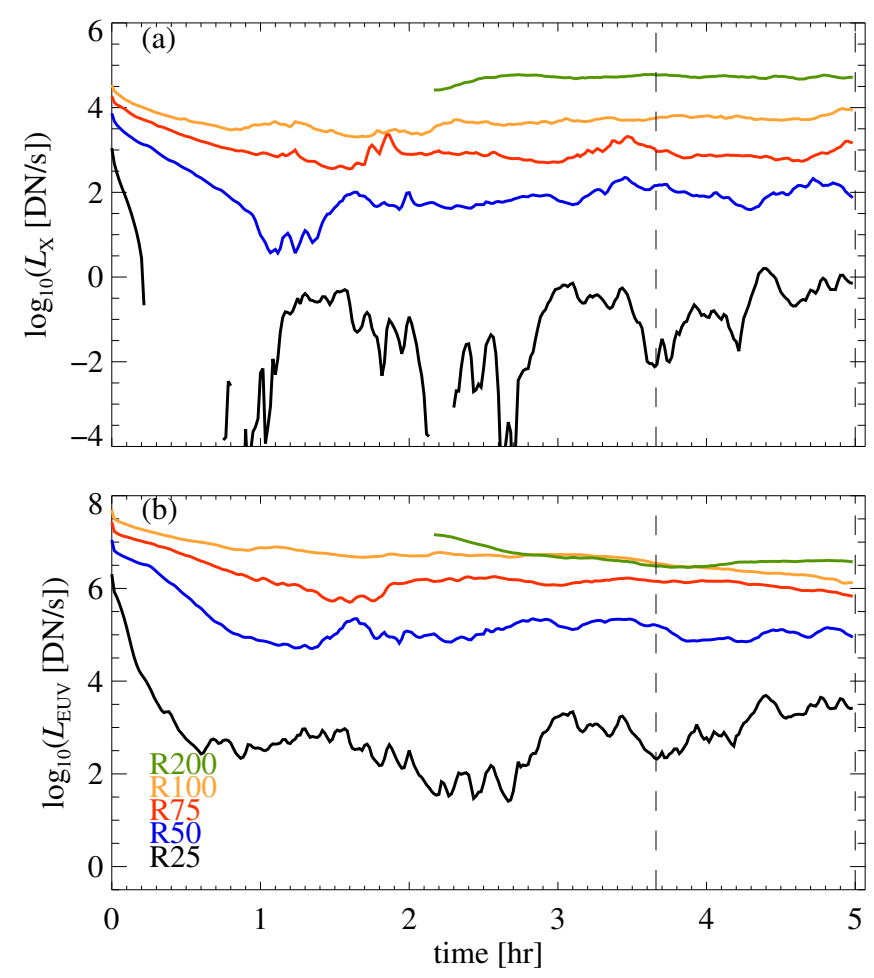

Figure 6.2: Time evolution of coronal EUV and X-ray emission. a) Total integrated X-ray emission over time for all the runs seen in Table 6.1. b) Total integrated EUV emission corresponding to the AIA 171 channel over time for all the runs. The vertical dashed lines indicate the relaxed phase used for the temporal averages.

As we discussed in Chap. 3 and Chap. 5, the optically thin radiation is proportional to electrons density squared times the temperature response function $R(T)$. The temperature response function can be calculated both for the $171 \AA$ channel and the Al-poly of XRT, using the routines of the CHIANTI database (Dere et al. 1997, 2019). After calculating the $\mathrm{X}$-ray and EUV radiation at each grid point, we integrate along the $y$-direction to get a side view. That can be considered as a near-the-limb observation. Similarly, we integrate along the $z$-direction to get a top view. That would correspond to a disk center observation. Both the side and top view as synthesized from our model is shown in Fig. 6.1 for the runs R50, R100, R200. Each plot depicted in Fig. 6.1 is scaled with the maximum value of the EUV or X-ray emission. For the larger numerical boxes, the EUV and X-ray emission increases indicating a connection to the amount of surface magnetic flux each run host at the bottom boundary. As we approach the upper part of the corona, the magnetic field is weak. Hence, we observe a diffused emission. All three runs show bright loops in EUV and X-ray. The difference lies in the length of each loop. The average length of coronal loops in the R200 is on the order of $80 \mathrm{Mm}$ that is significantly larger than the average length of $20 \mathrm{Mm}$ found in R50.

As a next step, we study the temporal variation of the coronal emission. That is an important step since the quantities studied in scaling relations might vary significantly in time, especially during the initial stages of the simulations. Hence, to properly analyze our data, we have to choose an appropriate time frame. The time range where these quantities 

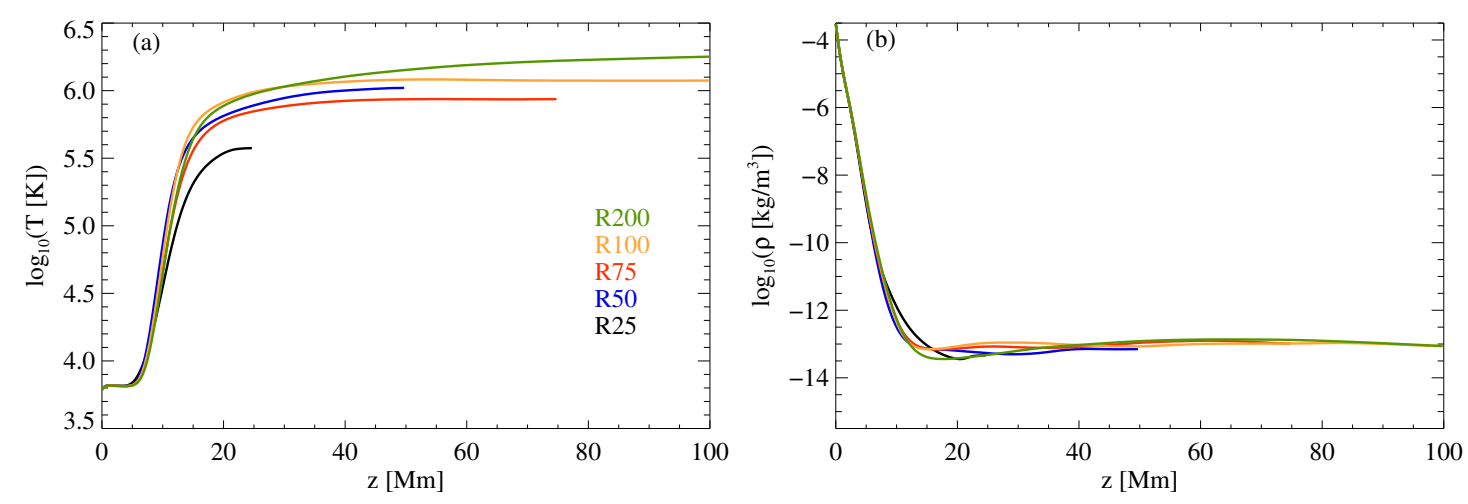

Figure 6.3: Horizontally averaged profile of temperature and density. a) Horizontal average temperature as a function of height for each run. b) Horizontal density as a function of height for each run. The colors show the different runs. Both quantities are also averaged in time over the relaxed phase (see Sect. 6.3.1).

show the least temporal variation, compared to an average value, can be considered as the relaxed phase of our simulations. In Fig. 6.2 it is illustrated the temporal evolution of the coronal X-ray emission and the EUV emission for all the runs listed in Table 6.1. The $\mathrm{X}$-ray and EUV emission in the corona is integrated into the whole computational box. One important aspect we notice here is the connection between magnetic activity and coronal emission. The larger box size, hosting a higher amount of surface magnetic flux, shows a much brighter X-ray and EUV corona than the smaller box sizes. The increase is non-linear, and the implications are discussed in Sect. 6.3.6.

The initial phase determines the cooling phase of our simulations. The duration of that phase depends on the strength of the coronal activity. For the R25 (see black solid line Fig. 6.2) run, since it hosts the least amount of surface magnetic flux, the coronal temperature is too low to sustain a hot corona. Therefore, the cooling time will be much shorter than the other more active runs. The temporal evolution of the coronal emission shows a minimal time variability for the range from $t=220 \mathrm{~min}$ to $t=300 \mathrm{~min}$ indicated by the dashed vertical dashed lines. We consider, for that specific time frame, the simulations to have reached a relaxed phase. The analysis following next will be concentrated at the relaxed phase, hence for the time $220 \mathrm{~min}$ to $300 \mathrm{~min}$.

\subsubsection{Coronal temperature and density}

The temperature and density stratification over height provide insight into the ability of our model to create a hot corona. In this section, we show horizontally averaged profiles of temperature $T$ and density $\rho$. Both quantities are also averaged in time over the relaxed phase. The time interval is chosen appropriately so that the horizontally averaged quantities show minimal variability compared to an average value (see Sect. 6.3.1 and Fig. 6.2). By increasing the size of the box, we allow energy to be deposited in a larger volume. That will lead to an overall increase in the coronal temperature. The average stratification of $T$ and $\rho$ are depicted in Fig. 6.3. Almost all the runs can self-consistently form a hot corona (see Fig. 6.3a). Therefore, the size of the numerical box affects the average coronal temperature. The values for temperature shown in Fig. 6.3a are average values, and thus 
peak temperature in the coronal part of our computational domain is much higher. The smallest box size, R25, does not manage to reach a high coronal temperature. That is to be expected considering the low surface magnetic flux of this specific run. On the other hand, the averaged density $\rho$ (see Fig. 6.3b) shows only a slight increase mainly at the low corona, but there is no clear distinction between the runs as in the temperature profile.

To understand the reasons behind this particular behavior in temperature and density, we have to go back to the RTV scaling laws. As we mentioned in Chap. 2 the RTV scaling laws are an outcome of a 1D hydrostatic coronal loop model. They provide a simple way to relate the coronal temperature and density with the pressure and loop length. Alternatively, it can be written in such a way that to relate the temperature $T$ and density $\rho$ with the heating rate $H$ and the loop length $L$ (see e.g. Zhuleku et al. 2020),

$$
\begin{aligned}
& T \propto H^{2 / 7} L^{4 / 7} \\
& n \propto H^{4 / 7} L^{1 / 7} .
\end{aligned}
$$

Since the strength of the magnetic field is kept constant, so is the amount of energy per unit volume in the corona. In other words, the volumetric heating rate $H$ does not depend on the size of the numerical box, and it will be the same for each run. Consequently, the difference in temperature and density is solely because of the change in the loop length $L$. As a result, if the loop length doubles in size, the temperature increases by a factor of $2^{4 / 7} \simeq 1.5$. On the other hand, the difference in density will only be $2^{1 / 7} \simeq 1$. That explains why the change in density is insignificant for each run.

\subsubsection{Coronal heating}

In our model, the heating is provided by the currents that are induced by the random photospheric motion (see nanoflare model by Parker 1972). That is known as Joule heating. To understand how the Joule heating affects the coronal heating in the current experiments of this chapter, we need to think about how the uniform rescaling of the computational box changes the amount of energy injected in the corona. Since the strength of the vertical photospheric magnetic field is constant, the (volumetric) Ohmic heating rate $H$ should be the same for all the runs listed in Table 6.1. However, the total coronal heating $H_{\text {tot }}^{\text {th }}$ deposited in the corona increases proportionally to the size of the box. Theoretically, this implies that the total coronal heating $H_{\text {tot }}^{\text {th }}$ in each run is related to the total volume $V$,

$$
H_{\text {tot }}^{\text {th }} \propto V
$$

Since all the numerical boxes are chosen to be cubes, that would mean that the volume is $V=D^{3}$. The previous equation can therefore be written as,

$$
H_{\text {tot }}^{\text {th }} \propto D^{3} .
$$

In our case, the numerical boxes are scaled uniformly. That means that the choice of a length scale is not important. We choose the length scale $D$ to be represented by the separation length between the two opposite magnetic polarities of the active region.

As a next step, we investigate the Eq. (6.3) from our numerical runs. For each of the experiments, we determine the separation distance $D$ of the two magnetic polarities, and 

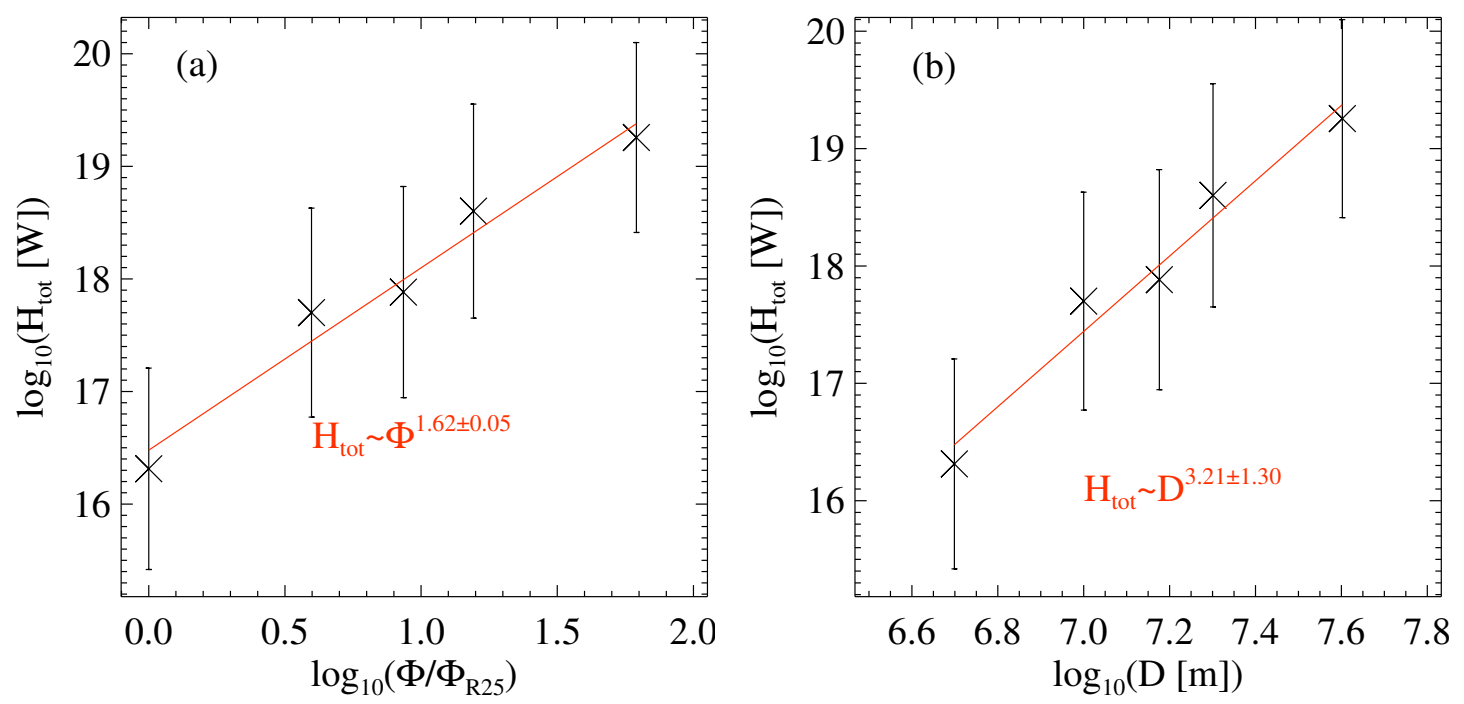

Figure 6.4: Scaling of total coronal heating $\left\langle H_{\text {tot }}\right\rangle$ as a function of the surface magnetic flux $\Phi$ and the separation distance $D$. Each point represents one of the runs with the different spatial size listed in Table 6.1. Quantities are also averaged in time during the relaxed phase of the simulations (see Sect.6.3.1). The bars represent the standard deviation of the total coronal heating $\left\langle H_{\text {tot }}\right\rangle$ in time. Red solid line are power-law fits to the five data points.

we calculate the average total coronal heating $\left\langle H_{\text {tot }}\right\rangle$ inside the coronal volume. The coronal volume is determined as the volume above the height where the horizontally averaged temperature is $0.1 \mathrm{MK}$, similar to what we did in Chap. 5. The $\left\langle H_{\text {tot }}\right\rangle$ is also averaged in time during the relaxed phase. In Fig. 6.4b we depict the scaling plot of the averaged total coronal heating $\left\langle H_{\text {tot }}\right\rangle$ as a function of the magnetic polarity separation distance $D$. Each data point represents an average value for the total coronal heating for each run listed in Table 6.1. A power-law fit to the data yields

$$
\left\langle H_{\text {tot }}\right\rangle \propto D^{q} \quad \text { with } \quad q=3.21 \pm 1.30
$$

Considering the uncertainties, this agrees well with what is expected from Eq. (6.3). In Sect. 6.3.5, we discuss an alternative way to understand Eq. (6.3).

Finally, we relate the total coronal heating to the amount of surface magnetic flux for each experiment. For that, we need to estimate the coronal loop length. The overall coronal loop length is proportional to the distance $D$. From our problem setup, we determine the distance $D$ to be related to the square root of the area $A$,

$$
D \propto A^{1 / 2} .
$$

Substituting this relation to Eq. (6.3) yields,

$$
H_{\text {tot }}^{\text {th }} \propto A^{3 / 2} .
$$

The increase of the surface magnetic flux is proportional to the surface area. Therefore, $\Phi \propto A$, 


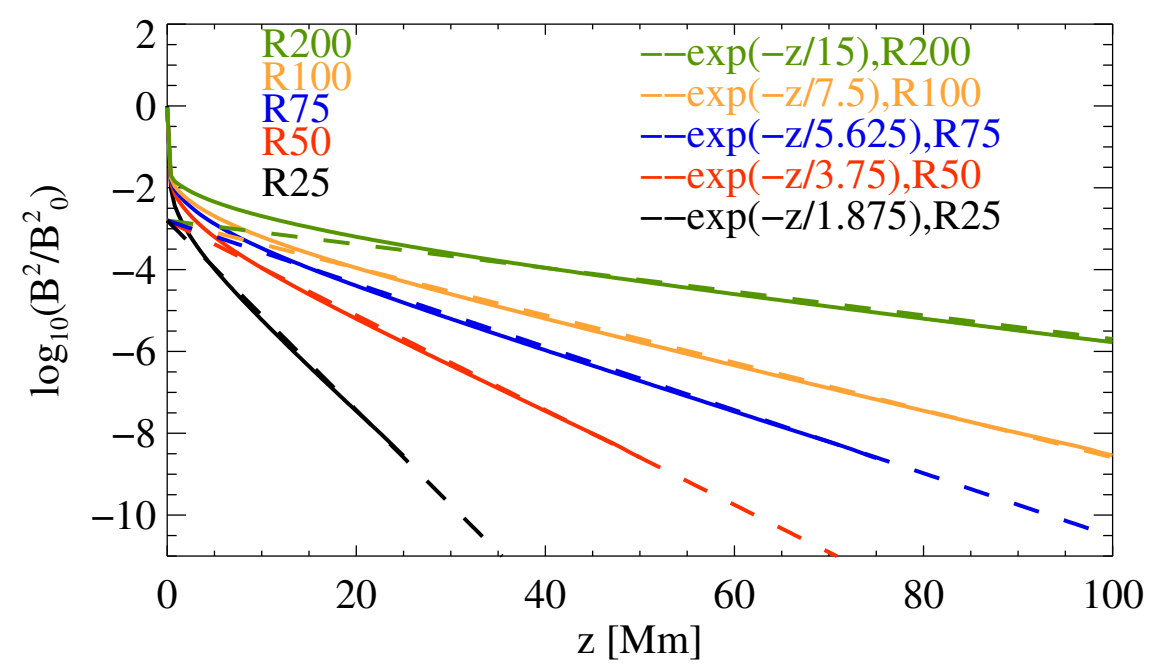

Figure 6.5: Exponential decay of the magnetic field squared $B^{2}$. Solid lines show the horizontal and temporal average profile of $\left\langle B^{2}\right\rangle$ normalised by the value $B_{0}^{2}$ at the surface(i.e. for $z=0$ ). The dashed line show an exponential fit to the $\left\langle B^{2}\right\rangle$ profile with the individual scale heights for each run, listed in Table 6.1, indicated in the legend.

$$
H_{\text {tot }}^{\text {th }} \propto \Phi^{3 / 2}
$$

In Fig. 6.4a we show the scaling relation between the averaged total coronal heating $\left\langle H_{\text {tot }}\right\rangle$ and the surface magnetic flux $\Phi$. A power-law fit to the data points yield,

$$
\left\langle H_{\text {tot }}\right\rangle \propto \Phi^{p} \quad \text { with } \quad p=1.62 \pm 0.05
$$

To conclude, our numerical experiments behave according to what is expected from our simple theoretical estimations from Eq. (6.3) and Eq. (6.7).

\subsubsection{Exponential decay of the magnetic field}

The increase of the coronal magnetic energy can be interpreted as a result of the increase in the separation length between the two polarities of the active region. Once we scale the size of the box, we also increase the length between the two magnetic polarities. The increase of the separation length between the two polarities will change the overall configuration and behavior of the magnetic field.

The determination of the magnetic field poses a serious challenge in our attempt to model the magnetic field distribution in the solar and stellar atmosphere. In general, observations can only provide us with values of the photospheric magnetic field. Based on that we can construct numerical routines to extrapolate the magnetic field in the whole computational domain. For the steady-state case, to determine the magnetic field, we need to find solutions for the two Maxwell equations,

$$
\begin{aligned}
\nabla \times B & =\boldsymbol{j}, \\
\nabla \cdot B & =0 .
\end{aligned}
$$


That is a boundary value problem, but to fully solve the above equations, we also need an additional assumption on the electric currents at the photosphere. This is in general very challenging and most studies use a force-free approximation (i.e. $\boldsymbol{j}=\alpha \boldsymbol{B}$ ) (see e.g. Alissandrakis 1981; Gary 1989). In general, for the coronal plasma to be force-free, the plasma $\beta$ has to be much smaller than unity. However, this is not always the case in the corona (Peter et al. 2015). Finally, the constant $\alpha$ might vary in space and the problem becomes much more complicated (see e.g. Heyvaerts et al. 1979; Sakurai 1979).

In our model, we simplify the process even further. We assume the electric currents to vanish at the photosphere and corona (i.e. $\boldsymbol{j}=0$ ) and seek potential field solutions. We choose the $z$-axis to be perpendicular to the surface plane. The boundary conditions are specified at the solar photosphere for $z=0$. We want to find the magnetic field for $z>0$ extending up to the corona, which solves Eq. (6.9) and also satisfies the given boundary conditions. An easy and fast approach to solve the problem is to work in Fourier space. In this case, Eq. (6.9) following Bracewell (1965) transform to,

$$
\begin{array}{r}
i k_{y} \hat{B}_{z}-\partial_{z} \hat{B}_{y}=0 \\
\partial_{z} \hat{B}_{x}-i k_{x} \hat{B}_{z}=0 \\
i k_{x} \hat{B}_{y}-i k_{y} \hat{B}_{x}=0 \\
k_{x} \hat{B}_{x}+k_{y} \hat{B}_{y}+\partial_{z} \hat{B}_{z}=0 .
\end{array}
$$

Here we consider the $\hat{B}_{x}\left(k_{x}, k_{y}, z\right), \hat{B}_{y}\left(k_{x}, k_{y}, z\right), \hat{B}_{z}\left(k_{x}, k_{y}, z\right)$ to represent the components of the magnetic field in Fourier space. Furthermore, $k_{x}$ and $k_{y}$ are the horizontal wavenumbers. In addition, we assume a priori solutions that satisfy the boundary conditions. The vertical Fourier component of the magnetic field decays exponentially with height,

$$
\hat{B}_{z}\left(k_{x}, k_{y}, z\right)=e^{-k z} \hat{B}_{z}\left(k_{x}, k_{y}, z=0\right) \text {. }
$$

The general solution of Eq. (6.10) can be specified when the determinant of the coefficients vanish. This will determine the value of $k^{2}=k_{x}^{2}+k_{y}^{2}$. To calculate the components of the magnetic field in real space, we use the inverse Fourier transform, which provides our final solutions,

$$
\boldsymbol{B}(x, y, z)=\int_{-\infty}^{\infty} \int_{-\infty}^{\infty} \hat{\boldsymbol{G}}\left(k_{x}, k_{y}, z\right) \hat{B}\left(k_{x}, k_{y}, z=0\right) d k_{x} d k_{y} .
$$

Here $\boldsymbol{G}$ can also be considered to represent the Green function which solves Eq. (6.9). Where the components of the $\hat{\boldsymbol{G}}$ are,

$$
\begin{aligned}
& \hat{G_{x}}=-i \frac{k_{x}}{k} e^{-k z} \\
& \hat{G}_{y}=-i \frac{k_{y}}{k} e^{-k z} \\
& \hat{G}_{z}=e^{-k z}
\end{aligned}
$$

We find in our numerical models that the square of the magnetic field $\boldsymbol{B}^{2}$ in the corona drops as an exponential, 


$$
\boldsymbol{B}^{2}=\boldsymbol{B}_{\mathbf{0}}^{2}(x, y) e^{-k\left(z-z^{\prime}\right)},
$$

where $\boldsymbol{B}_{\mathbf{0}}^{2}(x, y)$ is the magnetic field at height $z=z^{\prime} . z^{\prime}$ denotes the base of the corona, and $k$ is related to the scale height of the exponential drop of the magnetic field. More precisely, $k$ is inversely proportional to the scale height $\lambda$ (i.e. $k=1 / \lambda$ ). That is an outcome of the potential field solutions we have in our model. The scale height $\lambda$ is proportional to the separation length $D$. The wavenumber, as mentioned, is defined as, $k=\sqrt{k_{x}^{2}+k_{y}^{2}}$, where $k=1 / \lambda$ and the horizontal wavenumbers $k_{x}=2 \pi / D$ and $k_{y}=2 \pi / L$. Since the length scales of the numerical box $L$ and the separation distance of the opposite polarities $D$ are proportional to each other, the scale height $\lambda$ is also proportional to $D$ (i.e. $\lambda \propto D$ ). We conclude that the large-scale magnetic field in the corona is close to a potential field. In Fig. 6.5 we show the horizontal average decrease of the magnetic field squared $\boldsymbol{B}^{2}$ for each run. We find that for all runs, the coronal magnetic field drops exponentially. The scale height for each run scales in the same way as the numerical box. If the physical size of the box doubles, the scale height $\lambda$ also doubles.

\subsubsection{Coronal heating problem revisited}

The dissipation of the magnetic energy to thermal is a fundamental process to the coronal heating problem. In Sect. 6.3.3, based on simple power-law estimations, we found that $H_{\text {tot }}^{\text {th }} \propto D^{3}$. Here $H_{\text {tot }}^{\text {th }}$ represents the total coronal heating and $D$ defines the typical length scale in our model. In this section, we revisit the total coronal heating problem based on first principles.

The thermal energy is deposited in the corona through the Ohmic dissipation of the currents. The currents are induced by the random photospheric motion similar to the model of fieldline braiding (Parker 1972). To calculate the total heating deposited in the corona we integrate the Ohmic heating rate $\eta \mu_{0} j^{2}$ inside the coronal volume. The coronal volume is defined in Sect. 6.3.3. We calculate the total coronal heating,

$$
H_{\mathrm{tot}}=\int_{\text {cor }} \eta \mu_{0} \dot{j}^{2} d V=\int_{\text {cor }} \eta \mu_{0} a^{2} \boldsymbol{B}^{2} d V,
$$

where we assumed that in the corona the force free approximation holds therefore $|\boldsymbol{j}|=$ $a|\boldsymbol{B}|$ (see e.g. Gudiksen and Nordlund 2005b). By having an exact analytical function for the magnetic field, the integral in Eq. (6.15) can be calculated analytically. As described in Eq. (6.14), the $\boldsymbol{B}^{2}$ in the coronal part of our computational domain decreases exponentially with a scale height $\lambda$. The scale height $\lambda$ increases as we increase the size of our computational domain. As a result, Eq. (6.15) can be expressed as,

$$
H_{\mathrm{tot}}=\underbrace{\int_{0}^{D} \int_{0}^{D} \eta \mu_{0} a^{2} B_{0}^{2}(x, y) d x d y}_{\propto D^{2}} \underbrace{\int_{z^{\prime}}^{\infty} e^{-\frac{\left(z-z^{\prime}\right)}{\lambda} d z}}_{=\lambda \propto D}
$$

The first double integral represents a surface integral. This double integral is associated with the horizontal average magnetic field at the coronal base (i.e. $z=z^{\prime}$ ). Thus, it will be proportional to the area (or $\propto D^{2}$ ). In addition, the last integral can be solved analytically. 


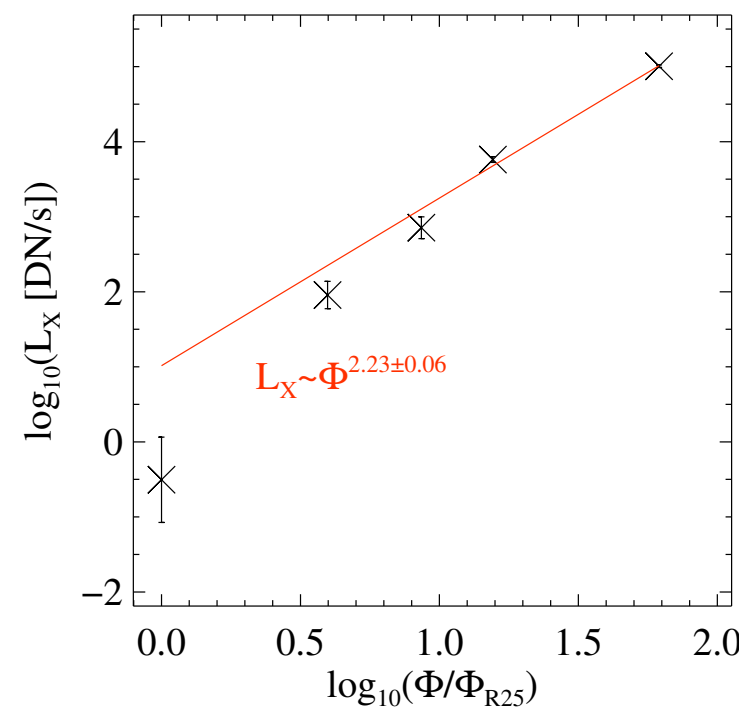

Figure 6.6: Scaling plot of total coronal X-ray emission as a function of unsigned surface magnetic flux $\Phi$ normalised with the reference run R25. Each cross represents on of the numerical runs listed in Table 6.1. The X-ray emission is averaged also in time over the relaxed phase of the simulations (see Sect. 6.3.1). The error bars show the standard deviation in time. Red line show a power-law fit to the data points.

For an upper limit of the integral, we have two choices. The first natural choice is the height of the computational box $L$, where $L$, depending on the model can reach up to $200 \mathrm{Mm}$. However, the bright coronal loops are located at heights that are much smaller compared to the height of the computational box $L$ (see the side view of Fig. 6.1). That leads us to the second choice. We use as an upper limit of the integral to be infinity. As a result, the solution of the last integral is $\lambda$. Since the size of the computational box is scaled uniformly in all directions, then the scale height $\lambda$ is proportional to the polarity separation length $D$. Therefore, the final result is that the total coronal heating reads,

$$
H_{\text {tot }} \propto D^{3} .
$$

Our analytical approach is in good agreement with what we expect based on the simple power-law relations discussed in Sect. 6.3.3 and our numerical models. Overall, we conclude that the coronal magnetic field being close to potential is the fundamental reason why the coronal heating $H_{\text {tot }}$ scales with $D^{3}$.

\subsubsection{Coronal X-ray emission}

The most interesting result of this chapter is the relationship between the total integrated coronal X-ray emission and the surface magnetic flux. That can have an implication to the stellar observations, as discussed in Chap. 5. As the physical size of each box increases, so is the total amount of magnetic energy deposited in the corona. As it is illustrated in the temporal evolution Fig. 6.2, the runs with large active regions exhibit a much higher coronal emission compared to the runs with smaller active regions. That indicates the coronal emission being affected by the amount of surface magnetic flux of each active 
region. To quantify that, we show a scaling plot of the total coronal X-ray emission as a function of the total unsigned surface magnetic flux (see Fig. 6.6). The X-ray emission is integrated into the whole computational domain and averaged in time during the relaxed phase of the simulations. Fig. 6.6 shows a clear non-linear increasing trend of the averaged coronal X-ray emission $\left\langle L_{X}\right\rangle$ for higher surface magnetic flux $\Phi$. A power-law fit to the data yields,

$$
\left\langle L_{\mathrm{X}}\right\rangle \propto \Phi^{m} \quad \text { with } \quad m=2.23 \pm 0.06
$$

To understand the above scaling relations, we develop an analytical expression based on the RTV scaling laws in a similar way as in Zhuleku et al. (2020). The optical thin $\mathrm{X}$-ray radiation $F_{\mathrm{X}}$ is proportional the plasma density squared $n$ times the function $R(T)$,

$$
F_{\mathrm{X}}=n^{2} R(T),
$$

where $F_{\mathrm{X}}$ here has units of energy per volume (i.e. $\left[\mathrm{W} / \mathrm{m}^{3}\right]$ ). The function $R(T)$ is also known as the temperature response function, and it differs from one instrument to another. A detailed explanation can be found in the work of Zhuleku et al. (2020), where it is shown that that the function $R(T)$ for different X-ray instruments can be well approximated as a power-law $R(T) \propto T^{\alpha}$, for temperatures below $\log _{10}(T[K])=7$. Using the well known RTV scaling laws (i.e. $T \propto H^{2 / 7} L^{4 / 7}, n \propto H^{4 / 7} L^{1 / 7}$ ), we can write the X-ray emission $F_{\mathrm{X}}$ as,

$$
F_{\mathrm{X}} \propto L^{p} \quad \text { with } \quad p=2\left(\frac{2 \alpha+1}{7}\right) .
$$

In this case, the heating rate $H$ has no dependence on the surface magnetic flux. Thus, we ignore it in our analysis. Substituting Eq. (6.5) we get,

$$
F_{\mathrm{X}} \propto \Phi^{q} \quad \text { with } \quad q=\frac{2 \alpha+1}{7} .
$$

As a next step, we write the X-ray emission as a function of the surface magnetic flux. To calculate the theoretical X-ray emission, $L_{\mathrm{X}}^{\text {th }}$ of the Sun or a star, it has to be integrated into the whole volume $V=L^{3}$,

$$
L_{\mathrm{X}}^{\mathrm{th}}=F_{\mathrm{X}} V=F_{\mathrm{X}} L^{3} .
$$

Substituting Eq. (6.5) and Eq. (6.21) yields our final result,

$$
L_{\mathrm{X}}^{\text {th }} \propto \Phi^{m^{\prime}} \quad \text { with } \quad m^{\prime}=\frac{4 \alpha+23}{14} .
$$

Interestingly, the result from our analytical approach only depends on the instrument used for the observations. For the XRT instrument onboard Hinode we use $\alpha=2.1$, thus, $m^{\prime}=2.24 \pm 0.75$ which is very close to the value we get from our numerical experiments $m=2.23 \pm 0.06$. The latter one is shown as a red line in Fig. 6.6. 


\subsection{Conclusion}

In this chapter, we performed a number of 3D MHD simulations of active regions. Our goal was to study how the coronal X-ray emission changes with the surface magnetic flux. This relation was found from observations to follow a power-law, $L_{\mathrm{X}} \propto \Phi^{m}$, where the power-law index $m$ is found to be in the range from one to three (Fisher et al. 1998; Pevtsov et al. 2003; Vidotto et al. 2014; Kochukhov et al. 2020). The reasons behind this relationship are still under debate.

To increase the surface unsigned magnetic flux, we increase the surface area covered by the active region while the peak surface magnetic field strength is constant. We start by a small active region inside a computational domain of $25 \times 25 \mathrm{Mm}^{2}$, and we span to a large active region inside a computational domain of 200x200 $\mathrm{Mm}^{2}$. By following this approach, we allow energy to be deposited in a larger coronal volume increasing the X-ray emission. We found the X-ray emission to follow a power-law with a power-law index $m=2.2$.

Based on the RTV scaling laws, we derived an analytic expression of the X-ray emission (see Eq. (6.23)) similar to the approach we follow in Zhuleku et al. (2020). We found that the power-law index $m$ of the X-ray emission will only depend on the instrument used for the X-ray observations. The results of our numerical experiments agree well with our analytical approach. Thus we can explain why the power-law index $m$ is almost quadratic (i.e. $m=2.2$ ).

The linear relation of $L_{X} \propto \Phi$ reported by Fisher et al. (1998) or, Pevtsov et al. (2003) can be explained by an increase of the number of active regions. If a star like our Sun, which has low activity, doubles the number of active regions, then the overall coronal Xray emission and surface magnetic flux are also expected to double. Stars more active than the Sun that have filled their entire surface with magnetic field will not have additional space to fit more active regions. In that case, the peak surface magnetic field strength of each active region has to increase. For this case we showed that steep power-law of $m=3.4$ is expected (see Chap. 5). In the alternative case, considering active regions with a constant surface magnetic field but different surface sizes, we showed that the powerlaw index $m=2.2$ is less steep. This result is closer to the recent studies of Vidotto et al. (2014); Kochukhov et al. (2020) but is a bit steeper than linear that is expected from numerous alternative studies.

In conclusion, a star should not only increase the peak vertical surface magnetic field but also its surface distribution (i.e. more active regions or bigger active regions). This mixture of the two approaches studied in this thesis could explain the overestimation of the power-law index. Overall, the results we find in this chapter and in Chap. 5 can explain the coronal X-ray increase with the surface magnetic flux for stars. Future studies can employ, for example, global scale simulations of an entire surface of a star to fully understand the coronal X-ray emission dependence on the surface magnetic flux. 


\section{Conclusion and Discussion}

In this work, we study the impact of the surface magnetic flux $\Phi$ of the Sun or a star on the coronal X-ray emission $L_{\mathrm{X}}$. Numerous observational studies have found this relationship to follow a power law of $L_{\mathrm{X}} \propto \Phi^{m}$. The power-law index $m$ is found from observations to be in the range from one to almost three. Currently, there is no definite explanation of why the stellar X-ray emission increases as a power-law with the surface magnetic flux and why the observed index $m$ varies. Understanding this relationship is important because it can, for example, explain the mechanism responsible for the heating of the corona of the Sun and the stars. To investigate this relationship, we develop an analytical model based on simple scaling laws, and we perform two parameter studies using 3D MHD simulations of stellar coronae above active regions.

Based on the RTV scaling laws together with the instrumental dependence on temperature, we find an analytical expression for the $L_{\mathrm{X}} \propto \Phi^{m}$. We find the index $m$ to depend on a few key parameters. First of all, it depends on the temperature response of the X-ray instrument used for observations, parameterized by the parameter $\alpha$ (see Chap. 3). Furthermore, the heating mechanism, parameterized by the parameter $\beta$, equally contributes to the index $m$. Finally, there is also a significant contribution from the power-law dependence of the solar or stellar surface area covered by an active region with the surface magnetic flux. This factor is parameterized by the parameter $\delta$. For both heating mechanisms included in our model, i.e. nanoflare $(\beta=2)$ and Alfvén wave $(\beta=1)$, the resulting index $m$ is found to be inside the observational range. However, our model slightly favors the nanoflare mechanism since the upper limit of the index $m$ is found to be closer to more recent studies (Vidotto et al. 2014; Kochukhov et al. 2020). Interestingly, the sensitivity of each X-ray instrument on a specific temperature range is quite significant and can lead to an overall difference in the index $m$ up to $60 \%$.

As a next step, we conducted a series of numerical experiments to study how the coronal X-ray emission is affected by the surface magnetic flux of an active region. We change the surface magnetic flux in our computational box, first, by changing the peak vertical surface magnetic field strength and second, by changing the size of the active region. For both cases, we use the solar coronal model of Bingert (2009) which successfully reproduces many aspects of the solar corona. For our first numerical experiment, we find that the average temperature and density dependence on coronal loop properties are found to agree quite well with what is expected by the RTV scaling laws. In addition, we find that the average Poynting flux at the base of the corona increases almost quadratically with the surface magnetic flux $\left(S_{z} \propto \Phi^{\beta}\right.$ with $\left.\beta=1.71 \pm 0.42\right)$. This is consistent with the fieldline braiding/nanoflare mechanism (Parker 1983) which is considered to be the most probable mechanism responsible for coronal heating. Furthermore, we synthesize the coronal X-ray emission using the temperature response function of the XRT instru- 
ment onboard Hinode. We find a non-linear increase of the $L_{X}$ with a power-law index, $m=3.44 \pm 0.28$. For the second numerical experiments, we find that the magnetic field in the corona is close to a potential field. We report that the magnetic field in the corona decreases exponentially with a specific scale height $\lambda$ for each runs. The scale height $\lambda$ is related to the separation distance between the two polarities of the active region. Therefore, a higher separation distance would make the magnetic field decrease with a larger scale height, allowing higher magnetic energy deposited in the corona. The exponential decay of the magnetic field provides a further explanation of why the total coronal heating is proportional to the volume of the numerical box (see Sect. 6.3.5). Finally, we find the $\mathrm{X}$-ray emission to follow a power-law relation with the surface magnetic flux $L_{\mathrm{X}} \propto \Phi^{m}$ similar to the first numerical experiments. We find the power-law index $m=2.23 \pm 0.06$. An analytic estimation of the power-law index agrees very well with the results we find from our numerical experiments. For both experiments, the index $m$ is found to be larger than unity. Specifically, for the first experiment, we find $m>3$, which is steeper than what is found in observations.

A linear relation of X-ray emission and magnetic flux can be understood by increasing the number of active regions on the surface of a star. If a star with similar activity as the Sun increases the number of active regions, then the overall coronal X-ray emission and the surface magnetic flux are also increased proportionally. For a star more active than the Sun that has covered its entire surface with active regions, the peak surface magnetic field strength per active region has to increase. Consequently, a steeper than unity power-law index is expected. This is consistent with the results of our first numerical experiments. In the case where active regions are larger in size, the power-law index $m$ is found to be larger than unity but consistent with recent observational studies(see Vidotto et al. 2014; Kochukhov et al. 2020). Overall, we understand the difference between our models and observations if a star not only increases the number of active regions but also the peak surface magnetic field strength of each active region. The two experiments presented in this work can explain why the coronal X-ray emission increases as a power-law with the surface magnetic flux for stars.

Currently, all the observational studies, such as, for example, by Pevtsov et al. (2003), combined data from stars with different spectral types and activity levels, which were also observed by different instruments. The sensitivity of each X-ray instrument on the temperature range, as found in our analytical model, is significant. That can potentially explain why different studies observe a different power-law index $m$. A more systematic observational study is required to shed light on this problem.

Estimating the surface magnetic flux for most stars is challenging and the various techniques are limited to a small number of stars. Our model can be used to put constraints on the surface magnetic flux of other stars. By measuring the coronal X-ray emission from a sample of stars, and using the power-law index $m$ as derived from our model, we can potentially estimate the surface magnetic flux $\Phi$ and even compare it with the values obtained by Zeemann Doppler Imaging or Zeemann Broadening techniques.

Certain parameters, however, have been overlooked in our numerical model. First of all, the magnetic helicity at the stellar surface is not included in our model. For most stars, the magnetic helicity is difficult to observe. However, Warnecke and Peter (2019a) based on numerical experiments, found that magnetic helicity can contribute significantly to the increase of the stellar coronal X-ray emission. The magnetic helicity can increase 
the horizontal photospheric magnetic field and thus increase the overall Poynting flux injected from the bottom boundary. This effect is not captured from our model but could potentially affect the power-law indices of $m$ we presented in this work. Another aspect not considered in our model is the quenching of the horizontal motions near the location of a strong magnetic field. This effect can reduce the Poynting flux generated in the photosphere and it can potentially also affect the coronal X-ray emission and thus the power-law index $m$. This is also not known how it operates in other stars but future numerical studies could account for this effect. Finally, stars more active than the Sun are speculated to have stronger photospheric velocities. That is also expected to change the energy input at the photosphere (or the Poynting flux), which will also alter the coronal X-ray emission. However, the photospheric velocity distribution for other stars is observationally ill constraint, therefore in our model is kept at solar values. To conclude, a future study needs to account for all these aspects to investigate the effect on the coronal $\mathrm{X}$-ray emission and the power-law index $m$. That will provide more feedback to our analytical and numerical studies. 



\section{Bibliography}

Abbett, W. P.: 2007, ApJ 665(2), 1469

Alissandrakis, C. E.: 1981, AEA 100(1), 197

Antiochos, S. K., MacNeice, P. J., Spicer, D. S., and Klimchuk, J. A.: 1999, ApJ 512(2), 985

Aschwanden, M. J.: 2005, Physics of the Solar Corona. An Introduction with Problems and Solutions (2nd edition), Springer-Verlag Berlin Heidelberg New York

Aschwanden, M. J., Schrijver, C. J., and Alexand er, D.: 2001, ApJ 550(2), 1036

Benevolenskaya, E. E.: 2007, Advances in Space Research 39(9), 1491

Bingert, S.: 2009, Heating of the corona in a 3D MHD forward model approach, Univ. Freiburg (Breisgau), http://dx.doi.org/10.23689/fidgeo-19

Bingert, S. and Peter, H.: 2011, AEFA 530, A112

Bingert, S. and Peter, H.: 2013a, AEFA 550, A30

Bingert, S. and Peter, H.: 2013b, AE्EA 550, A30

Boris, J. P.: 1970, in A Physically Motivated Solution of the Alfven Problem:NRL memorandum report

Bourdin, P. A., Bingert, S., and Peter, H.: 2013, AE्FA 555, A123

Bourdin, P.-A., Bingert, S., and Peter, H.: 2016, A\&A 589, A86

Bracewell, R.: 1965, The Fourier Transform and its applications, McGraw-Hill, New York

Bradshaw, S. J. and Cargill, P. J.: 2006, AEAA 458(3), 987

Brandenburg, A.: 2018, Pencil Code

Brandenburg, A., Johansen, A., Bourdin, P. A., Dobler, W., Lyra, W., Rheinhardt, M., Bingert, S., Haugen, N. E. L., Mee, A., Gent, F., Babkovskaia, N., Yang, C. C., Heinemann, T., Dintrans, B., Mitra, D., Candelaresi, S., Warnecke, J., Käpylä, P. J., Schreiber, A., Chatterjee, P., Käpylä, M. J., Li, X. Y., Krüger, J., Aarnes, J. R., Sarson, G. R., Oishi, J. S., Schober, J., Plasson, R., Sandin, C., Karchniwy, E., Rodrigues, 
L. F. S., Hubbard, A., Guerrero, G., Snodin, A., Losada, I. R., Pekkilä, J., and Qian, C.: 2020, arXiv e-prints p. arXiv:2009.08231

Cameron, R. H., Jiang, J., Schmitt, D., and Schüssler, M.: 2010, ApJ 719(1), 264

Castellanos Durán, J. S., Lagg, A., Solanki, S. K., and van Noort, M.: 2020, ApJ 895(2), 129

Chatterjee, P.: 2018, Geophys. Astrophys. Fluid Dyn., submitted, arXiv: 1806.08166

Cook, J. W., Cheng, C.-C., Jacobs, V. L., and Antiochos, S. K.: 1989, ApJ 338, 1176

Culhane, J. L.: 1969, MNRAS 144, 375

Dahlburg, R. B., Einaudi, G., Taylor, B. D., Ugarte-Urra, I., Warren, H. P., Rappazzo, A. F., and Velli, M.: 2016, ApJ 817(1), 47

Dahlburg, R. B., Einaudi, G., Ugarte-Urra, I., Rappazzo, A. F., and Velli, M.: 2018, ApJ 868(2), 116

Del Zanna, G. and Mason, H. E.: 2018, Living Reviews in Solar Physics 15, 5

Démoulin, P., van Driel-Gesztelyi, L., Mandrini, C. H., Klimchuk, J. A., and Harra, L.: 2003, ApJ 586(1), 592

Dere, K. P., Del Zanna, G., Young, P. R., Landi, E., and Sutherland, R. S.: 2019, ApJS 241(2), 22

Dere, K. P., Landi, E., Mason, H. E., Monsignori Fossi, B. C., and Young, P. R.: 1997, AEAS 125, 149

Domingo, V., Fleck, B., and Poland, A. I.: 1995, Sol. Phys. 162(1-2), 1

Edlén, B.: 1943, ZAp 22, 30

Feng, C.: 2015, Ph.D. thesis, University of Göttingen

Fisher, G. H., Longcope, D. W., Metcalf, T. R., and Pevtsov, A. A.: 1998, ApJ 508(2), 885

Galsgaard, K. and Nordlund, Å.: 1996, J. Geophys. Res. 101(A6), 13445

Gary, G. A.: 1989, ApJS 69, 323

Getman, K. V., Feigelson, E. D., Micela, G., Jardine, M. M., Gregory, S. G., and Garmire, G. P.: 2008, ApJ 688(1), 437

Giacconi, R., Branduardi, G., Briel, U., Epstein, A., Fabricant, D., Feigelson, E., Forman, W., Gorenstein, P., Grindlay, J., Gursky, H., Harnden, F. R., Henry, J. P., Jones, C., Kellogg, E., Koch, D., Murray, S., Schreier, E., Seward, F., Tananbaum, H., Topka, K., Van Speybroeck, L., Holt, S. S., Becker, R. H., Boldt, E. A., Serlemitsos, P. J., Clark, G., Canizares, C., Markert, T., Novick, R., Helfand, D., and Long, K.: 1979, ApJ 230, 540 
Golub, L., Deluca, E., Austin, G., Bookbinder, J., Caldwell, D., Cheimets, P., Cirtain, J., Cosmo, M., Reid, P., Sette, A., Weber, M., Sakao, T., Kano, R., Shibasaki, K., Hara, H., Tsuneta, S., Kumagai, K., Tamura, T., Shimojo, M., McCracken, J., Carpenter, J., Haight, H., Siler, R., Wright, E., Tucker, J., Rutledge, H., Barbera, M., Peres, G., and Varisco, S.: 2007a, Sol. Phys. 243(1), 63

Golub, L., Deluca, E., Austin, G., Bookbinder, J., Caldwell, D., Cheimets, P., Cirtain, J., Cosmo, M., Reid, P., Sette, A., Weber, M., Sakao, T., Kano, R., Shibasaki, K., Hara, H., Tsuneta, S., Kumagai, K., Tamura, T., Shimojo, M., McCracken, J., Carpenter, J., Haight, H., Siler, R., Wright, E., Tucker, J., Rutledge, H., Barbera, M., Peres, G., and Varisco, S.: 2007b, Sol. Phys. 243(1), 63

Gombosi, T., Tóth, G., Zeeuw, D., Hansen, K., Kabin, K., and Powell, K.: 2002, Journal of Computational Physics 177, 176

Grotrian, W.: 1939, Naturwissenschaften 27(33), 555

Güdel, M.: 2004, AEFA Rev. 12, 71

Gudiksen, B. V., Carlsson, M., Hansteen, V. H., Hayek, W., Leenaarts, J., and MartínezSykora, J.: 2011, AE्FA 531, A154

Gudiksen, B. V. and Nordlund, Å.: 2002, ApJ 572, L113

Gudiksen, B. V. and Nordlund, Å.: 2005a, ApJ 618, 1031

Gudiksen, B. V. and Nordlund, ̊.: 2005b, ApJ 618, 1020

Handy, B. N., Acton, L. W., Kankelborg, C. C., Wolfson, C. J., Akin, D. J., Bruner, M. E., Caravalho, R., Catura, R. C., Chevalier, R., Duncan, D. W., Edwards, C. G., Feinstein, C. N., Freeland, S. L., Friedlaender, F. M., Hoffmann, C. H., Hurlburt, N. E., Jurcevich, B. K., Katz, N. L., Kelly, G. A., Lemen, J. R., Levay, M., Lindgren, R. W., Mathur, D. P., Meyer, S. B., Morrison, S. J., Morrison, M. D., Nightingale, R. W., Pope, T. P., Rehse, R. A., Schrijver, C. J., Shine, R. A., Shing, L., Strong, K. T., Tarbell, T. D., Title, A. M., Torgerson, D. D., Golub, L., Bookbinder, J. A., Caldwell, D., Cheimets, P. N., Davis, W. N., Deluca, E. E., McMullen, R. A., Warren, H. P., Amato, D., Fisher, R., Maldonado, H., and Parkinson, C.: 1999, Sol. Phys. 187(2), 229

Hansteen, V.: 1993, ApJ 402, 741

Hansteen, V., Guerreiro, N., De Pontieu, B., and Carlsson, M.: 2015, ApJ 811(2), 106

Heyvaerts, J., Lasry, J. M., Schatzman, M., and Witomsky, P.: 1979, in E. Jensen, P. Maltby, and F. Q. Orrall (eds.), IAU Colloq. 44: Physics of Solar Prominences, p. 174

Ivanova, N. and Taam, R. E.: 2003, ApJ 599(1), 516

Jansen, F., Lumb, D., Altieri, B., Clavel, J., Ehle, M., Erd, C., Gabriel, C., Guainazzi, M., Gondoin, P., Much, R., Munoz, R., Santos, M., Schartel, N., Texier, D., and Vacanti, G.: 2001, AEFA 365, L1 
Kirichenko, A. S. and Bogachev, S. A.: 2017, Solar Physics 292(9), 120

Klimchuk, J. A.: 2015, Philosophical Transactions of the Royal Society of London Series A 373(2042), 20140256

Kochukhov, O., Hackman, T., Lehtinen, J. J., and Wehrhahn, A.: 2020, AEFA 635, A142

Kosugi, T., Matsuzaki, K., Sakao, T., Shimizu, T., Sone, Y., Tachikawa, S., Hashimoto, T., Minesugi, K., Ohnishi, A., Yamada, T., Tsuneta, S., Hara, H., Ichimoto, K., Suematsu, Y., Shimojo, M., Watanabe, T., Shimada, S., Davis, J. M., Hill, L. D., Owens, J. K., Title, A. M., Culhane, J. L., Harra, L. K., Doschek, G. A., and Golub, L.: 2007, Sol. Phys. 243(1), 3

Landini, M. and Monsignori Fossi, B. C.: 1970, AEFA 6, 468

Lemen, J. R., Title, A. M., Akin, D. J., Boerner, P. F., Chou, C., Drake, J. F., Duncan, D. W., Edwards, C. G., Friedlaender, F. M., Heyman, G. F., Hurlburt, N. E., Katz, N. L., Kushner, G. D., Levay, M., Lindgren, R. W., Mathur, D. P., McFeaters, E. L., Mitchell, S., Rehse, R. A., Schrijver, C. J., Springer, L. A., Stern, R. A., Tarbell, T. D., Wuelser, J.-P., Wolfson, C. J., Yanari, C., Bookbinder, J. A., Cheimets, P. N., Caldwell, D., Deluca, E. E., Gates, R., Golub, L., Park, S., Podgorski, W. A., Bush, R. I., Scherrer, P. H., Gummin, M. A., Smith, P., Auker, G., Jerram, P., Pool, P., Soufli, R., Windt, D. L., Beardsley, S., Clapp, M., Lang, J., and Waltham, N.: 2012, Sol. Phys. 275(1-2), 17

Longcope, D. W., Kankelborg, C. C., Nelson, J. L., and Pevtsov, A. A.: 2001, ApJ 553, 429

MacQueen, R. M., Gosling, J. T., Hildner, E., Munro, R. H., Poland, A. I., and Ross, C. L.: 1974, in D. H. Menzel, L. Larmore, and D. Crawford (eds.), Instrumentation in Astronomy II, Vol. 44 of Society of Photo-Optical Instrumentation Engineers (SPIE) Conference Series, pp 207-212

Magaudda, E., Stelzer, B., Covey, K. R., Raetz, S., Matt, S. P., and Scholz, A.: 2020, $A \mathcal{E} A$ 638, A20

Mandrini, C. H., Démoulin, P., and Klimchuk, J. A.: 2000, ApJ 530(2), 999

Mathioudakis, M., Jess, D. B., and Erdélyi, R.: 2013, Space Sci. Rev. 175(1-4), 1

McIntosh, S. W., de Pontieu, B., Carlsson, M., Hansteen, V., Boerner, P., and Goossens, M.: 2011, Nature 475(7357), 477

Mewe, R. and Gronenschild, E. H. B. M.: 1981, AEFAS 45, 11

Mewe, R., Gronenschild, E. H. B. M., and van den Oord, G. H. J.: 1985, AE्FAS 62, 197

Meyer, J. P.: 1985, ApJS 57, 173

Mok, Y., Mikić, Z., Lionello, R., and Linker, J. A.: 2005, ApJ 621(2), 1098 
Mok, Y., Mikić, Z., Lionello, R., and Linker, J. A.: 2008, ApJ 679(2), L161

Murphy, R. J.: 1985, Ph.D. thesis, University of Maryland

Parker, E. N.: 1972, ApJ 174, 499

Parker, E. N.: 1983, ApJ 264, 642

Parker, E. N.: 1988, ApJ 330, 474

Peres, G., Orlando, S., and Reale, F.: 2004, ApJ 612(1), 472

Peres, G., Orlando, S., Reale, F., and Rosner, R.: 2001, ApJ 563(2), 1045

Pesnell, W. D., Thompson, B. J., and Chamberlin, P. C.: 2012, Sol. Phys. 275(1-2), 3

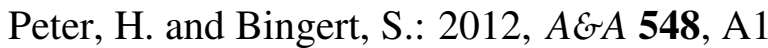

Peter, H., Gudiksen, B. V., and Nordlund, Å.: 2004, ApJ 617, L85

Peter, H., Warnecke, J., Chitta, L. P., and Cameron, R. H.: 2015, AE\&A 584, A68

Pevtsov, A. A., Fisher, G. H., Acton, L. W., Longcope, D. W., Johns-Krull, C. M., Kankelborg, C. C., and Metcalf, T. R.: 2003, ApJ 598(2), 1387

Pfeffermann, E., Briel, U. G., Hippmann, H., Kettenring, G., Metzner, G., Predehl, P., Reger, G., Stephan, K. H., Zombeck, M. V., Chappell, J., and Murray, S. S.: 1986, The Focal Plane Instrumentation Of The ROSAT Telescope, Vol. 733 of Society of PhotoOptical Instrumentation Engineers (SPIE) Conference Series, pp 519-532, SPIE

Pizzolato, N., Maggio, A., Micela, G., Sciortino, S., and Ventura, P.: 2003, AE\&A 397, 147

Priest, E.: 1982, in Solar Magnetohydrodynamics, pp 1-470, Springer Netherlands

Priest, E.: 2014, Magnetohydrodynamics of the Sun, Cambridge University Press

Priest, E. R., Heyvaerts, J. F., and Title, A. M.: 2002, ApJ 576(1), 533

Rappazzo, A. F., Velli, M., Einaudi, G., and Dahlburg, R. B.: 2008, ApJ 677(2), 1348

Reale, F.: 2014, Living Reviews in Solar Physics 11(1), 4

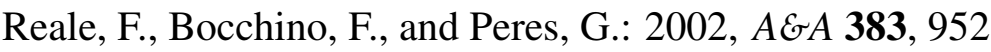

Reale, F., Peres, G., Serio, S., Betta, R. M., DeLuca, E. E., and Golub, L.: 2000, ApJ 535(1), 423

Reale, F., Peres, G., Serio, S., Rosner, R., and Schmitt, J. H. M. M.: 1988, ApJ 328, 256

Reiners, A.: 2012, Living Reviews in Solar Physics 9(1), 1

Reiners, A., Schüssler, M., and Passegger, V. M.: 2014, ApJ 794(2), 144

Rempel, M.: 2017, ApJ 834, 10 
Rosner, R., Tucker, W. H., and Vaiana, G. S.: 1978, ApJ 220, 643

Sakurai, T.: 1979, PASJ 31, 209

Schou, J., Scherrer, P. H., Bush, R. I., Wachter, R., Couvidat, S., Rabello-Soares, M. C., Bogart, R. S., Hoeksema, J. T., Liu, Y., Duvall, T. L., Akin, D. J., Allard, B. A., Miles, J. W., Rairden, R., Shine, R. A., Tarbell, T. D., Title, A. M., Wolfson, C. J., Elmore, D. F., Norton, A. A., and Tomczyk, S.: 2012, Sol. Phys. 275, 229

Schrijver, C. J.: 1983, AEA 127, 289

Schrijver, C. J.: 1987, AEFA 172, 111

Schrijver, C. J., Sandman, A. W., Aschwand en, M. J., and De Rosa, M. L.: 2004, ApJ 615(1), 512

Schrijver, C. J., Title, A. M., Berger, T. E., Fletcher, L., Hurlburt, N. E., Nightingale, R. W., Shine, R. A., Tarbell, T. D., Wolfson, J., Golub, L., Bookbinder, J. A., Deluca, E. E., McMullen, R. A., Warren, H. P., Kankelborg, C. C., Handy, B. N., and de Pontieu, B.: 1999, Sol. Phys. 187(2), 261

Schrijver, C. J., Title, A. M., van Ballegooijen, A. A., Hagenaar, H. J., and Shine, R. A.: 1997, ApJ 487(1), 424

See, V., Matt, S. P., Folsom, C. P., Boro Saikia, S., Donati, J.-F., Fares, R., Finley, A. J., Hébrard, É. M., Jardine, M. M., Jeffers, S. V., Lehmann, L. T., Marsden, S. C., Mengel, M. W., Morin, J., Petit, P., Vidotto, A. A., Waite, I. A., and BCool Collaboration: 2019, ApJ 876(2), 118

Serio, S., Peres, G., Vaiana, G. S., Golub, L., and Rosner, R.: 1981, ApJ 243, 288

Skumanich, A.: 1972, ApJ 171, 565

Solanki, S. K.: 2002, Astronomische Nachrichten 323, 165

Spitzer, L.: 1962, Physics of Fully Ionized Gases, 2nd edition (New York: Interscience)

Tang, F., Howard, R., and Adkins, J. M.: 1984, Sol. Phys. 91(1), 75

Telleschi, A., Güdel, M., Briggs, K., Audard, M., Ness, J.-U., and Skinner, S. L.: 2005, ApJ 622(1), 653

Testa, P., Saar, S. H., and Drake, J. J.: 2015, Philosophical Transactions of the Royal Society of London Series A 373(2042), 20140259

Tomczyk, S., McIntosh, S. W., Keil, S. L., Judge, P. G., Schad, T., Seeley, D. H., and Edmondson, J.: 2007, Science 317(5842), 1192

van Ballegooijen, A. A., Asgari-Targhi, M., Cranmer, S. R., and DeLuca, E. E.: 2011, ApJ 736(1), 3 
van der Holst, B., Sokolov, I. V., Meng, X., Jin, M., Manchester, W. B., I., Tóth, G., and Gombosi, T. I.: 2014, ApJ 782(2), 81

van Wettum, T., Bingert, S., and Peter, H.: 2013, AEA 554, A39

Vernazza, J. E., Avrett, E. H., and Loeser, R.: 1981, ApJS 45, 635

Vidotto, A. A., Gregory, S. G., Jardine, M., Donati, J. F., Petit, P., Morin, J., Folsom, C. P., Bouvier, J., Cameron, A. C., and Hussain, G.: 2014, MNRAS 441(3), 2361

Vögler, A., Shelyag, S., Schüssler, M., Cattaneo, F., Emonet, T., and Linde, T.: 2005, $A \mathcal{E} A$ 429, 335

Warnecke, J. and Bingert, S.: 2020, Geophysical and Astrophysical Fluid Dynamics 114(1-2), 261

Warnecke, J. and Peter, H.: 2019a, AEFA 624, L12

Warnecke, J. and Peter, H.: 2019b, arXiv e-prints p. arXiv:1910.06896

Weisskopf, M. C., Tananbaum, H. D., Van Speybroeck, L. P., and O'Dell, S. L.: 2000, Chandra X-ray Observatory (CXO): overview, Vol. 4012 of Society of Photo-Optical Instrumentation Engineers (SPIE) Conference Series, pp 2-16, SPIE

Withbroe, G. L. and Noyes, R. W.: 1977, ARAEFA 15, 363

Wolfson, R., Roald, C. B., Sturrock, P. A., and Weber, M. A.: 2000, ApJ 539, 995

Wright, N. J. and Drake, J. J.: 2016, Nature 535(7613), 526

Wright, N. J., Drake, J. J., Mamajek, E. E., and Henry, G. W.: 2011, ApJ 743(1), 48

Zhuleku, J., Warnecke, J., and Peter, H.: 2020, AEFA 640, A119 



\section{Scientific contributions}

\section{Refereed publications}

- J. Zhuleku, J. Warnecke and H. Peter, Stellar coronal X-ray emission and surface magnetic flux, 2020, A\&A, 640, A119

\section{Submitted publications}

- J. Zhuleku, J. Warnecke and H. Peter, Stellar X-rays and magnetic activity in 3D MHD coronal models, 2020, submitted in A\&A

\section{Conference contributed talks}

- Hellenic Astronomical Society conference, Volos, Greece, 2-7 July, 2019

- Coronal loop 9 workshop, St. Andrews, Scotland, 11-14 June, 2019

\section{Conference posters}

- Hinode 12 conference, Granada, Spain, 9-15 September, 2018

- IRIS 9 meeting, Göttingen, Germany, 25-29 June, 2018 



\section{Acknowledgements}

First of all, I want to sincerely thank my supervisor, Hardi Peter, for giving me a chance to work with him and for his support and advice. He was an excellent supervisor and I learned many things in our countless meetings. Furthermore, I want to equally thank Jörn Warnecke for his help and support all this years, and that he was always there answering all my "stupid" questions. I also want to thank the rest of the coronal group, Tanayveer Bhatia, Pradeep L. Chitta, Jamie Gorman, and Nikolina Milanovic.

I would also like to thank the International Max Planck Research School at the University of Göttingen and the Max Planck Institute for Solar System Research for funding my project. A sincere thanks also go to Dr. Sonja Schuh for guiding me through all the necessary regulations and requirements during my $\mathrm{Ph} . \mathrm{D}$. studies.

The simulations performed in this thesis have been carried out at the GWDG facilities in Göttingen and the Max Planck Computing and Data Facility (MPCDF) in Garching, München.

Furthermore, I would like to thank Christian Baumgartner and Valentin Bickel for all the emotional support all these years and the many amazing meetings we had in Thanners and Fiz every Saturday. Furthermore, a great appreciation and sincere thanks to Nina Nemec with whom we shared many "funny" and serious discussions during our daily coffee meetings at 15:00. She was always there whenever I had a problem. A big thank you also goes to the good friend Cosima Breu with whom we shared an office, and she had to put up with my presence every day. Finally, I also want to thank all the IMPRS students and postdocs for making our every days more lively.

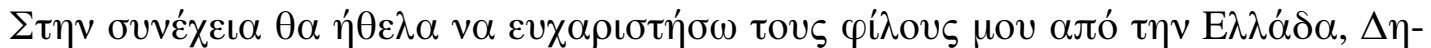

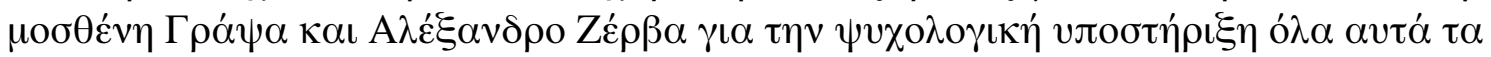

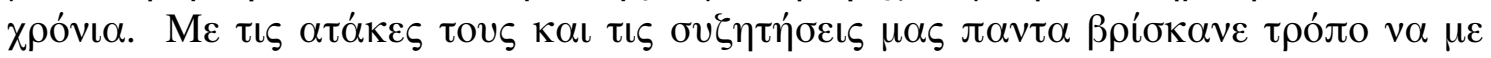

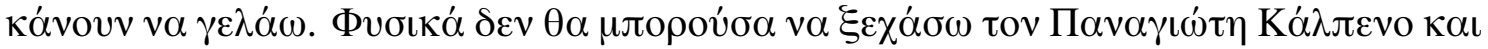

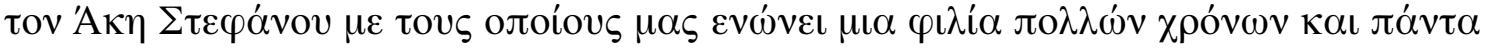

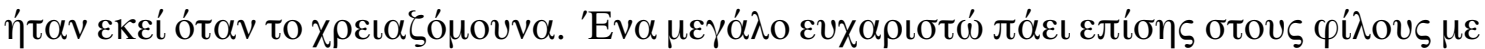

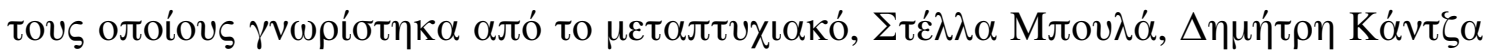

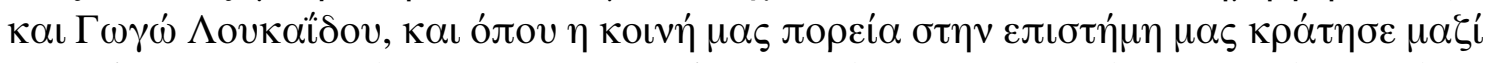

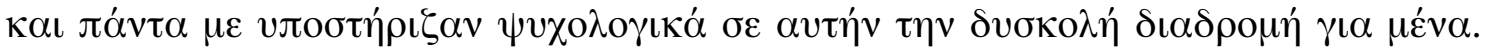

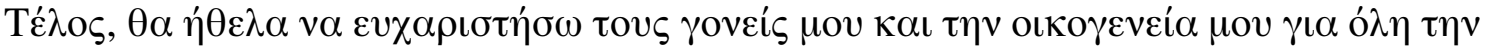

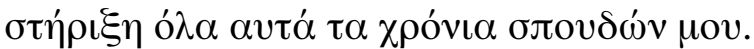

